\title{
The Kepler characterization of the variability among A- and F-type stars
}

\section{General overview}

\author{
K. Uytterhoeven ${ }^{1,2,3}$, A. Moya ${ }^{4}$, A. Grigahcène ${ }^{5}$, J. A. Guzik ${ }^{6}$, J. Gutiérrez-Soto ${ }^{7,8,9}$, B. Smalley ${ }^{10}$, G. Handler ${ }^{11,12}$, \\ L. A. Balona ${ }^{13}$, E. Niemczura ${ }^{14}$, L. Fox Machado ${ }^{15}$, S. Benatti ${ }^{16,17}$, E. Chapellier ${ }^{18}$, A. Tkachenko ${ }^{19}$, R. Szabó ${ }^{20}$, \\ J. C. Suárez ${ }^{7}$, V. Ripepi ${ }^{21}$, J. Pascual ${ }^{7}$, P. Mathias ${ }^{22}$, S. Martín-Ruíz ${ }^{7}$, H. Lehmann²3, J. Jackiewicz ${ }^{24}$, S. Hekker $^{25,26}$, \\ M. Gruberbauer ${ }^{27,11}$, R. A. García ${ }^{1}$, X. Dumusque ${ }^{5,28}$, D. Díaz-Fraile ${ }^{7}$, P. Bradley ${ }^{29}$, V. Antoci ${ }^{11}$, M. Roth $^{2}$, B. Leroy ${ }^{8}$, \\ S. J. Murphy ${ }^{30}$, P. De Cat ${ }^{31}$, J. Cuypers ${ }^{31}$, H. Kjeldsen ${ }^{32}$, J. Christensen-Dalsgaard ${ }^{32}$, M. Breger ${ }^{11,33}$, A. Pigulski ${ }^{14}$, \\ L. L. Kiss ${ }^{20,34}$, M. Still ${ }^{35}$, S. E. Thompson ${ }^{36}$, and J. Van Cleve ${ }^{36}$
}

(Affiliations can be found after the references)

Received 30 May 2011 / Accepted 29 June 2011

\begin{abstract}
Context. The Kepler spacecraft is providing time series of photometric data with micromagnitude precision for hundreds of A-F type stars. Aims. We present a first general characterization of the pulsational behaviour of A-F type stars as observed in the Kepler light curves of a sample of 750 candidate A-F type stars, and observationally investigate the relation between $\gamma$ Doradus ( $\gamma$ Dor), $\delta$ Scuti $(\delta$ Sct), and hybrid stars.

Methods. We compile a database of physical parameters for the sample stars from the literature and new ground-based observations. We analyse the Kepler light curve of each star and extract the pulsational frequencies using different frequency analysis methods. We construct two new observables, "energy" and "efficiency", related to the driving energy of the pulsation mode and the convective efficiency of the outer convective zone, respectively.

Results. We propose three main groups to describe the observed variety in pulsating A-F type stars: $\gamma$ Dor, $\delta$ Sct, and hybrid stars. We assign $63 \%$ of our sample to one of the three groups, and identify the remaining part as rotationally modulated/active stars, binaries, stars of different spectral type, or stars that show no clear periodic variability. $23 \%$ of the stars (171 stars) are hybrid stars, which is a much higher fraction than what has been observed before. We characterize for the first time a large number of A-F type stars (475 stars) in terms of number of detected frequencies, frequency range, and typical pulsation amplitudes. The majority of hybrid stars show frequencies with all kinds of periodicities within the $\gamma$ Dor and $\delta$ Sct range, also between 5 and $10 \mathrm{~d}^{-1}$, which is a challenge for the current models. We find indications for the existence of $\delta$ Sct and $\gamma$ Dor stars beyond the edges of the current observational instability strips. The hybrid stars occupy the entire region within the $\delta$ Sct and $\gamma$ Dor instability strips and beyond. Non-variable stars seem to exist within the instability strips. The location of $\gamma$ Dor and $\delta$ Sct classes in the $\left(T_{\text {eff }}, \log g\right)$-diagram has been extended. We investigate two newly constructed variables, "efficiency" and "energy", as a means to explore the relation between $\gamma$ Dor and $\delta$ Sct stars.

Conclusions. Our results suggest a revision of the current observational instability strips of $\delta$ Sct and $\gamma$ Dor stars and imply an investigation of pulsation mechanisms to supplement the $\kappa$ mechanism and convective blocking effect to drive hybrid pulsations. Accurate physical parameters for all stars are needed to confirm these findings.
\end{abstract}

Key words. stars: oscillations - stars: fundamental parameters - binaries: general - asteroseismology - stars: variables: $\delta$ Scuti - stars: statistics

\section{Introduction}

With the advent of the asteroseismic space missions MOST (Walker et al. 2003), CoRoT (Baglin et al. 2006), and Kepler (Borucki et al. 2010), a new window is opening towards the understanding of the seismic behaviour of A- and F-type pulsators. The main advantages of these space missions are (1) the longterm continuous monitoring of thousands of stars, which enables both the determination of long-period oscillations and the resolving of beat frequencies; and (2) the photometric precision at the level of milli- to micro-magnitudes, which will provide a more complete frequency spectrum and also allow the detection of low-amplitude variations that are unobservable from the ground and providing a more complete frequency spectrum. The availability of these long-term, very precise light curves makes possible the first comprehensive analysis of the variability of a sample of several hundred candidate A-F type stars that is presented here.
The region of variable A- and F-type, including main sequence (MS), pre-MS, and post-MS stars, with masses between 1.2 and $2.5 M_{\odot}$ hosts the $\gamma$ Doradus ( $\gamma$ Dor) and $\delta$ Scuti $(\delta \mathrm{Sct})$ pulsators. The $\gamma$ Dor stars were recognized as a new class of pulsating stars less than 20 years ago (Balona et al. 1994). Our current understanding is that they pulsate in high-order gravity (g) modes (Kaye et al. 1999a), excited by a flux modulation mechanism induced by the upper convective layer (Guzik et al. 2000; Dupret et al. 2004; Grigahcène 2005). Typical $\gamma$ Dor periods are between $8 \mathrm{~h}$ and $3 \mathrm{~d}$. From the ground, about 70 bona fide and 88 candidate $\gamma$ Dor pulsators have been detected (Balona et al. 1994; Handler 1999; Henry et al. 2005; De Cat et al. 2006; Henry et al. 2011, among other papers).

The $\delta$ Sct variables, on the other hand, have been known for decades. They show low-order $g$ and pressure (p) modes with periods between $15 \mathrm{~min}$ and $5 \mathrm{~h}$ that are self-excited through the $\kappa$-mechanism (see reviews by Breger 2000; Handler 2009a). 
Several hundreds of $\delta$ Sct stars have been observed from the ground (e.g. catalogue by Rodríguez \& Breger 2001).

Because the instability strips of both classes overlap, the existence of hybrid stars, i.e. stars showing pulsations excited by different excitation mechanisms, is expected, and a few candidate hybrid stars have indeed been detected from the ground (Henry \& Fekel 2005; Uytterhoeven et al. 2008; Handler 2009b).

The main open question in seismic studies of A- and F-type stars concerns the excitation and mode selection mechanism of $p$ and g modes. The only way to understand and find out systematics in the mode-selection mechanism is a determination of pulsation frequencies and pulsation mode parameters for a large number of individual class members for each of the pulsation classes, and a comparison of the properties of the different case-studies. So far, a systematic study of a sufficiently substantial sample was hampered by two factors. First, the number of detected well-defined pulsation modes is too small to construct unique seismic models, which is caused by ground-based observational constraints, such as bad time-sampling and a high noiselevel. Second, only a small number of well-studied cases exist, because a proper seismic study requires a long-term project, involving ground-based multi-site campaigns spanning several seasons, or a dedicated space mission.

First demonstrations of the strength and innovative character of space data with respect to seismic studies of A-F type stars are the detection of two hybrid $\gamma$ Dor- $\delta$ Sct stars by the MOST satellite (HD 114839, King et al. 2006; BD+18-4914, Rowe et al. 2006), and the detection of an impressive number of frequencies at low amplitudes, including high-degree modes as confirmed by ground-based spectroscopy, in the precise space CoRoT photometry of the $\delta$ Sct stars HD 50844 (Poretti et al. 2009) and HD 174936 (García Hernández et al. 2009), and the $\gamma$ Dor star HD 49434 (Chapellier et al. 2011). The first indications that hybrid behaviour might be common in A-F type stars were found from a pilot study of a larger sample of Kepler and CoRoT stars (Grigahcène et al. 2010; Hareter et al. 2010). Recently, Balona et al. (2011a) announced the detection of $\delta$ Sct and $\gamma$ Dor type pulsations in the Kepler light curves of Ap stars. Hence, a breakthrough is expected in a currently poorly-understood field of seismic studies of A-F type pulsators through a systematic and careful investigation of the pulsational behaviour in a large sample of stars.

The goals of the current paper are (1) to present a first general characterization of the pulsational behaviour of main-sequence A-F type stars as observed in the Kepler light curves of a large sample; and (2) to observationally investigate the relation between $\gamma$ Dor and $\delta$ Sct stars and the role of hybrids. In forthcoming papers, detailed seismic studies and modelling of selected stars will be presented.

\section{The Kepler sample of A-F type stars}

\subsection{The Kepler data}

The NASA space mission Kepler was launched in March 2009 and is designed to search for Earth-size planets in the extended solar neighbourhood (Borucki et al. 2010; Koch et al. 2010). To this end, the spacecraft continuously monitors the brightness of $\sim 150000$ stars in a fixed area of $105 \mathrm{deg}^{2}$ in the constellations Cygnus, Lyra, and Draco, at Galactic latitudes from 6 to $20 \mathrm{deg}$. The nearly uninterrupted time series with micromagnitude precision also opens up opportunities for detailed and in-depth asteroseismic studies with unprecedented precision (Gilliland et al. 2010a). Of all Kepler targets, more than 5000 stars have been selected as potential targets for seismic studies by the Kepler Asteroseismic Science Consortium, KASC ${ }^{1}$.

The Kepler Mission offers two observing modes: long cadence (LC) and short cadence (SC). The former monitors selected stars with a time resolution of $\sim 30$ min (Jenkins et al. 2010a), the latter provides a 1-min sampling (Gilliland et al. 2010b). The LC data are well-suited to search for long-period g-mode variations in A-F type stars (periods from a few hours to a few days), while the SC data are needed to unravel the p-mode oscillations (periods of the order of minutes to hours).

The Kepler asteroseismic data are made available to the KASC quarterly. In this paper we consider data from the first year of Kepler operations: the 9.7 d Q0 commissioning period (1-11 May 2009), the $33.5 \mathrm{~d}$ Q1 phase data (12 May-14 June 2009), the 88.9 d Q2 phase data (19 June-15 September 2009), the 89.3 d time string of Q3 (18 September-16 December 2009), and 89.8 d of Q4 data (19 December 2009-19 March 2010). The SC data are subdivided into three-monthly cycles, labelled, for example, Q3.1, Q3.2 and Q3.3.

Not all quarters Q0-Q4 are available for all stars. The first year of Kepler operations was dedicated to the survey phase of the mission. During this phase as many different stars as possible were monitored with the aim to identify the best potential candidates for seismic studies. From the survey sample, the KASC working groups selected subsamples of the best seismic candidates for long-term follow-up with Kepler. From quarter Q5 onwards, only a limited number of selected KASC stars are being observed with Kepler. The results of the selection process of the most promising $\gamma$ Dor, $\delta$ Sct, and hybrid candidates are presented in this work.

\subsection{Selection of the A-F type star sample}

We selected all stars in the Kepler Asteroseismic Science Operations Center (KASOC) database initially labelled as $\gamma$ Dor or $\delta$ Sct candidates. The stars were sorted into these KASOC catagories either because the Kepler Input Catalogue (KIC; Latham et al. 2005; Brown et al. 2011) value of their effective temperature $T_{\text {eff }}$ and gravity $\log g$ suggested that they lie in or close to the instability strips of $\gamma$ Dor and $\delta$ Sct stars, or because they where proposed as potential variable A-F type candidates in pre-launch asteroseismic Kepler observing proposals. To avoid sampling bias and to aim at completeness of the sample, we analysed all stars listed in the KASOC catalogue as $\delta$ Sct or $\gamma$ Dor candidates. Our analysis results provide feedback on the initial guess on variable class assigment by KASOC. As will be seen (Sect. 6.2), several of these stars actually belong to other pulsation classes, many of which are cool stars. Because there are much fewer B-type stars in the Kepler field of view than cooler stars, there is a natural selection effect towards cooler stars. We also included stars initially assigned to other pulsation types that showed periodicities typical for $\delta$ Sct and/or $\gamma$ Dor stars. We are aware that many more $\delta$ Sct and $\gamma$ Dor candidate stars are being discovered among the KASC targets, but we cannot include all in this study.

The total sample we considered consists of 750 stars. For 517 stars both LC and SC data are available, while 65 and 168 stars were only observed in SC and LC mode, respectively. An overview of the A-F type star sample is given in Table 1, available in the on-line version of the paper. The first three columns indicate the KIC identifier of the star (KIC ID),

\footnotetext{
${ }^{1}$ http://astro.phys.au.dk/KASC
} 
right ascension (RA), declination (Dec), and Kepler magnitude $(\mathrm{Kp})$. The Kepler bandpass is wider than the typical broad-band filters that are commonly used in optical astronomy (e.g. Johnson $U B V R I$ ), and can be described as "white" light. The next three columns provide information on the spectral type (Spectral Type), alternative name of the target (Name), and a comment on its variability (Variable). Information on binarity comes from the Washington Double Star Catalog (Worley \& Douglass 1997; Mason et al. 2001), unless mentioned otherwise. For binary stars labelled with “ $\star$ ”, the double star was suspected by inspecting Digitized Sky Survey and 2MASS images by eye. The next set of columns provides information on the Kepler time series. For each star, the number of datapoints ( $N$ datapoints), the total time span of the dataset $(\Delta T)$ expressed in d, the longest time gap in the Kepler light curves $(\delta T)$ expressed in d, and the available (range of) quarters in LC (Quarters LC) and SC (Quarters SC) mode are given.

\subsection{Sample stars in the literature}

Most of the 750 sample stars were previously unstudied. We searched the catalogue by Skiff (2007) and found information on spectral types for only 212 stars. Besides 198 confirmed Aor F-type stars, among which are fourteen chemically peculiar stars, we discovered that stars with a different spectral type also ended up in the sample. There are six known B stars, one M star, three $\mathrm{K}$ stars, and six G-type stars in the sample. The $\mathrm{G}$ star KIC 7548061 (V1154 Cyg) is a known and well-studied Cepheid (e.g. Pigulski et al. 2009) and is the subject of a dedicated paper based on Kepler data by Szabó et al. (2011). Sixty-two stars are known to belong to multiple systems, including at least fourteen eclipsing binaries (EB; KIC 1432149, Hartman et al. 2004; KIC 10206340, Malkov et al. 2006; catalogues by Prša et al. 2011; and Slawson et al. 2011). Seven stars are only known as "(pulsating) variable stars". The star KIC 2987660 (HD 182634) is reported as a $\delta$ Sct star by Henry et al. (2001). Our sample also includes a candidate $\alpha^{2}$ Canum Venaticorum star, namely KIC 9851142 or V2094 Cyg (Carrier et al. 2002; Otero 2007). The Kepler field hosts four open clusters. In our sample at least six known members of NGC 6819 are included. Also one, eight, and nine members of NGC 6791, NGC 6811, and NGC 6866, respectively, are in our sample. All 750 stars are included in the analysis.

\section{Physical parameters of the sample stars}

Seismic models require accurate values of physical parameters such as $\log g, T_{\text {eff }}$, metallicity $[M / H]$, and projected rotational velocity $v \sin i$. We compiled an overview of all $T_{\text {eff }}, \log g$, and $v \sin i$ values available for the sample stars in Table 2 in the on-line version of the paper. The different sources include literature and KIC, along with values derived from new groundbased data. A description of the different sources is given below. The columns of Table 2 are (1) KIC identifier (KIC ID); (2) $T_{\text {eff }}$ value from KIC; (3)-(5) $T_{\text {eff }}$ values taken from the literature or derived from new ground-based data (Literature); (6) adopted $T_{\text {eff }}$ value (Adopted); (7) $\log g$ value from KIC; (8)-(9) $\log g$ values taken from the literature or derived from new ground-based data (Literature); (10) adopted $\log g$ value (Adopted); (11)-(12) $v \sin i$ values derived from spectroscopic data (Spectra). Stars that are known to be spectroscopic binaries are flagged ${ }^{\circ}$ behind its KIC identifier (KIC ID). The derived physical parameters of the binary stars have to be considered

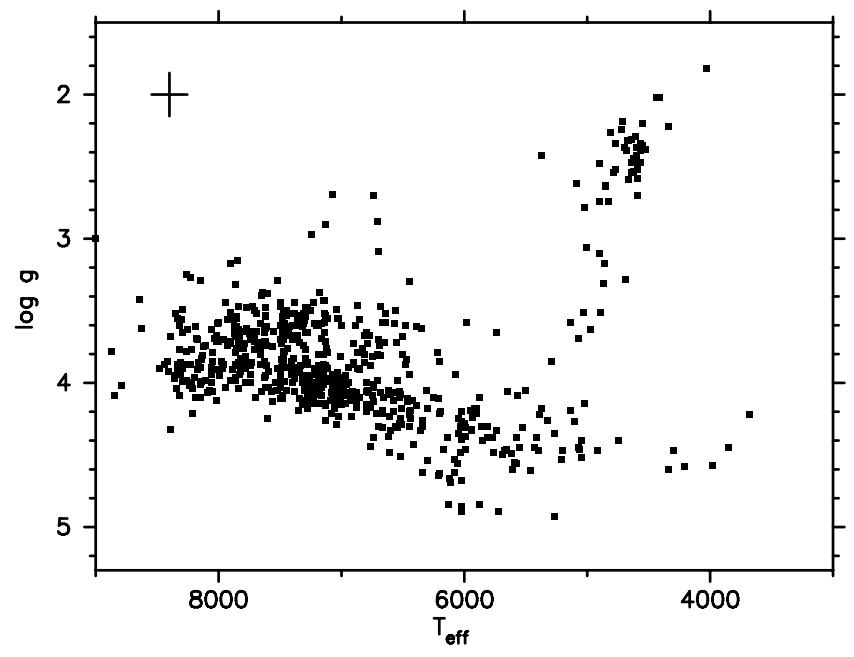

Fig. 1. 750 sample stars in the $\left(T_{\text {eff }}, \log g\right)$-diagram. The cross at the left top corner represents the typical error bars on the values: $290 \mathrm{~K}$ for $T_{\text {eff }}$ and 0.3 dex for $\log g$.

with caution because the contribution of the binary components might not have been correctly separated.

KIC-independent values of $\log g$ and $T_{\text {eff }}$ are only available for 110 stars. The values used for the subsequent analysis are (in order of priority, depending on availability and accuracy) the spectroscopically derived values, or the most recent photometrically derived values. For all other stars we use the only source: the KIC values. The corresponding adopted $T_{\text {eff }}$ (in $\mathrm{K}$ ), and $\log g$ (in dex) values are given in boldface in the sixth and tenth column of Table 2, respectively (column "Adopted"). For 65 and 71 stars no value of $T_{\text {eff }}$ and $\log g$, respectively, is available. Figure 1 shows the sample of 750 stars in the $\left(T_{\text {eff }}, \log g\right.$ )-diagram. We estimated the error bars on the KIC values by comparing them with the adopted values taken from the literature or ground-based data. The average difference was $290 \mathrm{~K}$ for $T_{\text {eff }}$ and 0.3 dex for $\log g . v \sin i$ values are only available for 52 of the sample stars.

\subsection{Literature}

Besides papers dedicated to specific targets of our sample, the on-line catalogues by Soubiran et al. (2010), Lafrasse et al. (2010), Kharchenko et al. (2009), Masana et al. (2006), Nordström et al. (2004), Glebocki \& Stawikowski (2000), Allende Prieto \& Lambert (1999), and Wright et al. (2003) were very helpful in the search for values of $T_{\text {eff }}, \log g$, and $v \sin i$. Also, photometric indices by Hauck \& Mermilliod (1998) were used to estimate values of $T_{\text {eff }}$ and $\log g$. We took care not to include $T_{\text {eff }}$ values that are derived from the spectral type rather than directly from data. The literature values of $T_{\text {eff }}$ and $\log g$ can be found in Cols. 3-5 and 8, 9 of Table 2, respectively ("lit"). We note that the given errors on $T_{\text {eff }}$ and $\log g$, which sometimes seem unrealistic small, are taken from the quoted paper and are not rounded to the number of significant digits. Values of $v \sin i$, expressed in $\mathrm{km} \mathrm{s}^{-1}$, are given in the last two columns of Table 2 . The source of each value is indicated by the label.

\subsection{Kepler Input Catalogue}

The KIC provides an estimate of $T_{\text {eff }}$ and $\log g$ for most Kepler targets derived from Sloan photometry (see the second and 
seventh column, "KIC", of Table 2, respectively). Unfortunately, the KIC values of $\log g$ are known to have large error bars (Molenda-Żakowicz et al. 2011; Lehmann et al. 2011). Moreover, a comparison between KIC estimates of the stellar radius and the radius derived from evolutionary models indicate that the KIC values of $\log g$ might be shifted towards lower values by about 0.1 dex. The temperature values, on the other hand, are fairly good for A-F type stars, and become less reliable for more massive or peculiar stars, because for higher temperatures the interstellar reddening is apparently not properly taken into account. The stars in our sample are reddened up to $0.3 \mathrm{mag}$ in $(B-V)$, with an average reddening of $E(B-V)=0.04$ mag. The 85 stars for which no KIC $T_{\text {eff }}$ value is available, which are generally faint stars $(\mathrm{Kp}>11 \mathrm{mag})$, are not considered in any analysis related to temperature, unless values of $T_{\text {eff }}$ exist in the literature or are available from the analysis of new ground-based observations (see below).

\subsection{New ground-based observations of sample stars}

In the framework of the ground-based observational project for the characterization of KASC targets (see Uytterhoeven et al. 2010a,b, for an overview), targets of the A-F type sample are being observed using multi-colour photometry and/or high-tomid-resolution spectroscopy. The goal is to obtain precise values of physical parameters that are needed for the seismic modelling of the stars. A detailed analysis of a first subsample of A-F type stars has been presented by Catanzaro et al. (2011). Several other papers are in preparation. We include the available results to date in this paper, because the precise values of $T_{\text {eff }}$ and $\log g$ are needed for the interpretations in Sects. 7 and 8.

\subsubsection{Strömgren photometry from the Observatorio San Pedro Mártir}

Multi-colour observations were obtained for 48 sample stars over the period 2010 June 13-17 with the six-channel $u v b y-\beta$ Strömgren spectrophotometer attached to the $1.5-\mathrm{m}$ telescope at the Observatorio Astrónomico Nacional-San Pedro Mártir (OAN-SPM), Baja California, Mexico. Each night, a set of standard stars was observed to transform instrumental observations into the standard system using the well known transformation relations given by Strömgren (1966), and to correct for atmospheric extinction. Next, the photometric data were dereddeded using Moon's UVBYBETA programme (Moon 1985), and $T_{\text {eff }}$ and $\log g$ values were obtained using the $u v b y$ grid presented by Smalley \& Kupka (1997). A detailed description of the data will be given by Fox Machado et al. (in prep.). The resulting stellar atmospheric parameters are presented in Table 2 under label " $b$ ".

\subsubsection{SOPHIE spectra from the Observatoire de Haute Provence}

We also analysed spectra of two sample stars, KIC 11253226 and KIC 11447883, obtained during the nights of 2009 July 31, August 1, and August 5 with the high-resolution $(R \sim 70000)$ spectrograph SOPHIE, which is attached to the 1.93-m telescope at the Observatoire de Haute Provence (OHP), France. The spectra were reduced using a software package directly adapted from HARPS, subsequently corrected to the heliocentric frame, and manually normalized by fitting a cubic spline.
To derive stellar atmospheric parameters, the observed spectra, which covers the wavelength range 3870-6940 $\AA$, were compared with synthetic spectra. The synthetic spectra were computed with the SYNTHE code (Kurucz 1993), using atmospheric models computed with the line-blanketed LTE ATLAS9 code (Kurucz 1993). The parameters were derived using the methodology presented in Niemczura et al. (2009) which relies on an efficient spectral synthesis based on a least-squares optimisation algorithm. The resulting values of $T_{\mathrm{eff}}, \log g$ and $v \sin i$ are presented in Table 2, under label " $h$ ". The detailed analysis results, including element abundances and microturbulence, will be presented in a dedicated paper (Niemczura et al., in prep.), including several other Kepler stars.

\subsubsection{Spectra from the Tautenburg Observatory}

Spectra of 26 sample stars were obtained from May to August 2010 with the Coude-Échelle spectrograph attached to the 2-m telescope of the Thüringer Landessternwarte Tautenburg (TLS), Germany. The spectra cover 4700 to $7400 \AA$ in wavelength range, with a resolution of $R=32000$. The spectra were reduced using standard ESO-MIDAS packages. We obtained between two and seven spectra per star, which were radial velocity corrected and co-added. The resulting signal-to-noise in the continua is between 150 and 250 .

Stellar parameters such as $T_{\mathrm{eff}}, \log g,[\mathrm{M} / \mathrm{H}]$, and $v \sin i$ have been determined by a comparison of the observed spectra with synthetic ones, where we used the spectral range 4740 to $5800 \AA$, which is almost free of telluric contributions. The synthetic spectra have been computed with the SynthV programme (Tsymbal 1996) based on atmosphere models computed with LLmodels (Shulyak et al. 2004). Scaled solar abundances have been used for different values of $[\mathrm{M} / \mathrm{H}]$. A detailed description of the applied method can be found in Lehmann et al. (2011). The resulting values of $T_{\text {eff }}, \log g$ and $v \sin i$ are presented in Table 2, under label " $g$ ". Errors are determined from $\chi^{2}$ statistics and represent a 1- $\sigma$ confidence level. Detailed analysis results, including also values of $[\mathrm{M} / \mathrm{H}]$ and microturbulent velocity, will be published in a dedicated paper (Tkachenko et al., in prep.).

\section{Characterization of the sample}

Figure 2 shows the distribution of the 750 sample stars in $T_{\text {eff }}$ (top left), $\log g$ (top right), Kepler magnitude Kp (bottom left), and total length of the Kepler light curve $\Delta T$, expressed in $\mathrm{d}$ (bottom right). For the analysis we used $T_{\text {eff }}$ and $\log g$ values given in boldface in Table 2. Note that seven stars in our sample are hotter than $T_{\text {eff }}=9000 \mathrm{~K}$, and fall off the diagram.

The following typical global parameters have been observed for $\delta$ Sct and $\gamma$ Dor stars (e.g. Rodríguez \& Breger 2001; Handler $\&$ Shobbrook 2002): $\log g=3.2-4.3$ and $T_{\text {eff }}=6300-8600 \mathrm{~K}$ for $\delta$ Sct stars, and $\log g=3.9-4.3$ and $T_{\text {eff }}=6900-7500 \mathrm{~K}$ for $\gamma$ Dor stars. While $\gamma$ Dor stars are generally MS stars, several more evolved $\delta$ Sct stars have been observed.

The distributions in Fig. 2 show that about $70 \%$ of the total sample does indeed have $T_{\text {eff }}$ values between $6300 \mathrm{~K}$ and $8600 \mathrm{~K}$. However, a significant number (about 20\%) are cooler stars. The $\log g$ values of our sample are concentrated on 3.5-4.5, which represents about $76 \%$ of the total sample.

The sample consists of stars with magnitudes in the range $6<\mathrm{Kp}<15 \mathrm{mag}$. The majority (about 55\%) is located in the interval $\mathrm{Kp}=[10,12]$ mag. Given that stars with magnitudes fainter than $V=9$ are difficult to monitor spectroscopically from 

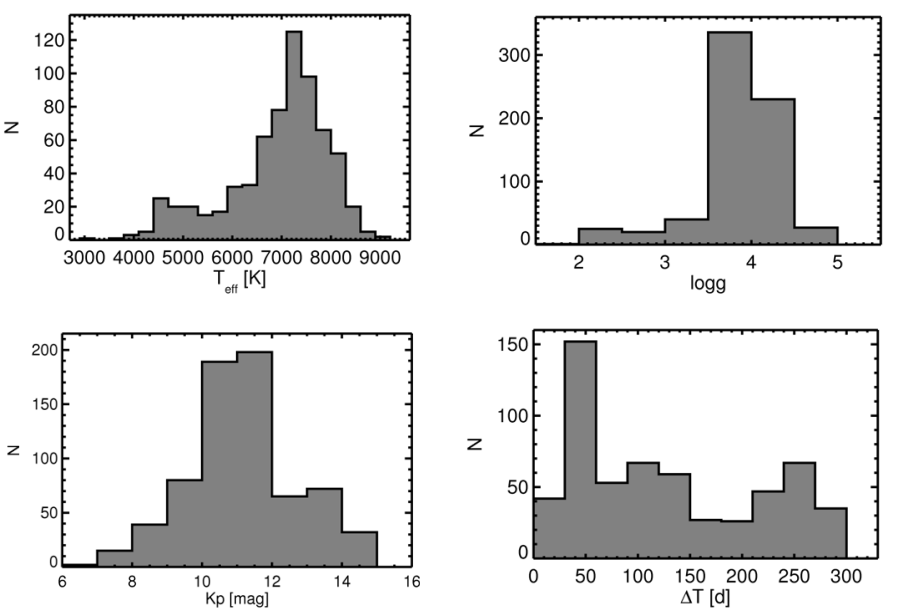

Fig. 2. Distribution in $T_{\text {eff }}$ (top left), $\log g$ (top right), Kepler magnitude Kp (bottom left), and total time span $\Delta T$ of the Kepler light curves, expressed in d (bottom right) of the 750 sample stars. The number of stars belonging to each bin $(N)$ is indicated on the $Y$-axis. We used the adopted values of $T_{\text {eff }}$ and $\log g$, as given in boldface in Table 2 .

the ground with 2 m-class telescopes, the fact that about $92 \%$ of the stars are fainter than $V=9$ has implications for the feasibility of possible spectroscopic ground-based follow-up observations (see Uytterhoeven et al. 2010a,b).

Finally, the total length of the Kepler dataset (not taking into account possible gaps of several tens of days) is spread between 9.5 and 322 d. For a considerable fraction (19\%) of the sample only Q0 and Q1 data are available, with a total length of $44 \mathrm{~d}$, implying a frequency resolution slightly worse than $0.02 \mathrm{~d}^{-1}$. On the other hand, 351 stars, or $47 \%$ of the total sample, have a time span of more than $200 \mathrm{~d}$ (resulting in a frequency resolution better than $0.005 \mathrm{~d}^{-1}$ ). Of these 351 stars, $46 \%$ have a maximum time gap in the light curve of less than $10 \mathrm{~d}$, and $23 \%$ have a gap of over $200 \mathrm{~d}$ and up to $325 \mathrm{~d}$.

In the following sections we will describe the variability analysis results of all 750 stars. At this stage we did not exclude any of the stars from the sample on grounds of non-compatibility of physical parameters with the current expectations for A- and F-type pulsators, to present a homogenous analysis and to investigate if Kepler confirms the current understanding of $\delta$ Sct and $\gamma$ Dor stars.

\section{Frequency analysis}

\subsection{Treatment of the Kepler light curves}

In this paper we used the "non-corrected" light curves available to KASC for asteroseismic investigations through the KASOC database. A description of the Kepler data reduction pipeline is given by Jenkins et al. (2010a,b). However, these raw time series suffer from some instrumental perturbations that need to be corrected for, e.g. perturbations caused by the heating and cooling down of the Kepler CCDs, variations caused by changes in the aperture size of the source mask, etc. Some of the effects are well known, and the corresponding non-stellar frequencies are tabulated by the Kepler team (e.g. frequencies near 32, 400, 430 , and $690 \mathrm{~d}^{-1}$ ). Other perturbations are not documented, and are harder to evaluate and correct for.

We subjected the light curves of all sample stars to an automated procedure that involves fitting a cubic spline to the time series, and correcting the residuals for discontinuities and outliers. To investigate if and to what extent artificial periodicities at the same timescale as the expected pulsations in $\gamma$ Dor and $\delta$ Sct stars are introduced by the correction, we also corrected a subsample of stars by a different procedure that takes three types of effects into account, namely outliers, jumps, and drifts (see García et al. 2011). Both correction methods gave the same frequency analysis results within the accuracy of the dataset.

Next, the Kepler flux $\left(F_{\mathrm{Kp}}(t)\right)$ was converted to parts-permillion $(\mathrm{ppm})\left(F_{\mathrm{ppm}}(t)\right)$, using the following formula:

$F_{\mathrm{ppm}}(t)=10^{6} \times\left(\frac{F_{\mathrm{Kp}}(t)}{\mathrm{f}(\mathrm{t})}-1\right)$,

with $f(t)$ a polynomial fit to the light curve. A test on the effect of the use of different polynomial orders (2 to 10) on the detected frequencies in the time series showed that, in general, a third or fourth order polynomial fits the overall curvature better than a linear fit. The choice of the polynomial did not change periodicities with frequencies higher than $0.2 \mathrm{~d}^{-1}$. The obtained error for frequencies between 0.01 and $0.2 \mathrm{~d}^{-1}$ was of the order of $1 / \Delta T \mathrm{~d}^{-1}$, with $\Delta T$ the total time span of the light curve expressed in $\mathrm{d}$.

\subsection{Frequency analysis}

The Kepler time series of the 750 sample stars were analysed in a homogenous way, using a programme based on the LombScargle analysis method (Scargle 1982). Frequencies were extracted in an iterative way until the Scargle false alarm probability (fap; Scargle 1982), a measure for the significance of a peak with respect to the underlying noise level, reached 0.001 . In view of the almost uninterrupted and equidistant sampling of the Kepler data, this estimate of the fap is a fast and reliable approximation of the true fap, because the number of independent frequencies can be estimated precisely (see also the discussion in Sect. 4 of Balona et al. 2011b). Frequencies were calculated with an oversampling factor of 10 . Time series consisting of only LC data were not searched for periods shorter than $1 \mathrm{~h}$, because the corresponding Nyquist frequency is $24 \mathrm{~d}^{-1}$. For SC data, with a time sampling of about $1 \mathrm{~min}$, frequencies up to $720 \mathrm{~d}^{-1}$ could be detected.

As a comparison, subsamples of the stars were analysed using different analysis methods, such as SigSpec (Reegen 2007, 2011), Period04 (Lenz \& Breger 2005), the generalized Lomb-Scargle periodogram (Zechmeister \& Kürster 2009), and the non-interactive code, freqfind (Leroy \& Gutiérrez-Soto, in prep.). The latter code is based on the non-uniform fast Fourier transform by Keiner et al. (2009), and significantly decreases the computation time for unevenly spaced data. The results obtained with the different methods were consistent.

\section{Classification}

\section{1. $\delta$ Sct, $\gamma$ Dor, and hybrid stars}

We performed a careful inspection (one-by-one, and by eye) of the 750 light curves, the extracted frequency spectra, and list of detected frequencies, and tried to identify candidate $\delta$ Sct, $\gamma$ Dor, and hybrid stars. We used a conservative approach and omitted frequencies with amplitudes lower than 20 ppm for the classification. We also filtered out obvious combination frequencies and harmonics ${ }^{2}$ in an automatic way, and only considered apparent independent frequencies for the analysis. We suspect that the

2 As obvious combination frequencies and harmonics we considered $n f_{i}$ or $k f_{i} \pm l f_{j}$, with $f_{i}$ and $f_{j}$ different frequencies, $n \in[2,3,4,5]$, and $k, l \in[1,2,3,4,5]$. 
variable signal of a few stars is contaminated by the light variations of a brighter neighbouring star on the CCD. We flagged all stars with a high contamination factor $(>0.15)$, as given by the KIC. If the light curves of the neighbouring stars on the CCD were available through $\mathrm{KASOC}^{3}$, we carefully checked the light curves of these stars with their neighbours. Stars that show an obvious contamination effect were omitted from classification. We used information on $T_{\text {eff }}$ (Table 2) to distinguish between $\delta$ Sct and $\gamma$ Dor stars versus $\beta$ Cep and SPB stars. To be conservative, low frequencies $\left(<0.5 \mathrm{~d}^{-1}\right)$ (see, for instance, the frequency spectra in Fig. 4) are currently not taken into account in the analysis, because in this frequency range real stellar frequencies are contaminated with frequencies resulting from instrumental effects (see Sect. 5.1), and the separation of the different origins requires a dedicated study, which is beyond the scope of this paper.

We encountered a variety of light curve behaviour. Based on a small number of stars and using only the first quarter of Kepler data, Grigahcène et al. (2010) already proposed a subdivision of the A-F type pulsators into pure $\delta$ Sct stars, pure $\gamma$ Dor stars, $\delta$ Sct $/ \gamma$ Dor hybrids and $\gamma$ Dor $/ \delta$ Sct hybrids, using the fact that frequencies are only detected in the $\delta$ Sct (i.e. $>5 \mathrm{~d}^{-1}$, or $>58 \mu \mathrm{Hz}$ ) or $\gamma$ Dor (i.e. $<5 \mathrm{~d}^{-1}$ or $<58 \mu \mathrm{Hz}$ ) domain, or in both domains with dominant frequencies in either the $\delta$ Sct star or $\gamma$ Dor star region, respectively. Among the 750 sample stars we see different manifestations of hybrid variability. There are stars that show frequencies with amplitudes of similar height in both regimes, and stars with dominant frequencies in the $\gamma$ Dor $(\delta \mathrm{Sct})$ domain and low amplitude frequencies in the $\delta$ Sct ( $\gamma$ Dor) domain. The light curves show diversity as well. Balona et al. (2011d) already commented on the different shapes of light curves of pure $\gamma$ Dor stars.

In this work, we focus on stars that show at least three independent frequencies. We classified the stars in three groups: $\delta$ Sct stars, $\gamma$ Dor stars, and hybrid stars. Because the underlying physics that causes the different types of hybrid behaviour is currently not clear, all types of hybridity (both $\delta$ Sct $/ \gamma$ Dor hybrids and $\gamma$ Dor $\delta$ Sct hybrids) are included in the group of hybrids. A star was classified as a hybrid star only if it satisfied all of the following criteria:

- frequencies are detected in the $\delta$ Sct (i.e. $>5 \mathrm{~d}^{-1}$ or $>58 \mu \mathrm{Hz}$ ) and $\gamma$ Dor domain (i.e. $<5 \mathrm{~d}^{-1}$ or $<58 \mu \mathrm{Hz}$ );

- the amplitudes in the two domains are either comparable, or the amplitudes do not differ more than a factor of 5-7 (case-to-case judgement);

- at least two independent frequencies are detected in both regimes with amplitudes higher than $100 \mathrm{ppm}$.

By using these criteria, we should reduce the number of false positive detections. In particular, we tried to avoid a hybrid star classification of "pure" $\delta$ Sct stars that show a prominent longterm variability signal caused by rotation. We also tried to take care of more evolved $\delta$ Sct stars that are expected to pulsate with frequencies lower than $5 \mathrm{~d}^{-1}$. Stars that exhibited only or mainly frequencies in the $\delta$ Sct domain (i.e. $>5 \mathrm{~d}^{-1}$ ) and did not satisfy all of the above given criteria were assigned to the pure $\delta$ Sct group. Likewise, the group of pure $\gamma$ Dor stars consists of stars that do not comply with the hybrid star criteria, and that

\footnotetext{
${ }^{3}$ Unfortunately, only 40 stars of the sample could be checked in this way. We saw a clear contamination for the stars KIC 4048488 and KIC 4048494, KIC 5724810 and KIC 5724811, and KIC 3457431 and KIC 3457434. Less clear contamination is seen for KIC 4937255 and KIC 4937257, and KIC 10035772 and KIC 10035775, which are stars that show no obvious periodic variable signals.
}

have only or mainly frequencies lower than $5 \mathrm{~d}^{-1}$. However, the classification of pure $\gamma$ Dor stars is not as straightforward, because several other physical processes and phenomena can give rise to variability on similar timescales, such as binarity and rotational modulation caused by migrating star spots. We tried our best to select only $\gamma$ Dor stars, but are aware that nonetheless, and most likely, our selection is contaminated with a few non-bona fide $\gamma$ Dor stars. For stars that were observed in nonconsecutive Kepler quarters, we tried to beware of frequencies introduced by the spectral window. For instance, frequently a peak near $48 \mathrm{~d}^{-1}(555 \mu \mathrm{Hz})$ is detected (e.g. KIC 2166218 and KIC 7798339), which for a $\gamma$ Dor pulsator can result in an incorrect classification as hybrid star.

In Figs. 3-5 a portion of the light curve with a time span of $2 \mathrm{~d}$ ( $\delta$ Sct stars) or $5 \mathrm{~d}$ ( $\gamma$ Dor and hybrid stars) and a schematic overview of the detected independent frequencies (i.e. combination frequencies are filtered out in an automated way, see above) are given for a few representative stars of each group. The amplitudes and Kepler flux are expressed in ppm, and the frequencies are given in both $\mathrm{d}^{-1}$ (bottom $X$-axis) and $\mu \mathrm{Hz}$ (top $X$-axis). The dotted grey line in the amplitude spectra separates the $\delta$ Sct and $\gamma$ Dor regime. The dates are in the Heliocentric Julian Date (HJD) format $\mathrm{HJD}_{0}=2454$ 950.0. The figures illustrate the variety of pulsational behaviour within the groups. The $\delta$ Sct stars (Fig. 3) display an impressive variety of amplitude heights. The variability of the stars in panels (d) and (e), KIC 9845907 and KIC 9306095, respectively, is dominated by one high-amplitude frequency. Several lower amplitude variations are also present. The chance of confusing a high amplitude $\delta$ Sct star (HADS) and binarity is high for KIC 9306095. The stars in panels (a)-(c) show multiperiodic variations with frequency amplitudes of similar size. The rotational frequency near $1.2 \mathrm{~d}^{-1}$ and its first harmonic of the star KIC 10717871 (panel c) could be mistaken for $\gamma$ Dor-like frequencies. Because there are no other longer-term periodicities, there is no evidence for the possible hybrid status of this star.

The light curves of $\gamma$ Dor stars (Fig. 4) vary from obvious beat patterns to less recognizable variable signals. Balona et al. (2011d) already pointed out that there are symmetric (e.g. panel d) and asymmetric (e.g. panel e) light curves among the stars that show obvious beating, and that most likely in these cases the pulsation frequencies are comparable to the rotation frequency. Balona et al. (2011d) also suggested that the more irregular light curves likely stem from slowly rotating stars.

Examples of hybrid stars are given in Fig. 5. The grey dotted line in the right panels guide the eye to separate the $\delta$ Sct and $\gamma$ Dor regimes. The stars KIC 3119604 and KIC 2853280 (panels a and b, respectively) are clearly dominated by $\delta$ Sct frequencies, while the $\gamma$ Dor frequencies have lower amplitudes. The star KIC 9664869 (panel c) is an example of a star that exhibits frequencies with amplitudes of comparable height in the two regimes. The highest peak in the $\gamma$ Dor region is most likely related to the stellar rotation period, however, because several harmonics are also observed. The bottom two panels are examples of hybrid stars dominated by $\gamma$ Dor periodicities.

Table 3, available in the on-line version of the paper, presents an overview of the stars assigned to the three groups. For each star (KIC ID) we provide the classification (Class), the total number of independent frequencies $(N)$ detected above the significance level (fap $=0.001$ ) and with amplitudes higher than $20 \mathrm{ppm}$, and the number of independent frequencies detected in the $\gamma$ Dor and $\delta$ Sct regime $\left(N_{\gamma \text { Dor }}\right.$ and $N_{\delta \text { Sct }}$, respectively). The next column gives as a reference the total number 

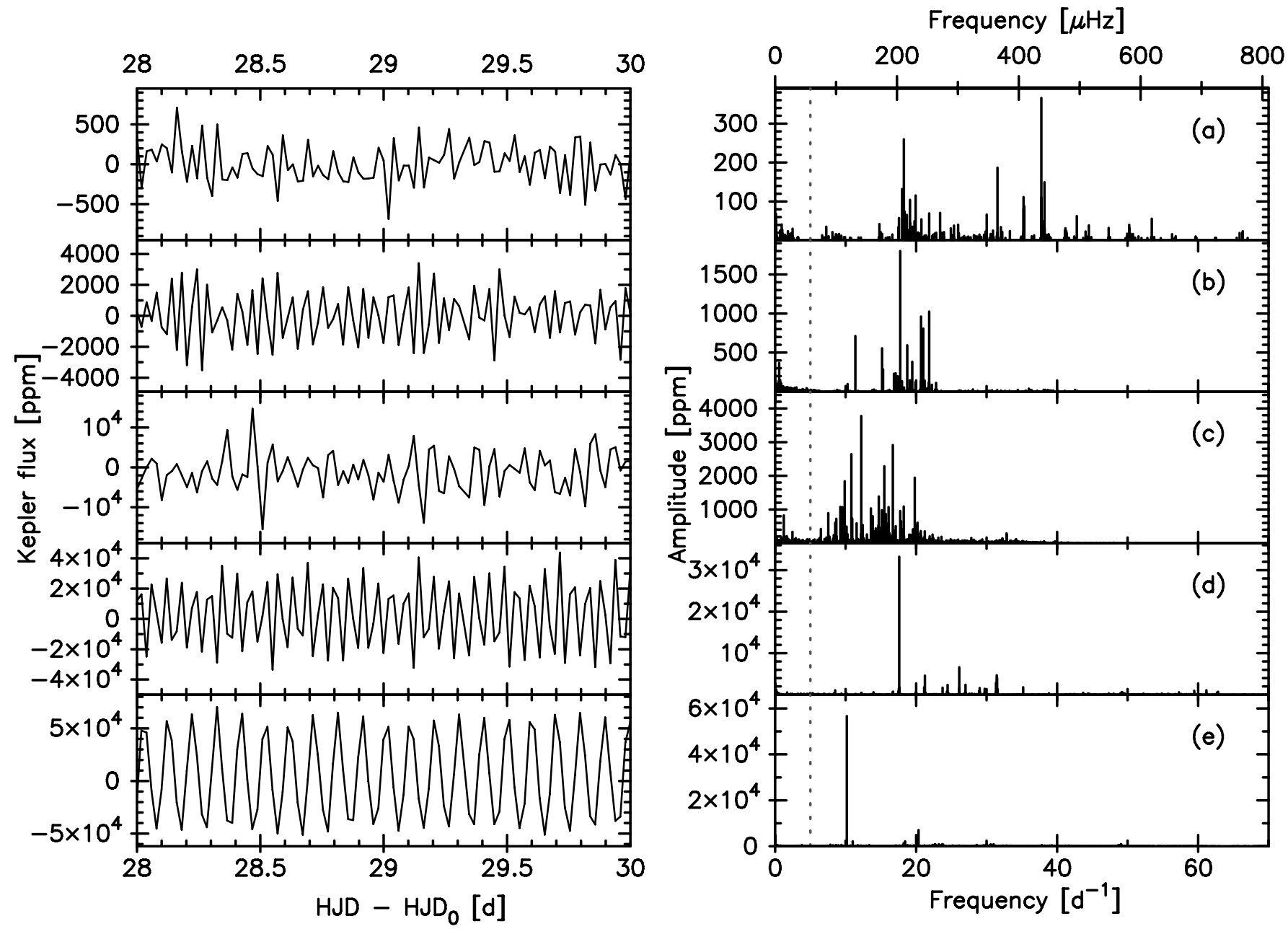

Fig. 3. Light curve and frequency spectrum of five stars assigned to the $\delta$ Sct group, illustrating the variety of pulsational behaviour within the group. The left panel shows a portion of the Kepler light curves. The Kepler flux is expressed in ppm, HJD is given in d with respect to HJD $\mathrm{D}_{0}=$ 2454 950.0. The right panel gives a schematic representation of the detected independent frequencies, expressed in $\mathrm{d}^{-1}$ (bottom $X$-axis) or $\mu \mathrm{Hz}$ (top $X$-axis). Amplitudes are given in ppm. The dotted grey line separates the $\delta$ Sct and $\gamma$ Dor regime. Note the different $Y$-axis scales for each star. a) KIC 8415752; b) KIC 8103917; c) KIC10717871; d) KIC 9845907; e) KIC 9306095.

of frequencies detected above the significance level, including combination frequencies and harmonics $\left(N_{\text {total }}\right)$. The next four columns denote the frequency range of peaks in the $\gamma$ Dor and $\delta$ Sct regimes ((Freq Range) ${ }_{\gamma \text { Dor }}$ and (Freq Range) $)_{\delta \text { Sct }}$, expressed in $\mathrm{d}^{-1}$ ), the highest amplitude (Amplitude ${ }_{\text {high }}$, expressed in ppm) and associated frequency $\left(\right.$ Freq $_{\text {high }}$, in $\mathrm{d}^{-1}$ ). In the last column a flag $(\bullet)$ indicates if the risk on light contamination with a neighbouring star on the CCD is high (contamination factor $>0.15$ ). A typical error on the frequency associated with the highest amplitude is $0.0001 \mathrm{~d}^{-1}$. The error on the amplitude ranges from a few ppm up to about $30 \mathrm{ppm}$. We note that for stars identified as $\gamma$ Dor or $\delta$ Sct stars we report on frequencies up to $6 \mathrm{~d}^{-1}$ or from $4 \mathrm{~d}^{-1}$, respectively, to account for, for instance, the frequency spectrum of more evolved stars.

We note that for several stars classified as $\gamma$ Dor star only LC data are available. This may create a selection effect, because short-term $\delta$ Sct periods are more difficult to detect in the short timestring of LC data owing to sampling restrictions. Also, as mentioned above, even though we carefully checked the stars one by one, we expect to have a few false positive detections of hybrid and $\gamma$ Dor stars because the typical $\gamma$ Dor frequencies can be easily confused with variations of the order of a day caused by rotation or binarity. A more careful analysis and interpretation of the full frequency spectrum of all individual stars of the sample will clarify this matter, but this is beyond the scope of this paper.

We compared our classification with the automated supervised classification results presented by Debosscher et al. (2011). Because these authors studied public Kepler Q1 data, only 479 objects of our sample appear in their catalogue. We point out that the classifier by Debosscher et al. (2009) only takes three independent frequencies with the highest amplitudes into account. Hence, the recognition and classification of hybrid behaviour is currently not implemented. Moreover, because the classifier does not take external information into account that can distinguish between B-type stars and A-F type stars (e.g. colour information, spectral classification based on spectra), there is often a confusion between $\delta$ Sct and $\beta$ Cep stars, and between $\gamma$ Dor and SPB stars. In general, there is good agreement (>87\%, classified in terms of $\delta$ Sct or $\beta$ Cep stars) with the classification by Debosscher et al. (2011) for stars that we classified as $\delta$ Sct stars. The $\gamma$ Dor stars, as we classified them, are in general less easily recognized by the automated classifier. Often they appear as "miscellaneous" in their list. This is not surprising, because 

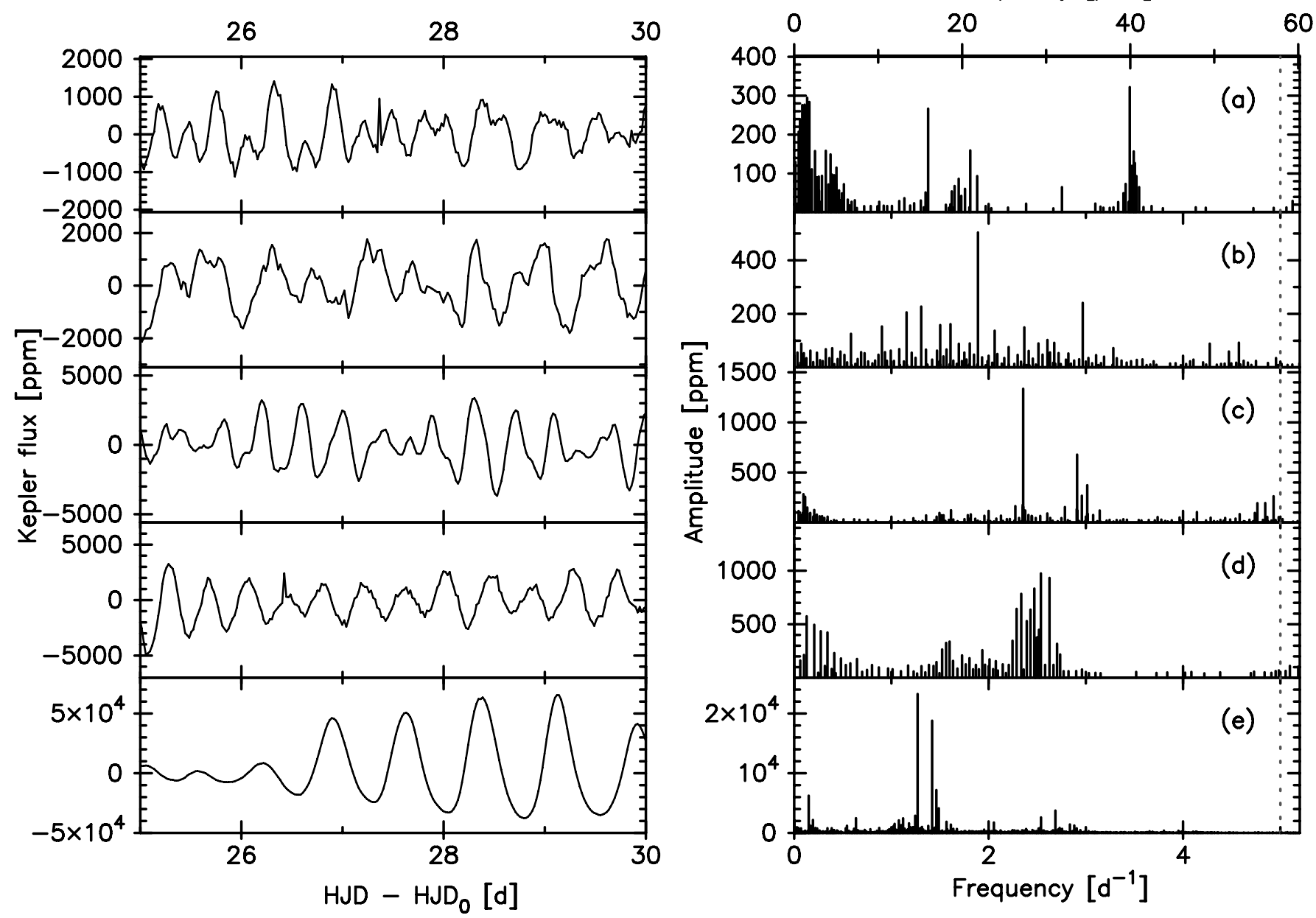

Fig. 4. Similar figure as Fig. 3, but for five candidate $\gamma$ Dor stars. Note the different $X$-axis scale with respect to Fig. 3. a) KIC 1432149; b) KIC 5180796; c) KIC 7106648; d) KIC 8330056; e) KIC 7304385.

so far only a few high-quality light curves of well-recognized $\gamma$ Dor stars were available that could be used as a template to feed the classifier. Stars that we identified as hybrid stars appear in the catalogue by Debosscher et al. (2011) as "miscellaneous" or as $\delta$ Sct, $\gamma$ Dor, $\beta$ Cep, or SPB stars. The work presented in this paper will provide valuable feedback and information to refine the automated supervised classification procedure developed by Debosscher et al. (2009).

\subsection{Other classes}

About $63 \%$ of our sample is recognized as $\delta$ Sct, $\gamma$ Dor, or hybrid star. Table 4 , in the on-line version of the paper, gives an overview of the "classification" of the remaining $37 \%$ of the stars. For each star (KIC ID) the associated classification (Class) and a flag (Flag) indicating a high risk on light contamination by a neighbouring star ( $\bullet$ if there is a contamination factor $>0.15$ ), are given. Table 4 includes stars that show no clear periodic variability on timescales typical for $\delta$ Sct and $\gamma$ Dor pulsators (“...", or "solar-like"), stars that exhibit stellar activity and show a rotationally modulated signal ("rotation/activity"), binaries ("binary" or eclipsing binary "EB"), B-type stars ("Bstar"), candidate red giant stars ("red giant"), Cepheids ("Cepheid"), and stars whose light is contaminated by another star ("contaminated"). Although the observed ranges in $T_{\text {eff }}$ and $\log g$ include typical values for RR Lyr stars (see Fig. 2), we did not find any in our sample, but there are $\sim 40$ such stars observed by Kepler, which are studied separately (Kolenberg et al. 2010; Benkó et al. 2010). Unclear cases mostly show a behaviour that might be related to rotation and are hence also labelled "rotation/activity". We also assigned the candidate $\gamma$ Dor stars for which less than three significant peaks were detected to this category. The light curve and frequency spectra of a few examples of these other classifications are given in Figs. 6 and 7.

One hundred and twenty-one stars do not show an obvious periodicity in the expected range for $\gamma$ Dor and $\delta$ Sct stars, or have an unresolved frequency spectrum within the available dataset. The star KIC 9386259 (Fig. 6, panel a) is an example of a star showing no clear periodicity. Furthermore, we used the label "..." for some stars for which less than three significant frequencies were detected (e.g. KIC 11509728 and KIC 11910256). We investigated the stars for signatures of solar-like oscillations and identified 75 candidate solar-like oscillators ("solar-like", see Table 4).

We identified seven B-type stars and 44 red giant stars in the sample. The giant stars show an envelope of frequencies with amplitudes up to $100-200 \mathrm{ppm}$ in the region $0.5-5 \mathrm{~d}^{-1}$, as illustrated by KIC 2584202 (Fig. 6, panel b). Among the B-type stars, we recognized five SPB stars and one candidate $\beta$ Cep star.

Within the sample we identified at least 39 binaries, including 28 EBs. In Table 4 the binary stars are labelled "binary", or "EB" for an EB. If the variability of one of the components 

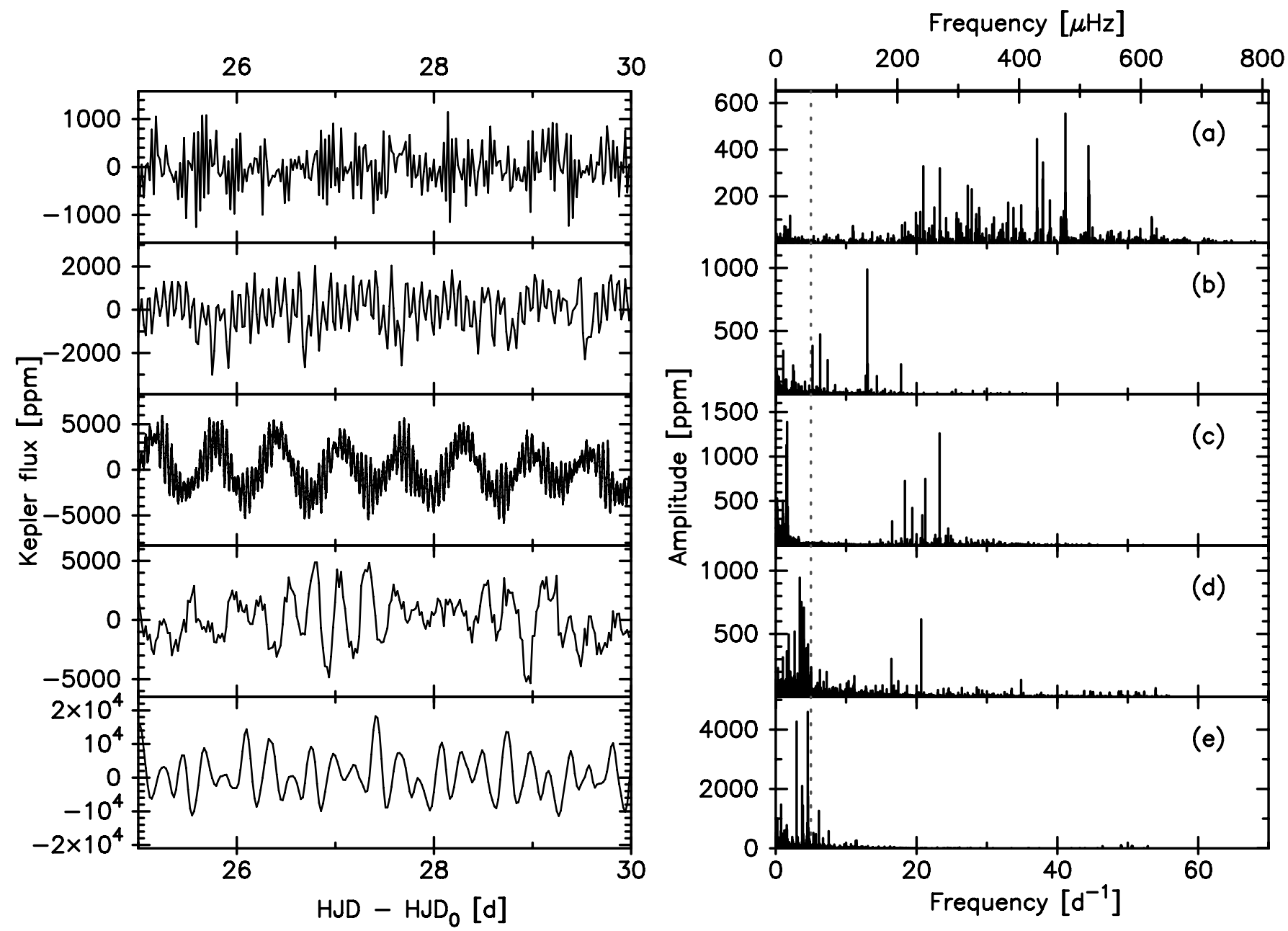

Fig. 5. Similar figure as Fig. 3, but for five candidate hybrid stars. a) KIC 3119604; b) KIC 2853280; c) KIC 9664869; d) KIC 9970568; e) KIC 3337002. The two top panel $\mathbf{a})-\mathbf{b})$ are $\delta$ Sct frequency-dominated stars, and the two bottom panels d)-e) are $\gamma$ Dor frequency-dominated stars.

is identified as typical for one of the three groups outlined in Sect. 6.1, we also indicated this in Table 4. Panels (c)-(e) of Fig. 6 show examples of EBs. An interesting target is KIC 11973705 , because it most likely is a binary with a $\delta$ Sct and SPB component (see also Balona et al. 2011b). For three stars reported in the literature as EBs (Prša et al. 2011; Slawson et al. 2011; Hartman et al. 2004), KIC 2557115, KIC 5810113, and KIC 1432149, we find no clear evidence of their eclipsing nature in the Kepler lightcurves. In case of KIC 1432149, presented by Hartman et al. (2004) as an EB with period $9.3562 \mathrm{~d}$, we cannot confirm its eclipsing nature or its orbital period, and we suspect that this target has been misidentified as an EB.

Several stars show an irregular light curve typical of stellar activity, or a clearly rotationally modified signal (panels (a)-(c) of Fig. 7). It is also not impossible that low-amplitude pulsating $\gamma$ Dor star candidates are hidden among the stars labelled as "rotation/activity" in Table 4. Namely, when only one or two of their pulsation frequencies reach the current detection threshold, they are not yet assigned to a pulsation group. A possible $\gamma$ Dor candidate is given in panel (d) of Fig. 7 .

In some cases the light curves look very peculiar, and the origin of the variability is not clear. This is the case for KIC 3348390 (panel (e) of Fig. 7) and KIC 4857678, for instance.
We discovered several interesting targets among the 750 stars of the sample. Dedicated studies of groups of individual stars will be presented in forthcoming papers. Below, we will sort the stars into different classes.

\section{Characterization of the different classes}

The classification described in the previous section results in the following distribution. A total of $63 \%$ of the sample can be identified as $\gamma$ Dor, $\delta$ Sct or hybrid stars: $27 \%$ are classified as $\delta$ Sct stars (206 stars), 23\% as hybrid stars (171 stars; of which 115 stars are $\delta$ Sct-dominated and 56 stars are $\gamma$ Dor-dominated), and $13 \%$ as $\gamma$ Dor stars (100 stars). A striking result is that almost a quarter of the sample, i.e. 171 stars, shows hybrid behaviour. This is in sharp contrast with the results obtained from ground-based observations, where so far only three candidate $\gamma$ Dor- $\delta$ Sct hybrid stars have been discovered. The far superior precision of the space data opens a new window in detecting low amplitude variations. This result was already hinted at by Grigahcène et al. (2010) and Hareter et al. (2010), but the quantification by means of this sample is remarkable.

Of the remaining $37 \%$ of the sample, a considerable number (121 stars, 16\%) do not show clear variability with periods in the expected range for $\gamma$ Dor and $\delta$ Sct stars. Among this group are 

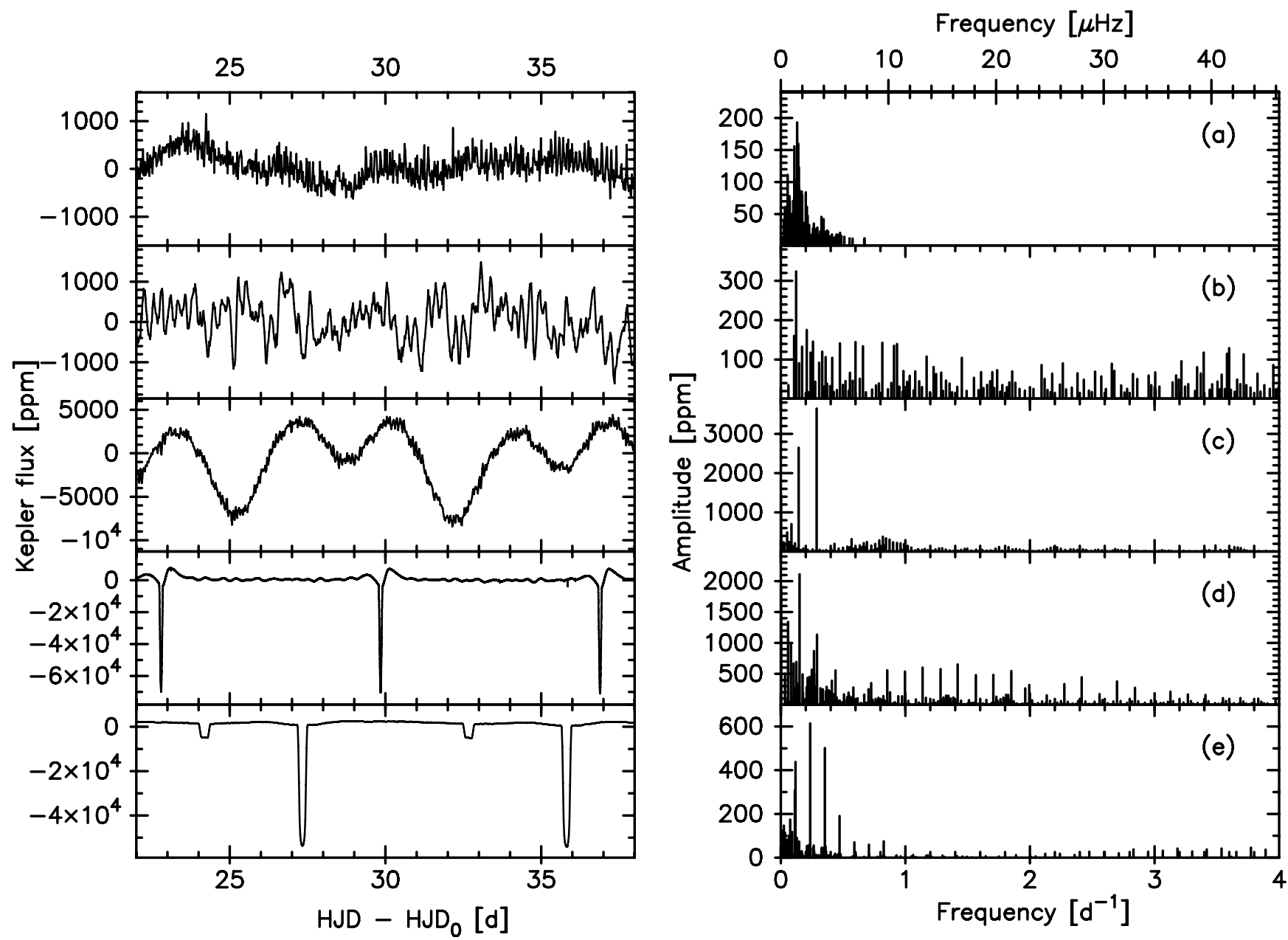

Fig. 6. Similar figure as Fig. 3, but for stars that were not assigned to the groups of $\delta$ Sct, $\gamma$ Dor or hybrid stars. a) KIC 9386259, no clear periodic signal detected; b) KIC 2584202, red giant star; c) KIC 5197256, EB or ellipsoidal variable with a $\delta$ Sct component; d) KIC 3230227, EB with a $\gamma$ Dor component; e) KIC 9851142, EB with most likely a $\gamma$ Dor component.

75 candidate solar-like oscillators. Our sample has seven B-type stars $(1 \%)$ and 44 stars $(6 \%)$ are identified as red giant stars. One Cepheid turned out to be among the sample. About $8 \%$ of the sample shows stellar activity, often manifesting itself by a rotationally modulated signal.

At least $5 \%$ of the sample stars are identified through the analysis of their light curve as binary or multiple systems, of which $3.5 \%$ show eclipses. When we also consider the known binaries from the literature (Table 1), we arrive at a binary rate of $12 \%$ within the sample. The number of binary detections is only a fraction of what is expected. The binary rate among A-F type stars in general and $\delta$ Sct stars in particular is estimated to be at least $30 \%$ (Breger \& Rodríguez 2000; Lampens \& Boffin 2000). Several additional stars are expected to be part of multiple systems with possibly much longer periods than the available Kepler time span. The percentage of EBs in our sample is high. Prša et al. (2011) reported a 1.2\% occurence rate of EBs among the Kepler targets.

Figure 8 shows the stars that are not assigned to one of the $\delta$ Sct, $\gamma$ Dor or hybrid star groups in a $\left(T_{\text {eff }}, \log g\right)$-diagram. The solid thick black and light grey lines mark the blue and red edge of the observed instability strip of $\delta$ Sct and $\gamma$ Dor stars, respectively (Rodríguez \& Breger 2001; Handler \& Shobbrook 2002). Owing to the possibly incorrect separation of the binary component's contribution, we considered the physical parameters of the binaries as insufficiently constrained and omitted them. The same holds for the B-type stars, which are much hotter than the $T_{\text {eff }}$ region shown here. The stars that show no clear periodic variability on timescales typical for $\delta$ Sct and $\gamma$ Dor pulsators (open triangles) and stars that exhibit stellar activity (bullets) are found along the MS and in more evolved stars. The location of the only Cepheid in our sample is marked by a cross. The candidate red giants (open squares) are all but one found in the expected region of the $\left(T_{\text {eff }}, \log g\right)$-diagram. This implies that the KIC photometry separates giant from MS stars well.

\subsection{Characterization of stars that show no clear periodic variability}

We now focus on the properties of the 121 stars that show no clear periodic variability in the $\gamma$ Dor and $\delta$ Sct range of frequencies to understand why no oscillations are detected. Figure 9 presents the distribution in $T_{\text {eff }}$ (top left), $\log g$ (top right), Kp (bottom left), and total time span $\Delta T$, expressed in $\mathrm{d}$, of the Kepler light curves (bottom right).

The cool boundary of the observational instability strip for $\gamma$ Dor stars is located around $T_{\text {eff }}=6900 \mathrm{~K}$. At least ${ }^{4} 78 \%$ of the 121 stars have cooler temperatures, and hence no A-F type variability is expected. About 75 stars are identified as candidate

${ }^{4}$ For $11 \%$ of the 121 stars we have no information on $T_{\text {eff }}$ or $\log g$. 

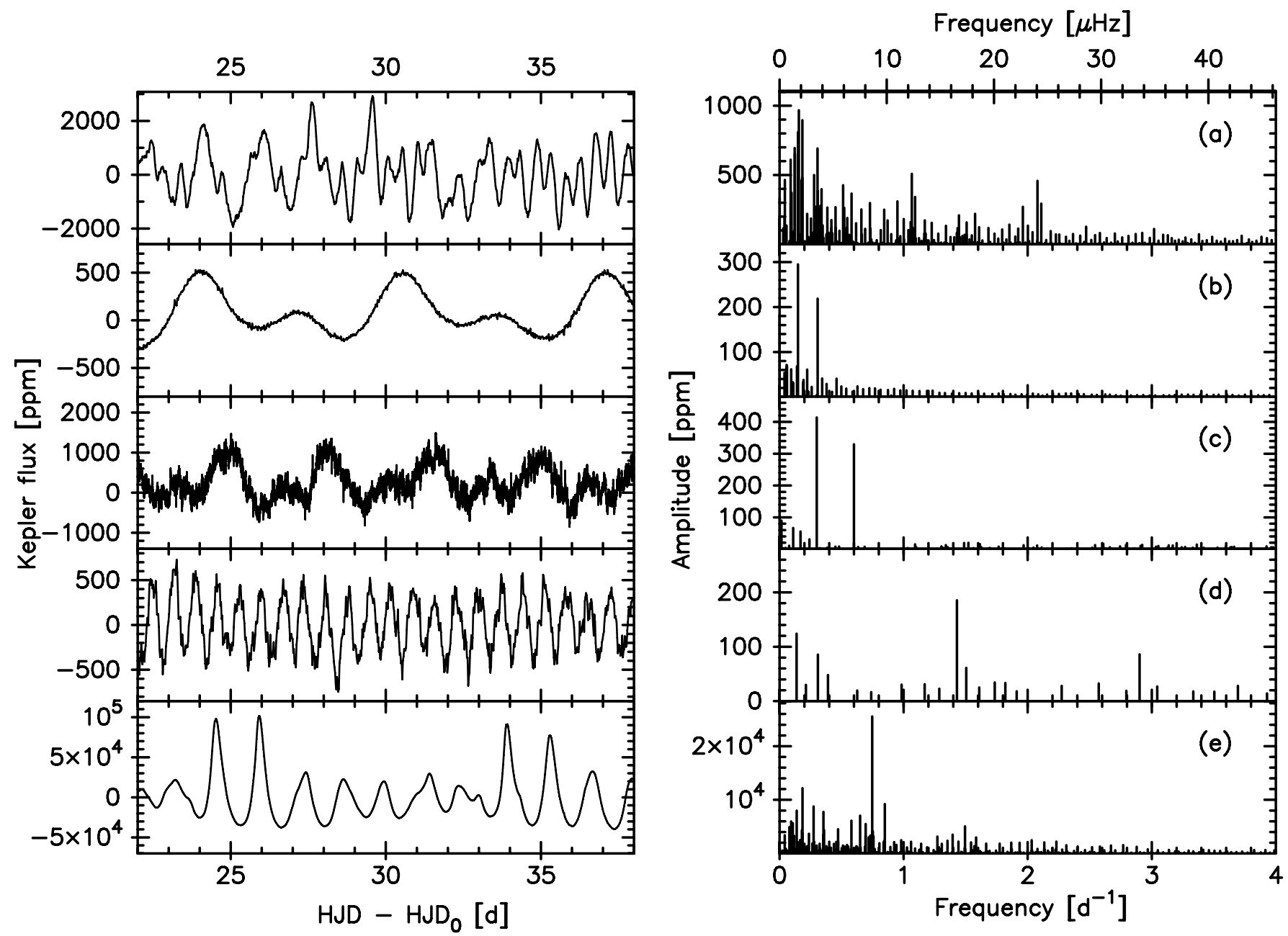

Fig. 7. Similar figure as Fig. 3, but for stars that were not assigned to the groups of $\delta$ Sct, $\gamma$ Dor or hybrid stars. Stellar activity/rotational modulation: a) KIC 8748251; b) KIC 8703413 and c) KIC 11498538; no clear classification: d) KIC 12062443; and e) KIC 3348390.

solar-like oscillators. However, $10 \%$ of the 121 stars that show no clear periodicity are located inside the instability strip of $\gamma$ Dor or $\delta$ Sct $\operatorname{stars}^{5}$ (see also Fig. 8). Additional investigation is needed to confirm that these stars do not show variability, which would imply that non-variable stars exist in the instability strip.

Sixty percent (71 stars) of our non-variable stars are fainter than $\mathrm{Kp}=12 \mathrm{mag}$, and $18 \%$ are fainter than $\mathrm{Kp}=14 \mathrm{mag}$. The faintness of the star most likely has an impact on the (non-)detection of periodicities. To quantify this, we counted the fraction of apparently non-periodic stars per magnitude bin for the full sample of 750 stars. The number of stars that show no clear peridodicity increases dramatically towards faint stars: the fraction is only $2 \%$ for magnitude $\mathrm{Kp}=9 \mathrm{mag}, 5 \%$ for $\mathrm{Kp}=10 \mathrm{mag}, 12 \%$ for $\mathrm{Kp}=11 \mathrm{mag}, 15 \%$ for $\mathrm{Kp}=12 \mathrm{mag}$, $41 \%$ for $\mathrm{Kp}=13 \mathrm{mag}$, and $68 \%$ for $\mathrm{Kp}>14 \mathrm{mag}$. The fainter the star, the more difficult it becomes to detect periodicities. Our analysis results, which were obtained by only considering amplitudes above $20 \mathrm{ppm}$, lead us to suspect that the Kepler detection limit of A-F type low-amplitude oscillations ( $\leq 20 \mathrm{ppm})$ lies around $\mathrm{Kp}=14$ mag (see also Sect. 7.2).

We find no evidence for a selection effect towards stars with a short time span in the available Kepler time series. The right panel of Fig. 9 shows that also several time series with long time

\footnotetext{
5 As demonstrated in Sect. 7.2, a revision of the current instability strip is required.
}

spans do not show clear variability. Also, the observing mode has no obvious influence on the (non-)detection of oscillations. Fifty-four percent of the 121 stars have only LC data, while $46 \%$ have only SC data.

To summarize, stars that show no clear periodic variations are generally the cooler and fainter stars of the sample. We do not find evidence for a bias towards the total time span of the available light curve or towards the observing mode (LC versus SC).

\subsection{Characterization of $\delta$ Sct, $\gamma$ Dor, and hybrid stars}

\subsubsection{The $\left(T_{\text {eff }}, \log g\right)$-diagram}

The current ground-based (GB) view on the positions of the $\delta$ Sct and $\gamma$ Dor classes in the $\left(T_{\text {eff }}, \log g\right)$-diagram (parameters are taken from the literature ${ }^{6}$ ) is presented in panel (a) of Fig. 10. A comparison of $\log g$ values derived from Geneva photometry and from other sources (photometry and spectroscopy)

${ }^{6}$ Rodríguez \& Breger (2001); Rodríguez et al. (2000); Henry \& Fekel (2005); Poretti et al. (1997); Breger et al. (1997); Zerbi et al. (1997, 1999); Aerts et al. (1998); Kaye et al. (1999b); Gray \& Kaye (1999); Eyer \& Aerts (2000); Guinan et al. (2001); Aerts (2001); Martín et al. (2003); Mathias et al. (2004); Rowe et al. (2006); Bruntt et al. (2008); Cuypers et al. (2009); Uytterhoeven et al. (2008); Catanzaro et al. $(2010,2011)$. 


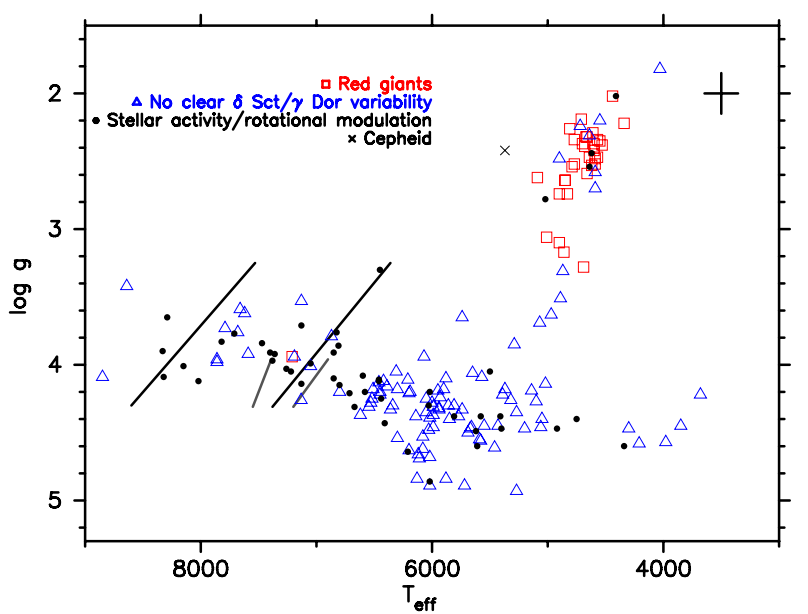

Fig. 8. $\left(T_{\text {eff }}, \log g\right)$-diagram with stars that show no clear periodic variability on timescales typical for $\delta$ Sct and $\gamma$ Dor pulsators (open triangles), stars identified as red giants (open squares), stars that exhibit stellar activity (bullet), and a Cepheid (cross). The cross at the right top corner represents the typical error bars on the values: $290 \mathrm{~K}$ for $T_{\text {eff }}$ and 0.3 dex for $\log g$. The solid thick black and light grey lines mark the blue and red edge of the observed instability strips of $\delta$ Sct and $\gamma$ Dor stars, as described by Rodríguez \& Breger (2001) and Handler \& Shobbrook (2002), respectively. In the on-line version of the paper the open squares, open triangles, bullets, and crosses, are red, blue, black, and black, respectively.
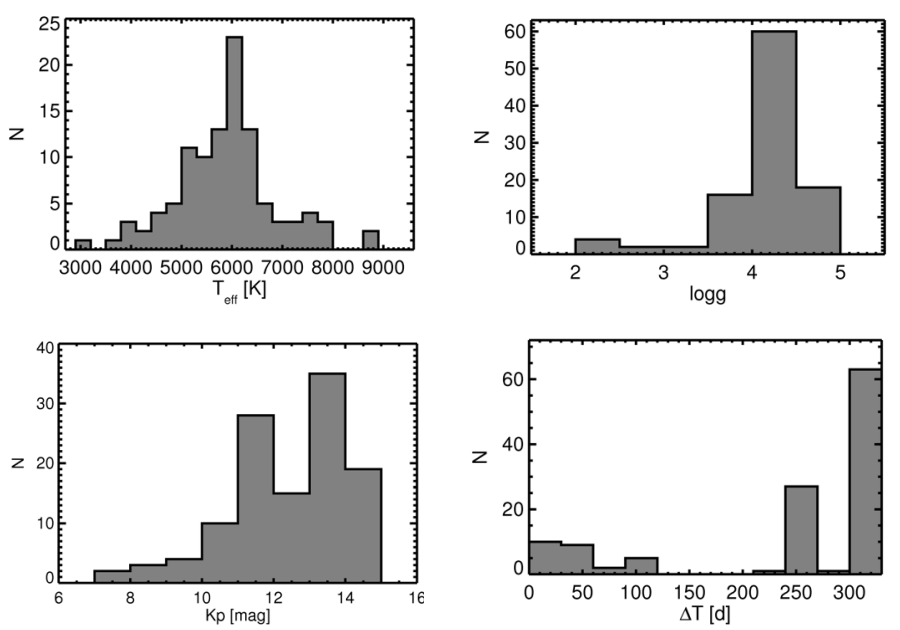

Fig. 9. Distribution in $T_{\text {eff }}$ (top left), $\log g$ (top right), Kepler magnitude Kp (bottom left), and total time span $\Delta T$ of the Kepler light curves (bottom right) of the 121 stars that show no clear periodic varibility. The number of stars belonging to each bin is given on the $Y$-axis.

indicates a systematic difference of about 0.4 dex for $\log g$ values above 4.35 dex as calculated from the Geneva photometry (Cuypers \& Hendrix, priv. comm.). Therefore we have corrected the values based on Geneva photometry. Evidently, the $\delta$ Sct and $\gamma$ Dor stars occupy distinct locations in the $\left(T_{\text {eff }}, \log g\right)$-diagram, with a small overlap region.

Panel (b) of Fig. 10 shows a different picture. Here the $\delta$ Sct, $\gamma$ Dor, and hybrid stars from the Kepler sample are plotted. We used the adopted values of $T_{\text {eff }}$ and $\log g$, as given in Table 2 . The cross in the top right corner of the figure shows typical errors on the values. The stars are scattered in the $\left(T_{\text {eff }}, \log g\right)$-diagram: the $\delta$ Sct and $\gamma$ Dor stars are not confined anymore to the two regions that were clearly seen for the ground-based stars. Even when considering the large error bars on the values, the scatter is present. Kepler $\delta$ Sct stars exist beyond the red edge of the instability strip, while Kepler $\gamma$ Dor pulsations appear in both hotter and cooler stars than previously observed from the ground. The Kepler hybrid stars occupy the entire region between the blue edge of the $\delta$ Sct instability strip and the red edge of the $\gamma$ Dor instability strip, and beyond. The position of the Kepler $\delta$ Sct and $\gamma$ Dor stars suggests that the edges of the so far accepted observational instability strips need to be revised. However, we need accurate values of $T_{\text {eff }}$ and $\log g$ for all stars to confirm this finding.

Because for most stars in our sample only KIC-based estimates of $T_{\text {eff }}$ and $\log g$ are available, we selected the stars that have reliable estimates of these parameters derived from groundbased spectra or multi-colour photometry (see Sect. 3). From this selection, 69 are classified as belonging to one of the three groups. The subsample of 69 stars is plotted in panel (c) of Fig. 10. The position of the stars in the $\left(T_{\text {eff }}, \log g\right)$-diagram confirms the general findings described for the full sample. However, the scatter across the diagram of $\gamma$ Dor stars is less present, but almost all $\gamma$ Dor candidates lie outside the observational instability strip for $\gamma$ Dor stars. Ground-based observations for the derivation of more precise values of $T_{\text {eff }}$ and $\log g$ are needed for all other stars to confirm the exact locations of the stars.

Panel (d) of Fig. 10 shows the Kepler stars assigned to the three groups that have amplitudes higher than $1000 \mathrm{ppm}$ (see Table 3), which approximately corresponds to amplitudes higher than $1 \mathrm{mmag}$ and hence might be observable from the ground. We notice that the Kepler stars with ground-based observable amplitudes also do not fit within the observational instability strips.

The left column of Fig. 11 presents an overview of the distribution in $T_{\text {eff }}$ for the three groups of A-F type stars. The histograms related to $\delta$ Sct, hybrid, and $\gamma$ Dor stars are coloured in dark grey, middle grey, and light grey respectively. The distribution in $T_{\text {eff }}$ peaks around $7400 \mathrm{~K}, 7200 \mathrm{~K}$, and $7000 \mathrm{~K}$ for $\delta$ Sct, hybrid, and $\gamma$ Dor pulsators, respectively. Comparing these values with the center of the observed instability strips by Rodríguez \& Breger (2001) and Handler \& Shobbrook (2002), we find that a large part of the Kepler stars are concentrated near the overlap of the two instability strips, and that many members of the three groups coincide in the same region in the $\left(T_{\text {eff }}, \log g\right)$-diagram. It will be interesting to investigate why stars with similar values of $T_{\text {eff }}$ and $\log g$ in some cases pulsate as a $\delta$ Sct star, and in others as a $\gamma$ Dor star, or as both. Another interesting and puzzling result is that $\gamma$ Dor and $\delta$ Sct pulsations seem to be excited in a far wider range of temperatures then previously expected.

The distribution in $\log g$ is similar for all classes. Most stars have $\log g$ values between $3.5 \mathrm{dex}$ and $4.3 \mathrm{dex}$, with a peak around $\log g=3.9$ dex. We point out that the $\log g$ values derived from the KIC for A-F type stars are known to have large uncertainties, and only few stars have measurements from other sources. Without more stars with accurate values derived from ground-based observations we cannot draw any conclusions.

The distribution in Kepler magnitude Kp (bottom left, Fig. 2) is representative for the distribution in Kp for $\gamma$ Dor and $\delta$ Sct stars. It illustrates that the cut-off magnitude for the detection of $\gamma$ Dor and $\delta$ Sct type of variations with Kepler lies around $\mathrm{Kp}=14$ mag. The majority of the sample stars have magnitudes in the range $\mathrm{Kp}=10-12 \mathrm{mag}$.

$v \sin i$ values are available for 41 stars of the subsample consisting of $\delta$ Sct, $\gamma$ Dor and hybrid stars (see Table 2). Of the five $\gamma$ Dor stars, four have $v \sin i$ values above $90 \mathrm{~km} \mathrm{~s}^{-1}$, and one has $v \sin i=15 \mathrm{~km} \mathrm{~s}^{-1}$. Of the sixteen $\delta$ Sct stars, 
K. Uytterhoeven et al.: The Kepler characterization of the variability among A- and F-type stars. I.
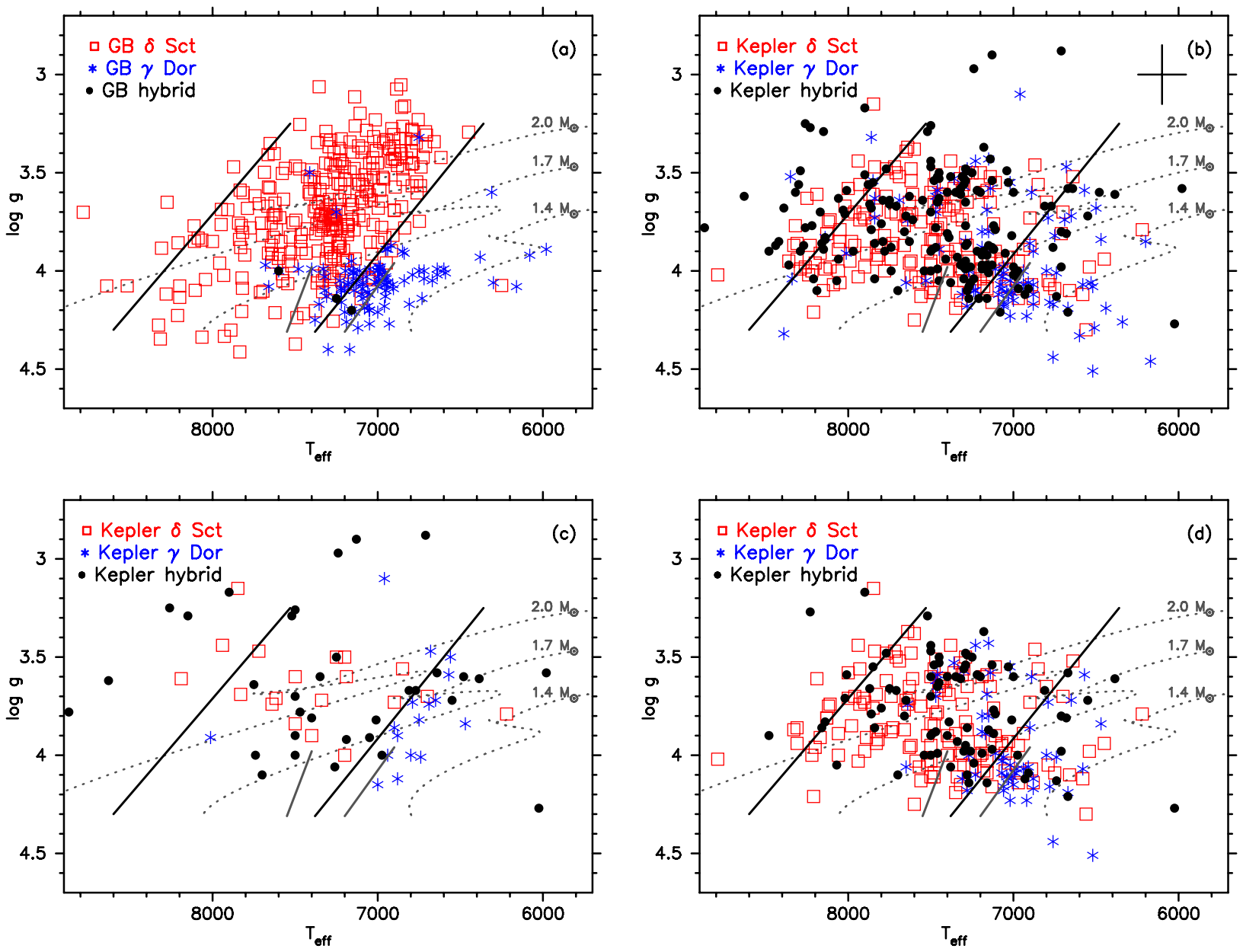

Fig. 10. a) $\left(T_{\text {eff }}, \log g\right.$ )-diagram of the $\delta \mathrm{Sct}, \gamma$ Dor, and hybrid stars detected from the ground (parameters taken from the literature). b) $\left(T_{\text {eff }}, \log g\right)$-diagram of the Kepler stars we classified as $\delta$ Sct, $\gamma$ Dor, and hybrid stars in this paper. Open squares represent $\delta$ Sct stars, asterisks indicate $\gamma$ Dor stars, and hybrid stars are marked by bullets. The black cross in the right top corner shows typical errors on the values. c) $\left(T_{\text {eff }}, \log g\right)$-diagram of the subsample of 69 Kepler stars for which accurate $T_{\text {eff }}$ and $\log g$ values are available. The colour-codes are the same as for panel b). d) ( $T_{\text {eff }}, \log g$ )-diagram of the subsample of Kepler stars that show pulsations with amplitudes higher than $1000 \mathrm{ppm}(>1 \mathrm{mmag})$. Evolutionary tracks for MS stars with masses $1.4 M_{\odot}, 1.7 M_{\odot}$, and 2.0 $M_{\odot}$ are plotted with grey dotted lines. The evolutionary tracks have been computed using the Code Liégeois d'Évolution Stellaire (CLES, Scuflaire et al. 2008). The input physics included is similar to the one used in Dupret et al. (2005) with the following values for the modelling parameters $\alpha_{\mathrm{MLT}}=1.8, \alpha_{\mathrm{ov}}=0.2$ and $Z=0.02$. The solid thick black and light grey lines mark the blue and red edge of the observed instability strips of $\delta$ Sct and $\gamma$ Dor stars, as described by Rodríguez \& Breger (2001) and Handler \& Shobbrook (2002), respectively. In the on-line version of the paper the symbols representing the $\delta$ Sct, $\gamma$ Dor, and hybrid stars are red, blue, and black, respectively.

eight stars have high $v \sin i$ values, six have moderate values $\left(40<v \sin i<90 \mathrm{~km} \mathrm{~s}^{-1}\right)$, and two low values $\left(v \sin i<40 \mathrm{~km} \mathrm{~s}^{-1}\right)$. Of the 20 hybrid stars almost all have high $v \sin i$ values, with six stars having $v \sin i$ values above $200 \mathrm{~km} \mathrm{~s}^{-1}$. Extrapolating these numbers to the full sample, we expect that many stars in the sample are moderate-to-fast rotators.

\subsubsection{Frequencies and amplitudes}

Up to 500 non-combination frequencies are detected in the Kepler time series of a single star (see Table 3). These large numbers of frequencies are in sharp contrast with the small number of frequencies observed from the ground, e.g. up to 79 pulsation and combination frequencies for the $\delta$ Sct star FG Vir (e.g. Breger et al. 2005) and up to 10 frequencies in the $\gamma$ Dor hybrid candidate HD 49434 (Uytterhoeven et al. 2008), but are commonly seen in space observations because of their higher precision and sensitivity to low-amplitude variations (e.g. Poretti et al. 2009; García Hernández et al. 2009; Chapellier et al. 2011). However, it needs to be carefully checked whether all of the apparent individual frequencies are of pulsational origin.

For the majority of stars (66\%), less than 100 frequencies were found, and $10 \%$ of the stars show variations with more than 200 frequencies. If we look at the extreme cases we find that for 29 stars $(6 \%)$ fewer than 10 frequencies were detected, while for 5 stars $(1 \%)$ more than 400 frequencies were found. The middle panel of Fig. 11 shows the distribution of the number of detected frequencies for the $\delta$ Sct (top, dark grey), hybrid (middle, middle grey), and $\gamma$ Dor (bottom, light grey) stars. The highest number of frequencies are found for hybrid stars. It is worth mentioning that the number of detected frequencies versus $T_{\text {eff }}$ follows a distribution that peaks near $7700 \mathrm{~K}, 7500 \mathrm{~K}$, and $7000 \mathrm{~K}$ for $\delta$ Sct, hybrid, and $\gamma$ Dor stars, respectively. More modes are excited 

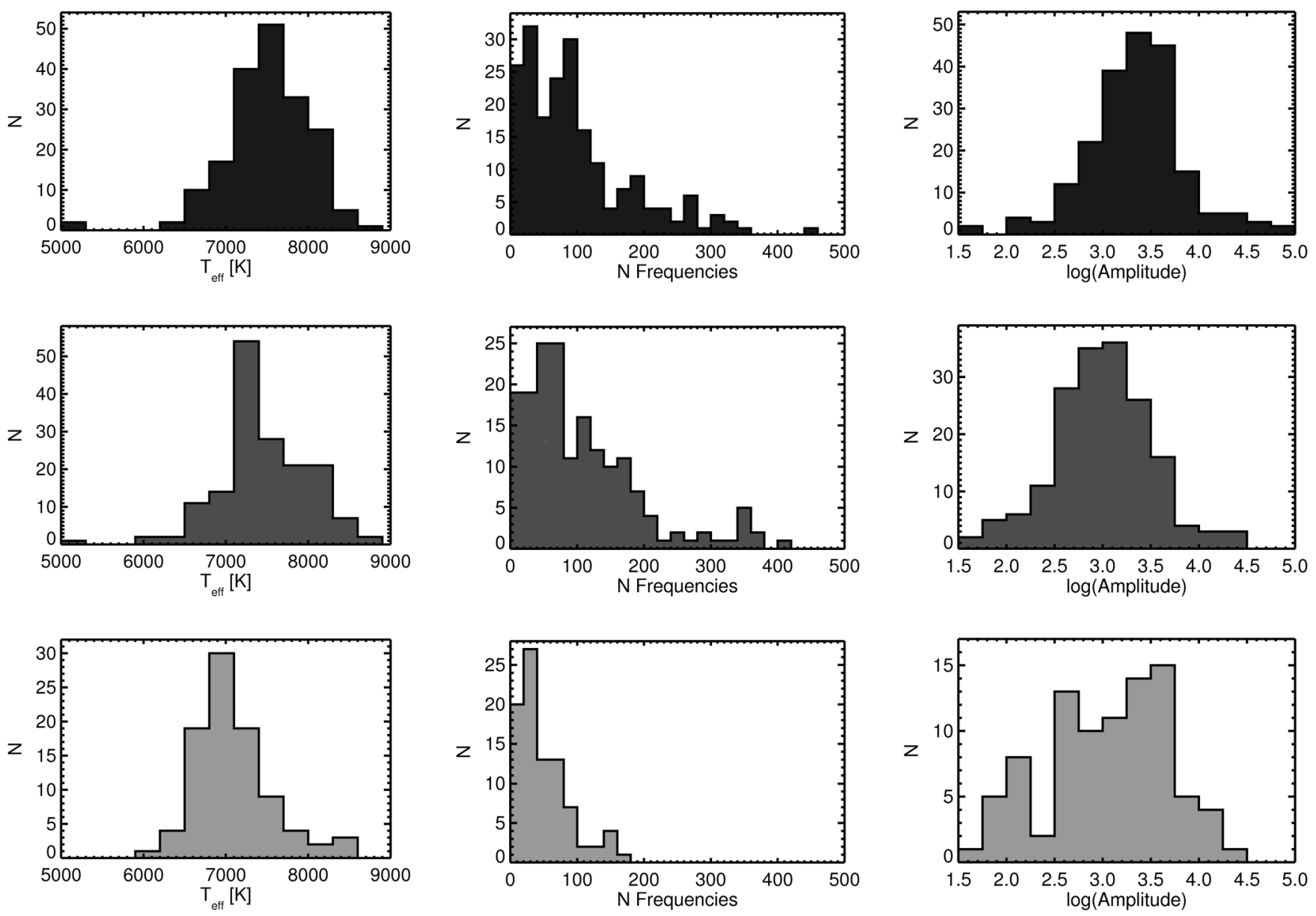

Fig. 11. Distribution in $T_{\text {eff }}$ (left column), number of detected (independent) frequencies (middle column) and highest amplitude (ppm, in logarithmic scale) (right column), for the three groups of A-F type stars: $\delta$ Sct stars (top; dark grey), hybrid stars (middle; middle grey), and $\gamma$ Dor stars (bottom; light grey). The number of stars belonging to each bin $(N)$ is indicated on the $Y$-axis.

near the centre of the $\delta$ Sct instability strip. For the hybrid and $\gamma$ Dor stars most detected frequencies are found towards the red edge of the (overlap in the) instability strip.

The right panel of Fig. 11 shows the distribution of the highest measured amplitude in ppm logarithmic scale ( $\log ($ Amplitude $)$ ) for the different groups using the same colourcode as before. The range in highest amplitude measured is 40 to $155000 \mathrm{ppm}$. For about $59 \%$ of the stars the highest amplitude is lower than $2000 \mathrm{ppm}$. Only 16 stars (3.5\%) show variability with highest amplitudes below 100 ppm, while 26 stars (5\%) have amplitudes above $10000 \mathrm{ppm}$. In general, higher amplitudes are detected in $\delta$ Sct pulsators than in $\gamma$ Dor stars. We point out that the origin of high peaks detected in $\gamma$ Dor stars, e.g. the amplitude of $23000 \mathrm{ppm}$ in the star KIC 7304385, is most likely related to the rotation of the star. It is worth mentioning that amplitudes above $10000 \mathrm{ppm}$ are also detected in faint targets. The highest amplitudes are found for stars within the temperature range $T_{\text {eff }}=6600-7100 \mathrm{~K}$, which is the cool part of the instability strips.

We detected $\delta$ Sct frequencies between 4 and $80 \mathrm{~d}^{-1}$. We found indications that a handful of stars vary with even shorter periods. However, these short periods need to be confirmed by means of a careful investigation of the specific frequency spectra, which is beyond the scope of this paper.

When considering the $\delta$ Sct stars and hybrid classes, which amount to atotal of 375 stars, we find that $56 \%$ shows an upper frequency limit between 40 and $70 \mathrm{~d}^{-1}$. Only $10 \%$ of the $\delta$ Sct and hybrid stars have frequencies up to $80 \mathrm{~d}^{-1}$, and $9 \%$ only show variations with frequencies lower than $20 \mathrm{~d}^{-1}$. We note that $\gamma$ Dor-dominated hybrids that show variations with frequencies higher than $60 \mathrm{~d}^{-1}$ are rare (three stars in our sample).

The majority of the hybrid stars detected in the Kepler data show all kinds of periodicities within the $\gamma$ Dor and $\delta$ Sct range (see Cols. 6 and 7 in Table 3 which give the frequency range of the detected frequencies in the $\gamma$ Dor and $\delta$ Sct domains). This observational fact is interesting because from a theoretical point of view no excited modes are expected between about 5 and $10 \mathrm{~d}^{-1}$, i.e. the so-called "frequency gap" (see, e.g. Grigahcène et al. 2010). Only for five hybrid stars a "frequency gap" is observed $^{7}$. Possible explanations for the absence of gaps, within the present non-adiabatic theories, are that the frequencies within the gap are high-degree and/or rotationally split modes (Bouabid et al. 2009).

\section{A first step towards understanding the relation between $\delta$ Sct, $\gamma$ Dor, and hybrid stars}

As presented in the previous section, it is not trivial to distinguish between the three groups of variable A-F type stars defined in Sect. 6.1. The relation between the three groups is currently unclear as well because $\delta$ Sct, $\gamma$ Dor, and hybrid stars

7 The six hybrid stars that show a "frequency gap" are: KIC 3851151 , KIC 4556345, KIC 7770282, KIC 9052363, and KIC 9775454. 
coincide in the ( $\left.T_{\text {eff }}, \log g\right)$-diagram (Fig. 10). Driven by the idea to find observables based on physical concepts that allow insight in the different internal physics of the three types of stars, we constructed two new observables that can provide an alternative way to improve our understanding of the relation between the three groups. We point out that several observational parameters can be found that reflect the different inherent properties of the three groups in one way or another. For instance, $\delta$ Sct stars pulsate with shorter periods, and are generally hotter than $\gamma$ Dor stars. A combination of these parameters will lead to a differentiation of the groups, such as for instance a $\left(T_{\text {eff }}, f_{\max }\right)$-diagram, with $f_{\max }$ the frequency associated to the highest amplitude mode. However, we emphasize that our aim is to find observables that can be directly related to the internal physics of the stars.

According to the current instability theories, which need to be revised following the results presented in this work, the main driving process of the oscillations in $\delta$ Sct stars is related to the opacity variations in the ionization zones (Unno et al. 1989). These zones are located in the region where the main energy transport mechanism is convection and where a small quantity of energy is transported by radiation. The total amount of driving energy going into the mode is directly related to the radiative luminosity in this zone, and this latter quantity is a function of the convective efficiency. Therefore, we expect a relation between the energy of the observed modes and the convective efficiency of the outer convective zone. We searched for this relation and constructed two observables, energy and efficiency, that are estimates of the energy and the convective efficiency, using the available observational data.

\subsection{Energy}

The kinetic energy of a wave is given by

$E_{\mathrm{kin}}=\frac{1}{2} f\left(\rho_{*}\right)(A \zeta)^{2}$,

where $f$ is a function of the stellar density $\rho_{*}, A$ is the amplitude of the oscillation, and $\zeta$ is the pulsation frequency. Using the available observational data, we construct the following observable that we call energy, which is a first approximation and estimate of the kinetic energy of the wave:

energy $\equiv\left(A_{\max } \zeta_{\max }\right)^{2}$,

where $A_{\max }$ and $\zeta_{\max }$ refer to the highest amplitude mode of the star (in ppm), and associated frequency (in $\mathrm{d}^{-1}$ ). The pulsation amplitude is a function of the observed amplitude and the relative variation of the flux, and is given by the expression (Moya \& Rodríguez-López 2010):

$\Delta R / R=-\frac{\Delta m}{\ln (5+10 \mathrm{~d} T)}$,

where $\frac{\Delta R}{R}$ is the relative pulsational amplitude, $\Delta m$ the observed magnitude variation of the mode, and $\mathrm{d} T$ is given by

$\mathrm{d} T=\frac{\delta T_{\mathrm{eff}}}{T_{\mathrm{eff}}} / \frac{\xi_{r}}{r},(r=R)$,

with $\xi_{r}$ the variation in radius of the mode, and $\mathrm{d} T$ is evaluated at the surface of the $\operatorname{star}(r=R)$.

Non-adiabatic calculations of a representative model of a hybrid pulsating A-F type star including time dependent convection (Grigahcène et al. 2005) show that the difference between
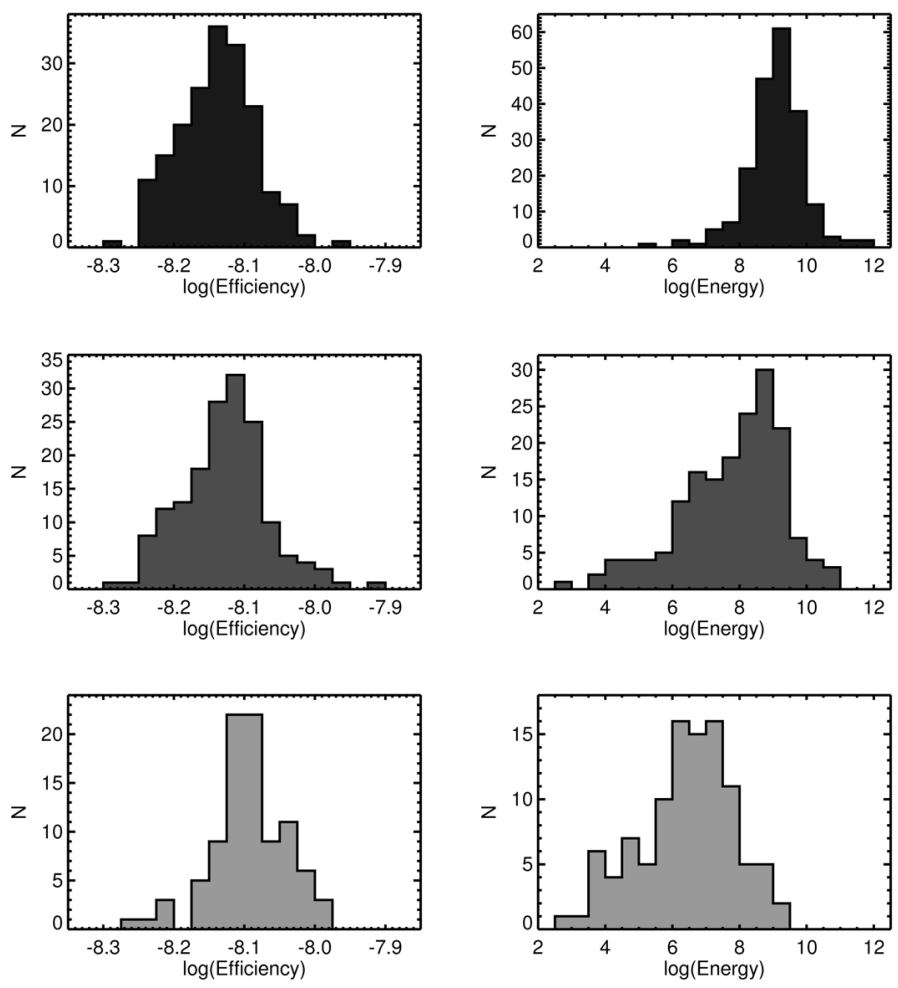

Fig. 12. Distribution in $\log ($ energy) (right), and $\log ($ efficiency) (left) for the $\delta$ Sct (top, dark grey), hybrid (middle, middle grey), and $\gamma$ Dor (bottom, light grey) stars. The number of stars belonging to each bin $(N)$ is indicated on the $Y$-axis.

the predicted $\mathrm{d} T$ value of asymptotic g-modes ( $\gamma$ Dor stars) and low-order p-modes ( $\delta$ Sct stars) is around one order of magnitude or less, where the $\delta$ Sct stars have higher values. Therefore, we can directly use the observed magnitude variation as a measurement of the radial amplitude variation. That we are using an approximation does not change the conclusions of the present study, because the observed differences are larger than two orders of magnitude (see Figs. 12 and 13).

The right column of Fig. 12 shows the distribution in $\log ($ energy) for the $\delta$ Sct (top, dark grey), hybrid (middle, middle grey), and $\gamma$ Dor (bottom, light grey) stars. Clearly, the weight of the distribution is located in the region $\log ($ energy $)>8$ for stars dominated by frequencies in the $\delta$ Sct domain, and in the region $\log ($ energy $)<8$ for stars with dominant $\gamma$ Dor pulsations.

\subsection{Efficiency}

In the introduction of this section we pointed out that a relation between the convective efficiency and mode excitation can exist. Recent studies on convective efficiency of the outer convective zone of F-G-K stars using 3D models show that the convective efficiency is related to the position of the star in the HertzsprungRussell (HR-) diagram (Trampedach \& Stein 2011). To construct an observable related to the convective efficiency that can be described with only variables related with the position in the HR-diagram, we found inspiration in the analytic description of the convective energy given by the mixing length theory ${ }^{8}$

8 In analogy to the mean free parameter in gas kinetic theory, the mixing length is defined as the mean distance over which a fluid bulb conserves its properties. Generally, the mixing-length is assumed to be proportional to the pressure-scale height by a factor $\alpha$ that is usually called mixing-length parameter. 

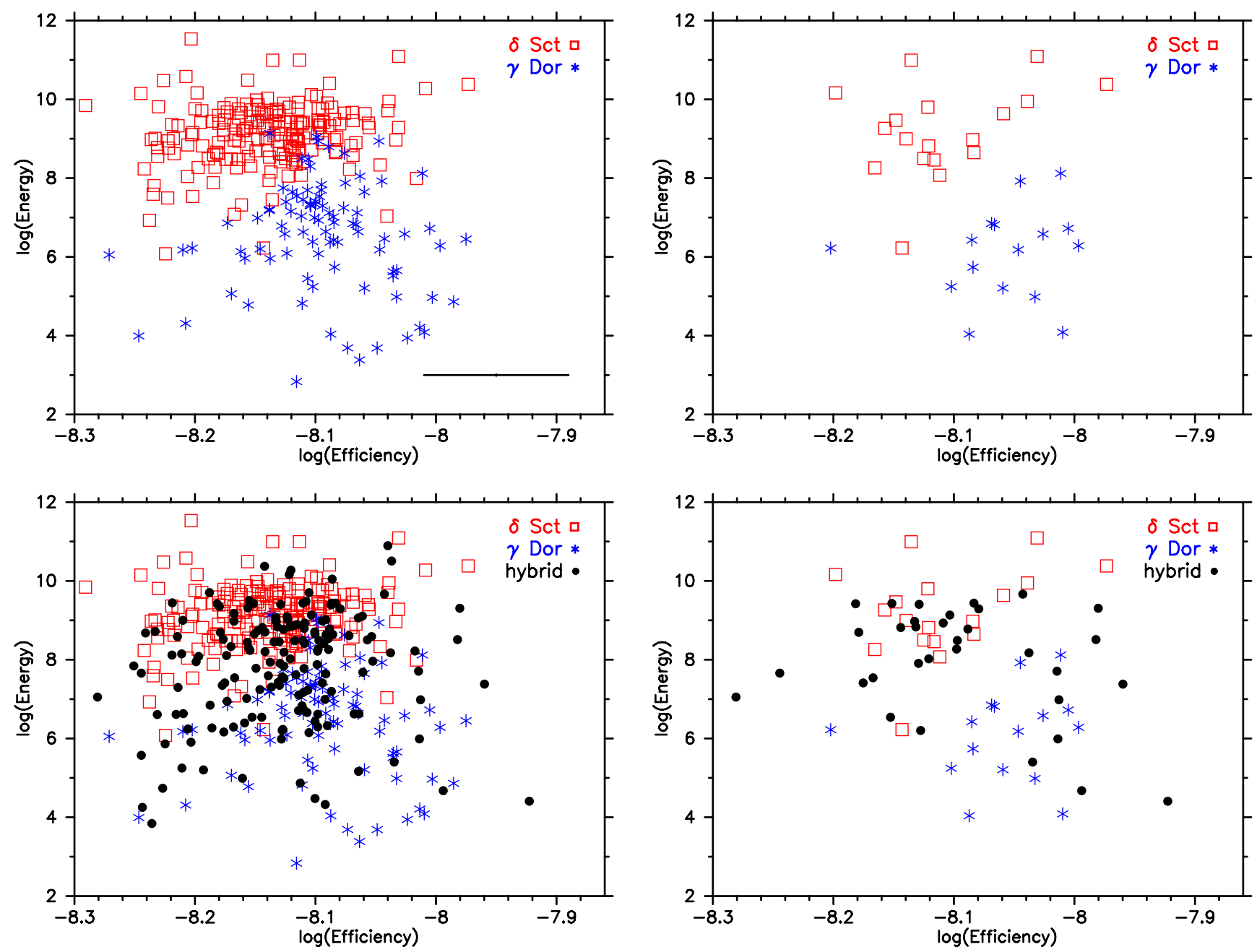

Fig. 13. Observable $\log$ (energy) plotted versus $\log ($ efficiency) for $\delta$ Sct (open squares) and $\gamma$ Dor (asterisks) stars only (top), and for hybrid stars as well (bullets) (bottom). The left panels include all 480 Kepler stars that are assigned to one of the three groups. The right panels show the 49 stars for which reliable values of $T_{\text {eff }}$ and $\log g$ are available. In the on-line version of the paper the symbols representing the $\delta$ Sct, $\gamma$ Dor and hybrid stars are red, blue, and black, respectively. The cross in the right bottom corner of the top left panel represents the typical error bars on the values: 0.04 dex and 0.12 dex for $\log ($ energy $)$ and $\log ($ efficiency), respectively.

(Böhm-Vitense 1958). There, the convective efficiency, $\Gamma$, is defined as

$\Gamma=\left[\frac{A^{2}}{a_{0}}\left(\nabla_{\mathrm{rad}}-\nabla\right)\right]^{1 / 3}$,

with $a_{0}$ a constant, $\nabla_{\text {rad }}$ and $\nabla$ the radiative and real temperature gradient, respectively, and

$A \sim \frac{c_{p} \kappa p \rho c_{\mathrm{s}} \alpha^{2}}{9 \sigma T^{3} g \sqrt{2 \Gamma_{1}}}$

(see Cox \& Giuli 1968).

This quantity, which measures the ratio between the convective and radiative conductivity, depends on a large number of physical variables: the specific heat capacity at constant pressure $c_{p}$, the opacity $\kappa$, the pressure $p$, the stellar density $\rho$, the sound velocity $c_{\mathrm{s}}$, the mixing length parameter $\alpha$, the StephanBoltzmann constant $\sigma$, the temperature $T$, the gravity $g$, and the first adiabatic coefficient $\Gamma_{1}$. Because we only have information on a limited number of observational variables, our estimate of the quantity is only an approximation. Inspired by these equations, we searched for the combination of temperature and gravity that empirically provided the best means to separate between $\gamma$ Dor and $\delta$ Sct stars (see statistical test below), and define the observable efficiency as

efficiency $\equiv\left(T_{\mathrm{eff}}^{3} \log g\right)^{-2 / 3} \sim \Gamma$.

Because the efficiency of the convective zone is expected to be higher for $\gamma$ Dor stars than for $\delta$ Sct stars, the observable efficiency should have a higher value for $\gamma$ Dor stars than $\delta$ Sct stars. This behaviour is indeed observed, as illustrated in the left panel of Fig. 12, where the distribution in $\log$ (efficiency) is given. The majority of $\delta$ Sct stars have values $\log ($ efficiency $)<-8.1$ dex, while the histograms for $\gamma$ Dor pulsators peak in the region $\log ($ efficiency $)>-8.1$ dex.

\subsection{Efficiency versus energy}

When we plot the two new observables, $\log ($ energy) versus $\log ($ efficiency), the groups of $\delta$ Sct and $\gamma$ Dor stars are fairly well separated (see top panels Fig. 13). A $\log ($ energy) value 
of 8 leaves $90 \%$ of the $\delta$ Sct and $\gamma$ Dor stars separated. The bottom panel of Fig. 13 shows the same diagram with values for the hybrid stars included, using the same colours and symbols as before. Typical errors on the values are 0.04 dex and 0.12 dex for $\log ($ energy) and $\log ($ efficiency), respectively. The hybrid stars are placed in the intermediate region. We observed that $\delta$ Sct ( $\gamma$ Dor) dominated hybrids fall in the same region as the $\delta$ Sct ( $\gamma$ Dor) stars.

We performed a Mann-Whitney $U$ test with an adapted $p$-value ( $p=0.0166$ ) according to the closed-test principle described in Horn \& Vollandt (1995), to statistically investigate if the mean of the distribution in $\log ($ energy) and $\log ($ efficiency) is different for the three different groups. The test shows that the difference in the mean of the distributions in both $\log ($ energy) and $\log ($ efficiency) is statistically significant for all groups. However, the apparent separation in $\log ($ efficiency) becomes less evident when we take the considerable error bars into account. We also performed a $\chi^{2}$ test (as described by Press et al. 1992) to determine if the distributions themselves were different. All distributions are statistically significant, save for the $\gamma$ Dor versus hybrid star efficiencies, where they are marginally similar. This conclusion holds even if we vary the $T_{\text {eff }}$ and $\log g$ values within the error ranges and recompute the efficiencies or vary the inputs into the energies. We point out once again that the definition of efficiency is only a rough estimate of the theoretical expression for the convective efficiency, and might - at this stage - not be refined enough to display the separating power between the groups we expect the convective efficiency to have. In a followup investigation we will assess the goodness of approximation of our definition of efficiency by comparison with values of the convective efficiency as given by Eq. (6), calculated for several model stars, and finetune its definition.

The two new approximate observables energy and efficiency reflect the different internal physics of oscillators with dominant $\delta$ Sct pulsations and oscillators dominated by $\gamma$ Dor pulsations, and seem to allow us to distinguish between them. However, it needs to be further investigated if the two observables can be considered as independent parameters. This, together with an exploration of the physical mechanisms behind the instability of these stars, is the topic of a forthcoming paper. The observables energy and efficiency are promising starting points to explore the relation between $\delta$ Sct, $\gamma$ Dor and hybrid stars, but need to be refined.

\section{Summary, discussion, and future prospects}

We analysed the Kepler light curves based on survey phase data with time spans between $9 \mathrm{~d}$ and $322 \mathrm{~d}$ available through KASOC and associated frequency spectra of 750 candidate A-F type stars in search for $\delta$ Sct, $\gamma$ Dor, and hybrid pulsators. The main results are:

- The Kepler light curves of the sample of 750 candidate A-F type stars show a variety in variability behaviour.

- Observationally, we propose three main groups to describe the observed variety: $\gamma$ Dor, $\delta$ Sct, and hybrid stars. The latter group includes both $\delta$ Sct-dominated and $\gamma$ Dor-dominated hybrid stars. About $63 \%$ of the sample are unambiguously assigned to one of the three groups.

- About $23 \%$ of the sample are hybrid candidates (171 stars, or $36 \%$ of the stars assigned to the three groups). This is in strong contrast with the number of hybrid candidates so far observed from the ground, but compatible with the first Kepler study of $\gamma$ Dor and $\delta$ Sct variables by
Grigahcène et al. (2010). The far superior precision of the Kepler space data opens a new window in detecting lowamplitude variations. Kepler will be ideal to study hybrid behaviour in different types of stars, such as roAp stars (Balona et al. 2011a), sdB stars (Østensen et al. 2010), and B stars (Balona et al. 2011b).

- We presented a characterization of the stars in terms of number of detected frequencies, frequency range, and typical pulsation amplitudes, which provides valuable feedback for models and instability studies. This is the first time that this kind of information is available for a substantial sample of stars. Up to 500 non-combination frequencies are detected in the Kepler time series of a single star. The highest pulsation amplitude measured is $58000 \mathrm{ppm}$. The shortest detected $\delta$ Sct periods are about $18 \mathrm{~min}$. We find that hybrid stars show all kinds of periodicities within the $\gamma$ Dor and $\delta$ Sct range. In particular, the majority of hybrid stars shows frequencies between 5 and $10 \mathrm{~d}^{-1}$. From a theoretical point of view, this result presents a number of challenges, because the currently accepted over-stability mechanisms cannot explain the presence of pulsational modes in the wide frequency ranges observed with Kepler. It needs to be investigated if and to what extent the presence of stochastic modes, highdegree, and/or rotationally split modes with high amplitudes, granulation and effects of convection can explain part of the unexpected observed modes.

- The location of $\gamma$ Dor and $\delta$ Sct classes in the $\left(T_{\text {eff }}, \log g\right)$ diagram has been extended (Fig. 10). We find indications that Kepler $\delta$ Sct stars exist beyond the red edge of the observational instability strip, while Kepler $\gamma$ Dor pulsations seem to appear in both hotter and cooler stars than observed so far. The Kepler hybrid stars occupy the entire region between the blue edge of the $\delta$ Sct instability strip and the red edge of the $\gamma$ Dor instability strip and beyond. These results, if confirmed by verification of the temperature and $\log g$ values in a more comprehensive sample, imply that the observational instability strips need to be extended to accommodate the Kepler $\delta$ Sct and $\gamma$ Dor stars. From a theoretical point of view, the overall presence of hybrid stars implies an investigation of other pulsation mechanisms to supplement the $\kappa$ mechanism and convective blocking effect to drive hybrid pulsations.

- Two new "observables" that reflect the different internal physics of $\delta$ Sct and $\gamma$ Dor pulsators are introduced to investigate the relation between the two types of pulsations (Fig. 13): (1) efficiency, related to the convective efficiency of the outer convective zone, and a function of $T_{\text {eff }}$ and $\log g$; and (2) energy, the driving energy of a mode, and a function of the highest observed frequency amplitude and the associated frequency. Both observables are empirical and are constructed using only available measured variables. The impact and physical significance of the group separation in the $(\log ($ efficiency $), \log ($ energy $))$-diagram needs to be investigated in more detail. The two new observables are a promising starting point for further investigations of the relation between $\delta$ Sct, $\gamma$ Dor and hybrid stars.

- Our study indicates that $\mathrm{Kp}=14$ mag is a cut-off magnitude for detection of variations with amplitudes below $20 \mathrm{ppm}$ in A-F type stars with Kepler.

- Sixteen percent of the sample stars show no clear variability within the expected range of frequencies for $\delta$ Sct and $\gamma$ Dor stars. Faint and cool stars predominate this sample. Among the stars, we identified 75 candidate solar-like stars. No correlation between non-variability and the length of the 
available dataset or the available cadence mode is found. We find indications for the presence of constant stars inside the instability strips of A-F type pulsators.

- The remaining $21 \%$ of sample stars are identified as a Cepheid, B-type stars, red giant stars, stars that show stellar activity, or binaries. At least $12 \%$ of the sample are identified as a binary or multiple system, based on investigation of the Kepler light curve or on input from the literature. Many long-period binaries are expected to be among the remaining stars of the sample. 3.5\% of the sample stars shows eclipses. Several of the EBs have variable components, including $\delta$ Sct, $\gamma$ Dor, and hybrid stars.

Clearly, space missions are changing the landscape of $\gamma$ Dor and $\delta$ Sct pulsators. We aimed at a global analysis of the sample stars. A careful seismic analysis of individual stars is needed to confirm their classification, clarify the observed variety in pulsational behaviour, fully characterize the properties of the $\delta$ Sct, $\gamma$ Dor, and hybrid groups, understand their relationship, clarify the driving mechanism(s) for each group, and elaborate on the variables energy and efficiency. The observational results with Kepler presented here open up several new questions and theoretical challenges for the current models related to pulsational instability, thermodynamics, and stellar structure. We mention here some topics for further investigation.

To be able to place the stars confidently in the $\left(T_{\text {eff }}, \log g\right)$ diagram, estimate the projected rotational velocity, and derive accurate abundances, at least one high-resolution spectrum is needed for each star. To this end, an observational campaign is ongoing (Uytterhoeven et al. 2010a,b). Most stars of the $\delta$ Sct, $\gamma$ Dor, and hybrid stars in our sample with magnitude $\mathrm{Kp} \leq 10.5$ mag have recently been observed or are scheduled to be observed in the coming months. However, $70 \%$ of the stars in Table 3 are fainter than magnitude $\mathrm{Kp}=10.5 \mathrm{mag}$, for which it is time-consuming and less practical to observe them with the available 2-m class telescopes that are equipped with a highresolution spectrograph.

Because the oscillation modes in A-F type stars do not produce evident frequency patterns in their mode spectra, as is the case for solar-like oscillators, the identification of pulsation modes benefits from high-resolution spectral or multi-colour time series. Here we encounter limitations owing to the relative faintness of the Kepler sample too. For instance, it is only feasible to efficiently spectroscopically monitor the few brightest (Kp $\leq 9 \mathrm{mag}$ ) stars from the ground, while multi-colour photometry can go a few magnitudes fainter. Moreover, it will be impossible with the current instrumentation to detect the pulsation amplitudes of the order of a few $\mu$ mag from the ground. Therefore, only for a limited selection of the stars in Table 3, i.e. bright stars exhibiting high-amplitude variations, will it be feasible to organize ground-based follow-up campaigns.

For all other stars, we will have to rely on extracting information on the pulsation modes directly from frequency patterns observed in the Kepler data. Quasi-periodic patterns have been observed before in $\delta$ Sct stars (Handler et al. 1997; García Hernández et al. 2009). But in fast rotating stars the rotation destroys regular frequency and period patterns of p- and g-modes, which complicates the mode identification (e.g. Lignières et al. 2006; Ballot et al. 2010). For slowly rotating g-mode pulsators $\left(V_{\text {rot }}<70 \mathrm{~km} \mathrm{~s}^{-1}\right.$ ), a mode-identification technique has been developed that relies only on accurate values of at least three frequencies (Frequency Ratio Method, Moya et al. 2005; Suárez et al. 2005), which is ideal to apply to the information extracted from the Kepler white light, without colour or spectral information. Unfortunately, many of our stars are moderate-to-fast rotators (see Sect. 7.2). Hence, the mode identification will be very challenging and will require more investigation.

An individual analysis of the candidate hybrid stars is needed to confirm their hybrid status and to firmly characterize their pulsation properties. The current theoretical instability models for hybrid stars need to be revised to be able to accommodate all stars that have been proposed as hybrid candidates in this paper. This includes a revision of the mechanisms that allow driving of $\mathrm{p}$ - and g-modes in A-F type stars with a broad range of temperatures. Additional processes that can be investigated with possible effect on the driving are stochastic excitation (Houdek et al. 1999; Samadi et al. 2002), a convective driving mechanism similar to g-mode pulsations in white dwarfs (Goldreich \& Wu 1999), a $\kappa$ mechanism-related effect presented by Gautschy \& Löffler (1996) and Löffler (2000), and radiative levitation (Turcotte et al. 2000). Asteroseismic diagnostics have been studied to find signatures of stochastic mechanisms at the origin of the instability of $\gamma$ Dor oscillators (Pereira et al. 2007). In that work, this possibility was not discarded, but continuous and precise space data were not yet available. The Kepler time series of the sample of stars studied here will be an ideal new testbed for this method.

The long, continuous time series that Kepler will deliver during its lifetime will unveil a large number of amplitudes at $\mu$ mag level. This precision will open up opportunities to search for signatures of granulation in the variable star light (Kallinger \& Matthews 2010). Spectroscopically, convective signatures have been detected in the microturbulence and line broadening of A-F type stars cooler than $T_{\text {eff }}=10000 \mathrm{~K}$ (Landstreet et al. 2009).

Also the theoretical instability strips of the $\gamma$ Dor and $\delta$ Sct pulsators need revision. As shown in Fig. 1, stars exhibiting purely $\gamma$ Dor or $\delta$ Sct pulsations seem to exist beyond the current blue and red edge of the respective instability strips. Moreover, it is worth investigating if the evolutionary phase of $\gamma$ Dor stars can be derived from properties in their frequency spectra, as is recently suggested by Bouabid et al. (2011), based on a theoretical study of seismic properties of MS and pre-MS $\gamma$ Dor pulsators.

Another open question is the existence of non-variable A-F type stars inside the instability strips. So far, it is suggested (Poretti et al. 2003; Breger 2004) that all seemingly constant stars in the instability strip are low-amplitude pulsators. In this study we find indications that non-variability exists within the instability strip, but a more in-depth investigation based on a more comprehensive sample of stars with precise values of $T_{\mathrm{eff}}$ and $\log g$ is needed to confirm this.

Furthermore, candidate $\gamma$ Dor stars with only a few excited dominant modes deserve to be looked at in more detail. The relation between rotation and pulsations is not yet clear. Moreover, the differentiation between pulsations and rotational variability proves to be very difficult (Breger 2011; Monnier et al. 2010). In the pilot study by Balona et al. (2011d) it was suggested that pulsation and rotation periods might be very closely related. It needs to be investigated to which extent the rotation influences the excitation of the observed modes. To help this investigation, $v \sin i$ values are needed.

Constraints on important physical parameters that are crucial for seismic modelling, such as stellar radius and mass, can be derived directly for pulsators in binary systems (e.g. Tango et al. 2006; Desmet et al. 2010). Our sample consists of several binaries and eclipsing systems with (a) pulsating component(s) (see Table 4). Hence, these targets in particular are very 
promising for dedicated ground-based follow-up observations, and a seismic analysis. Moreover, it will be interesting to investigate the effect of tidal interactions on pulsation frequencies (e.g. Uytterhoeven et al. 2004; Derekas et al. 2011).

Four of the EBs with a candidate $\gamma$ Dor, $\delta$ Sct, or hybrid component in our sample are known as chemically peculiar stars (see Table 1). Three candidate hybrid stars (out of 61 stars with known spectral type), four candidate $\delta$ Sct stars (out of 67 stars), and one candidate $\gamma$ Dor star (out of 25 stars) are also Ap or Am stars. So far, we detected both p- and g-mode pulsators among the chemically peculiar stars. Balona et al. (2011c) stated that the instability strip of pulsating Am type stars and $\delta$ Sct stars do not differ much. With the current small number statistics, it is not clear whether Ap/Am stars are indeed rare among $\gamma$ Dor stars (Handler \& Shobbrook 2002). One of the open questions is if chemical peculiarity is related to hybridity. The first discovered hybrid HD 8801 (Henry \& Fekel 2005) intruigingly turned out to be an Am star. In a recent abundance study by Hareter et al. (2011) one of the two studied hybrid stars is also confirmed as being a chemically peculiar star. Together with the results of this study, this brings the total of known chemically peculiar hybrid stars to five. There is currently no evidence for a direct link between chemical peculiarity and hybrid behaviour, but a careful abundance analysis of a representative sample of hybrid stars is needed to confirm this.

Many more (candidate) $\delta$ Sct, $\gamma$ Dor, and hybrid stars are expected to be among the stars observed by Kepler. Debosscher et al. (2011) reported the discovery of many additional $\delta$ Sct and $\gamma$ Dor candidates in the public Kepler Q1 data. Also, a considerable fraction of the host stars of the recently published 1235 Kepler planet candidates (Borucki et al. 2011) turn out to be A-F type stars. Hence, we have promising prospects in studying and understanding the A-F type star variable behaviour in detail through a much larger and more complete sample of A-F stars in the Kepler field when longer timestrings of Kepler data will become publicly available. Kepler is definitely opening the window towards the accurate characterization of pulsating A-F stars.

Acknowledgements. We are grateful to Joanna Molenda-Żakowicz, James Nemec and the anonymous referee for their suggestions and comments to improve this paper. Funding for the Kepler mission is provided by NASA's Science Mission Directorate. We thank the entire Kepler team for the development and operations of this outstanding mission. K.U. acknowledges financial support by the Deutsche Forschungsgemeinschaft (DFG) in the framework of project UY 52/1-1, and by the Spanish National Plan of R\&D for 2010, project AYA2010-17803. A.M. acknowledges the funding of AstroMadrid (CAM S2009/ESP-1496). E.N. and A.P. acknowledge the financial support of the NN 203405139 and NN 203302635 grant, respectively, from the MNiSW. The work by G.H. and V.A. was supported by the Austrian Fonds zur Förderung der wissenschaftlichen Forschung under grant P20526-N16. L.F.M. acknowledge financial support from the UNAM under grant PAPIIT IN114309. R.Sz. and L.L.K. have been supported by the "Lendület" program of the Hungarian Academy of Sciences and the Hungarian OTKA grants K83790 and MB08C 81013. RSz was supported by the János Bolyai Research Scholarship of the Hungarian Academy of Sciences. SH acknowledges financial support from the Netherlands Organisation for Scientific Research (NWO). This research has been funded by the Spanish grants ESP2007-65475-C02-02, AYA 2010-21161C02-02 and CSD2006-00070. The research leading to these results has received funding from the European Community's Seventh Framework Programme (FP7/2007-2013) under grant agreement No. 269194. This research has made use of the SIMBAD database, operated at CDS, Strasbourg, France, and is partly based on observations obtained at the Observatorio Astrónomico Nacional-San Pedro Mártir (OAN-SPM), Baja California, Mexico, at the Observatoire de Haute Provence, France, and at the Thüringer Landessternwarte Tautenburg, Germany. We acknowledge with thanks the variable star observations from the AAVSO International Database contributed by observers worldwide and used in this research.

\section{References}

Abt, H. A. 1984, ApJ, 285, 247

Abt, H. A. 2004, ApJS, 155, 175

Abt, H. A., \& Cardona, O. 1984, ApJ, 285, 190

Aerts, C. 2001, ApJ, 553, 814

Aerts, C., Eyer, L. \& Kestens, E. 1998, A\&A, 337, 790

Allende Prieto, C., \& Lambert, D. L. 1999, A\&A, 352, 555

Antoci, V., Handler, G., Campante, T. L., et al. 2011, Nature, accepted

Baglin, A., Auvergne, M., Barge, P., et al. 2006, in The CoRoT Mission, Pre-

Launch Status, Stellar Seismology and Planet Finding, ed. M. Fridlund, A.

Baglin, J. Lochard, \& L. Conroy, ESA Publications Division, Nordwijk,

Netherlands, ESA SP-1306, 33

Ballot, J., Lignières, F., Reese, D. R., \& Rieutord, M. 2010, A\&A, 518, A30

Balona, L. A., Krisciunas, K., \& Cousins, A. W. J. 1994, MNRAS, 270, 905

Balona, L. A., Cunha, M. S., Kurtz, D. W., et al. 2011a, MNRAS, 410, 517

Balona, L. A., Pigulski, A., De Cat, P., et al. 2011b, MNRAS, 413, 2403

Balona, L. A., Ripepi, V., Catanzaro, G., et al. 2011c, MNRAS, 414, 792

Balona, L. A., Guzik, J., Uytterhoeven, K., et al. 2011d, MNRAS, in press

Benkő, J. M., Kolenberg, K., Szabó, R., et al. 2010, MNRAS, 409, 1585

Bidelman, W. P., Ratcliff, S. J., \& Svolopoulos, S. 1988, PASP, 100, 828

Böhm-Vitense, E. 1958, Zeitschrift für Astrophysik, 46, 108

Borucki, W. J., Koch, D. G., Brown, T. M., et al. 2010, Science, 327, 977

Borucki, W. J., Koch, D. G., Basri, G., et al. 2011, ApJ, 728, 117

Bouabid, M.-P., Montalbán, J., Miglio, A., et al. 2009, in Stellar Pulsation: Challenges for Theory and Observation, ed. J. A. Guzik, P. A. Bradley (Melville, NY: AIP), AIP Conf. Proc., 1170, 477

Bouabid, M.-P., Montalbán, J., Miglio, A., et al. 2011, A\&A, 531, A145

Breger, M. 2000, in Delta Scuti and Related Stars, ed. M. Breger, \& M. H. Montgomery, ASP Conf. Ser., 210, 3

Breger, M. 2004, in The A-Star Puzzle, ed. J. Zverko, J. Žižnovský, S. J. Adelman, \& W. W. Weiss (Cambridge University Press), Proc. IAU Symp. 224,335

Breger, M. 2011, Carnegie Observatories Astrophysics Series, 5, in press [arXiv: 1104.4392]

Breger, M., Handler, G., Garrido, R., et al. 1997, A\&A, 324, 566

Breger, M., Lenz, P., Antoci, V., et al. 2005, A\&A, 435, 955

Breger, M., Balona, L., Lenz, P., et al. 2011, MNRAS, 414, 1721

Brown, T. M., Latham, D. W., Everett, M. E., \& Esquerdo, G. A. 2011, AJ, in press [arXiv: 1102.0342]

Bruntt, H., De Cat, P., \& Aerts, C. 2008, A\&A, 478, 487

Cannon, A. J. 1925, Ann. Astron. Obs. Harvard Coll., 100, 17

Carrier, F., North, P., Udry, S., \& Babel, J. 2002, A\&A, 394, 151

Catanzaro, G., Frasca, A., Molenda-Żakowicz, J., \& Marilli, E. 2010, A\&A, 517, A3

Catanzaro, G., Ripepi, V., Bernabei, S., et al. 2011, MNRAS, 411, 1167

Chapellier, E., Rodríguez, E., Auvergne, M., et al. 2011, A\&A, 525, A23

Cox, J. P., \& Giuli, R. T. 1968, Principles of stellar structure (New York: Gordon and Breach)

Cuypers, J., Aerts, C., De Cat, P., et al. 2009, A\&A, 499, 967

Debosscher, J., Sarro, L. M., López, M., et al. 2009, A\&A, 506, 519

Debosscher, J., Blomme, J., Aerts, C., \& De Ridder, J. 2011, A\&A, 529, A89

Derekas, A., Kiss, L. L., Borkovits, T., et al. 2011, Science, 332, 216

De Cat, P., Eyer, L., Cuypers, J., et al. 2006, A\&A, 449, 281

Desmet, M., Frémat, Y., Baudin, F., et al. 2010, MNRAS, 401, 418

Dupret, M.-A., Grigahcène, A., Garrido, R., Gabriel, M., \& Scuflaire, R. 2004, A\&A, 414, L17

Dupret, M.-A., Grigahcène, A., Garrido, R., Gabriel, M., \& Scuflaire, R. 2005, A\&A, 435, 927

Eyer, L., \& Aerts, C. 2000, A\&A, 361, 201

Floquet, M. 1970, A\&AS, 1, 1

Floquet, M. 1975, A\&AS, 21, 25

García, R. A., Hekker, S., Stello, D., et al. 2011, MNRAS, 414, 6

García Hernández, A., Moya, A., Michel, E., et al. 2009, A\&A, 506, 79

Gautschy, A., \& Löffler, W. 1996, DSSN, 10, 13

Gilliland, R. L., Brown, T. M., Christensen-Dalsgaard, J., et al. 2010a, PASP, 122,131

Gilliland, R. L., Jenkins, J. M., Borucki, W. J., et al. 2010b, ApJ, 713, 160

Glebocki, R., \& Stawikowski, A. 2000, AcA, 50, 509

Goldreich, P., \& Wu, Y. 1999, ApJ, 511, 904

Grenier, S., Baylac, M.-O., Rolland, L., et al. 1999, A\&AS, 137, 451

Grigahcène, A., Dupret, M.-A., Gabriel, M., Garrido, R., \& Scuflaire, R. 2005, A\&A, 434, 1055

Grigahcène, A., Antoci, V., Balona, L., et al. 2010, ApJ, 713, L192

Gray, R. O., \& Kaye, A. B. 1999, AJ, 118, 2993

Guetter, H. H. 1968, PASP, 80, 197

Guinan, E. F., Bochanski, J. J., Depasquale, J. M., Ribas, I., \& McCook, G. P. 2001, IAU Inform. Bull. Var. Stars, 5062, 1 
Guzik, J. A., Kaye, A. B., Bradley, P. A., Cox, A. N., \& Neuforge, C. 2000, AJ, 542,57

Handler, G. 1999, MNRAS, 309, L19

Handler, G. 2009a, AIPC, 1170, 403

Handler, G. 2009b, MNRAS, 398, 1339

Handler, G., \& Shobbrook, R. R. 2002, MNRAS, 333, 251

Handler, G., Pikall, H., O’Donoghue, D., et al. 1997, MNRAS, 286, 303

Hareter, M., Reegen, P., Miglio, A., et al. 2010, AN, P49 [arXiv: 1007 . 3176]

Hareter, M., Fossati, L., Weiss, W., et al. 2011, ApJ, submitted

Hartman, J. D., Bakos, G., Stanek, K. Z., \& Noyes, R. W. 2004, AJ, 128, 1761

Hauck, B., \& Mermilliod, M. 1998, A\&AS, 129, 431

Henry, G. W., \& Fekel, F. C. 2005, AJ, 129, 2026

Henry, G. W., Fekel, F. C., Kaye, A. B., \& Kaul, A. 2001, AJ, 122, 3383

Henry, G. W., Fekel, F. C., \& Henry, S. M. 2005, AJ, 129, 2815

Henry, G. W., Fekel, F. C., \& Henry, S. M. 2011, AJ, 142, 39

Hill, P. W., \& Lynas-Gray, A. E. 1977, MNRAS, 180, 691

Hill, S. J., \& Schilt, J. 1952, Contributions from the Rutherford Observatory of Columbia University New York, 32, 1

Hoffleit, D. 1951, Harvard College Observatory Bulletin, 920, 32

Horn, M., \& Vollandt, R. 1995, in Multiple Tests und Auswahlverfahren (Stuttgart: Gustav Fischer Verlag)

Houdek, G., Balmforth, N. J., Christensen-Dalsgaard, J., \& Gough, D. O. 1999, A\&A, 351, 582

Jenkins, J. M., Caldwell, D. A., Chandrasekaran, H., et al. 2010a, ApJ, 713, L120

Jenkins, J. M., Caldwell, D. A., Chandrasekaran, H., et al. 2010b, ApJ, 713, L87

Kallinger, T., \& Matthews, J. M. 2010, ApJ, 711, L35

Kaye, A. B., Handler, G., Krisciunas, K., Poretti, E., \& Zerbi, F. M. 1999a, PASP, 111,840

Kaye, A. B., Henry, G. W., Fekel, F. C., \& Hall, D. S. 1999b, MNRAS, 308, 1081

Keiner, J., Kunis, S., \& Potts, D. 2009, in Using NFFT 3 - a software library for various nonequispaced fast Fourier transforms ACM Trans. Math. Software, 36,4

Kharchenko, N. V., \& Roeser, S. 2009, VizieR On-line Data Catalog: I/280B

King, H., Matthews, J. M., Rowe, J. F., et al. 2006, CoAst, 148, 28

Koch, D. J., Borucki, W. J., Basri, G., et al. 2010, ApJ, 713, L79

Kolenberg, K., Szabó, R., Kurtz, D., et al. 2010, ApJ, 713, L198

Kurucz, R. L. 1993, Kurucz CD-ROM 13 (Cambridge, USA: Smithonian Astrophysical Observatory)

Lafrasse, S., Mella, G., Bonneau, D., et al. 2010, in Optical and Infrared Interferometry II., ed. W. C. Danchi, F. Delplancke, \& J. K. Rajagopal, Proc. SPIE, 7734, 140

Lampens, P., \& Boffin, H. M. J. 2000, in Delta Scuti and Related Stars, ed. M. Breger, \& M. H. Montgomery, ASP Conf. Ser., 210, 309

Landstreet, J. D., Kupka, F., Ford, H. A., et al. 2009, A\&A, 503, 973

Latham, D. W., Brown, T. M., Monet, D. G., et al. 2005, BAAS, 37, 1340

Lehmann, H., Tkachenko, A., Semaan, T., et al. 2011, A\&A, 526, A124

Lenz, P., \& Breger, M. 2005, CoAst, 146, 53

Lignières, F., Rieutord, M., \& Reese, D. 2006, A\&A, 455, 607

Lindoff, U. 1972, A\&A, 16, 315

Löffler, W. 2000, in The Impact of Large-Scale Surveys on Pulsating Star Research, ed. L. Szabados, \& D. Kurtz, ASP Conf. Ser., 203, 447

Macrae, D. A. 1952, ApJ, 116, 592

Magalashvili, N. L., \& Kumishvili, J. I. 1976, IAU Inform. Bull. Var. Stars, 1167, 1

Malkov, O. Y., Oblak, E., Snegireva, E. A., \& Torra, J. 2006, A\&A, 446, 785

Martín, S., Bossi, M., \& Zerbi, F. M. 2003, A\&A, 401, 1077

Masana, E., Jordi, C., \& Ribas, I. 2006, A\&A, 450, 735

Mason, B. D., Wycoff, G. L., Hartkopf, W. I., Douglass, G. G., \& Worley, C. E. 2001, AJ, 122, 3466

Mathias, P., Le Contel, J.-M., Chapellier, E., et al. 2004, A\&A, 417, 189

Molenda-Żakowicz, J., Frasca, A., \& Latham, D. W. 2008, AcA, 58, 419

Molenda-Żakowicz, J., Latham, D. W., Catanzaro, G., Frasca, A., \& Quinn, S. N. 2011, MNRAS, 412, 1210

Monnier, J. D., Townsend, R. H. D., Che, X., et al. 2010, ApJ, 725, 1192

Moon, T. T. 1985, Comm. from the Univ. of London Obs., 78

Moore, J. H. \& Paddock, G. F. 1950, ApJ, 112, 48

Moya, A., \& Rodríguez-López, C. 2010, ApJ, 710, L7

Moya, A., Suárez, J. C., Amado, P. J., et al. 2005, A\&A, 432, 289

Niemczura, E., Morel, T., \& Aerts, C. 2009, A\&A, 506, 213

Nordström, B., Mayor, M., Andersen, J., et al. 2004, A\&A, 418, 989

Otero, S. 2007, Open European Journal on Variable stars, 72, 10

Østensen, R. H., Silvotti, R., Charpinet, S., et al. 2010, MNRAS, 409, 1470

Pereira, T. M. D., Suárez, J. C., Lopes, I., et al. 2007, A\&A, 464, 659

Perryman, M. A. C., Lindegren, L., Kovalevsky, J., et al. 1997, A\&A, 323, L49 Pigulski, A., Pojmański, G., Pilecki, B., \& Szczygieł, D. M. 2009, AcA, 59, 33

Poretti, E., Koen, C., Martinez, P., et al. 1997, MNRAS, 292, 621

Poretti, E., Garido, R., Amado, P. J., et al. 2003, A\&A, 406, 203
Poretti, E., Michel, E., Garrido, R., et al. 2009, A\&A, 506, 85

Press, W. H., Teukolsky, S. A., Vettering, W. T., \& Flannery, B. P. 1992, Numerical Recipes, 2nd edn. (New York: Cambridge University Press)

Prša, A., Batalha, N. M., Slawson, R. W., et al. 2011, AJ, 141, 83

Reegen, P. 2007, A\&A, 467, 1353

Reegen, P. 2011, CoAst, in press [arXiv: 1006. 5081]

Rodríguez, E., \& Breger, M. 2001, A\&A, 366, 178

Rodríguez, E., López-González, M. J., \& López de Coca, P. 2000, A\&AS, 144, 469

Rowe, J. F., Matthews, J. M., Cameron, C., et al. 2006, CoAst, 148, 34

Samadi, R., Goupil, M.-J., \& Houdek, G. 2002, A\&A, 395, 563

Sato, K., \& Kuji, S. 1990, A\&AS, 85, 1069

Scargle, J. D. 1982, ApJ, 263, 835

Scuflaire, R., Théado, S., Montalbán, J., et al. 2008, A\&AS, 316, 83

Shulyak, D., Tsymbal, V., Ryabchikova, T., Stütz, Ch., \& Weiss, W. W. 2004, A\&A, 428, 993

Skiff, B. A. 2007, Vizier On-line Data Catalog, 1, 2023

Slawson, R. W., Prša, A., Welsh, W. F., et al. 2011, AJ, in press [arXiv: 1103. 1659]

Smalley, B., \& Kupka, F. 1997, A\&A, 328, 349

Soubiran, C., Le Campion, J.-F., Cayrel de Strobel, G., \& Caillo, A. 2010, A\&A, 515, A111

Stephenson, C. B. 1986, ApJ, 301, 927

Strömgren, B. 1966, ARA\&A, 4, 433

Suárez, J. C., Moya, A., Martín-Ruíz, S., et al. 2005, A\&A, 443, 271

Szabó, R., Szabados, L., Ngeow, C.-C., et al. 2011, MNRAS, 413, 2709

Tango, W. J., Davis, J., Ireland, M., et al. 2006, MNRAS, 370, 884

Trampedach, R., \& Stein, R. F. 2011, ApJ, 731, A78

Tsymbal, V. 1996, ASPC, 108, 198

Turcotte, S., Richer, J., Michaud, G., \& Christensen-Dalsgaard, J. 2000, A\&A, 360,603

Unno, W., Osaki, Y., Ando, H., Saio, H., \& Shibahashi, H. 1989, Nonradial oscillations of stars (Tokyo: University of Tokyo Press)

Uytterhoeven, K., Telting, J. H., Aerts, C., \& Willems, B. 2004, A\&A, 427, 593

Uytterhoeven, K., Mathias, P., Poretti, E., et al. 2008, A\&A, 489, 2213

Uytterhoeven, K., Szabo, R., Southworth, J., et al. 2010a, AN, 331, P30 [arXiv: 1003.6089]

Uytterhoeven, K., Briquet, M., Bruntt, H., et al. 2010b, AN, 331, 993

Vyssotsky, A. N. 1958, Publications of the Leander Mc Cormik Observatory of the University of Virginia, Charlottesville, 13, V

Walker, G., Matthews, J., Kuschnig, R., et al. 2003, PASP, 115, 1023

Watson, C. L. 2006, SASS, 25, 47, AAVSO International Variable Star Index VSX, Watson+, 2006-2010

Worley, C. E., \& Douglass, G. G. 1997, A\&AS, 125, 523

Wright, C. O., Egan, M. P., Kraemer, K. E., \& Price, S. D. 2003, AJ, 125, 359

Zechmeister, M., \& Kürster, M. 2009, A\&A, 496, 577

Zerbi, F. M., Rodríguez, E., Garrido, R., et al. 1997, MNRAS, 292, 43

Zerbi, F. M., Rodríguez, E., Garrido, R., et al. 1999, MNRAS, 303, 275

1 Laboratoire AIM, CEA/DSM-CNRS-Université Paris Diderot; CEA, IRFU, SAp, Centre de Saclay, 91191, Gif-sur-Yvette, France

2 Kiepenheuer-Institut für Sonnenphysik, Schöneckstraße 6, 79104 Freiburg im Breisgau, Germany

3 Instituto de Astrofísica de Canarias, 38200 La Laguna, Tenerife, Spain; Departamento de Astrofísica, Universidad de La Laguna, 38205 La Laguna, Tenerife, Spain

e-mail: katrien@iac.es

4 Laboratorio de Astrofísica Estelar y Exoplanetas, LAEX-CAB (INTA-CSIC), PO BOX 78, 28691 Villanueva de la Cañada, Madrid, Spain

5 Centro de Astrofísica, Faculdade de Ciências, Universidade do Porto, Rua das Estrelas, 4150-762 Porto, Portugal

6 Los Alamos National Laboratory, XTD-2, Los Alamos, NM 875452345, USA

7 Instituto de Astrofísica de Andalucía (CSIC), Apartado 3004, 18080 Granada, Spain

8 LESIA, Observatoire de Paris, CNRS, UPMC, Université ParisDiderot, 92195 Meudon, France

9 Valentian International University, Prolongación C/ José Pradas Gallen, s/n 12006 Castellón de la Plana, Spain

10 Astrophysics Group, Keele University, Staffordshire, ST5 5BG, UK

11 Institut für Astronomie, Türkenschanzstraße 17, 1180 Wien, Austria

12 Nicolaus Copernicus Astronomical Center, Bartycka 18, 00-716 Warsaw, Poland 
K. Uytterhoeven et al.: The Kepler characterization of the variability among A- and F-type stars. I.

13 South African Astronomical Observatory, PO Box 9, Observatory 7935, South Africa

14 Instytut Astronomiczny, Uniwersytet Wrocławski, Kopernika 11, 51-622 Wrocław, Poland

15 Observatorio Astronómico Nacional, Instituto de Astronomía, UNAM, Ensenada B.C., Apdo. Postal 877, Mexico

16 CISAS, Padova University, via Venezia 15, 35131 Padova, Italy

17 INAF - Astronomical Observatory of Padova, Vicolo Osservatorio 5, 35122 Padova, Italy

18 UMR 6525 H. Fizeau, UNS, CNRS, OCA, Campus Valrose, 06108 Nice Cedex 2, France

19 Instituut voor Sterrenkunde, K.U.Leuven, Celestijnenlaan 200D, 3001 Leuven, Belgium

20 Konkoly Observatory of the Hungarian Academy of Sciences, 1525 Budapest PO Box 67, Hungary

21 INAF - Osservatorio Astronomico di Capodimonte, via Moiariello 16, 80131 Napoli, Italy

${ }^{22}$ Lab. d'Astrophysique de Toulouse-Tarbes, Université de Toulouse, CNRS, 57 avenue d'Azereix, 65000 Tarbes, France

23 Thüringer Landessternwarte Tautenburg, 07778 Tautenburg, Germany

24 Department of Astronomy, New Mexico State University, Las Cruces, NM 88001, USA

25 Astronomical Institute "Anton Pannekoek", University of Amsterdam, Science Park 904, 1098 XH Amsterdam, The Netherlands
26 University of Birmingham, School of Physics and Astronomy, Edgbaston, Birmingham B15 2TT, UK

27 Department of Astronomy and Physics, Saint Marys University, Halifax, NS B3H 3C3, Canada

28 Observatoire de Genève, Université de Genève, 51 Ch. des Maillettes, 1290 Sauverny, Switzerland

${ }^{29}$ Los Alamos National Laboratory, XCP-6, MS T-087, Los Alamos, NM 87545-2345, USA

30 Jeremiah Horrocks Institute of Astrophysics, University of Central Lancashire, Preston PR1 2HE, UK

31 Royal Observatory of Belgium, Ringlaan 3, 1180 Brussel, Belgium

32 Department of Physics and Astronomy, University of Aarhus, bygn. 1520, Ny Munkegade, 8000 Aarhus C., Denmark

33 Department of Astronomy, University of Texas, Austin, TX 78712, USA

34 Sydney Institute for Astronomy, School of Physics, A28, The University of Sydney, NSW, 2006, Australia

35 Bay Area Environmental Research Inst./NASA Ames Research Center, Moffett Field, CA 94035, USA

36 SETI Institute/NASA Ames Research Center, Moffett Field, CA 94035, USA 


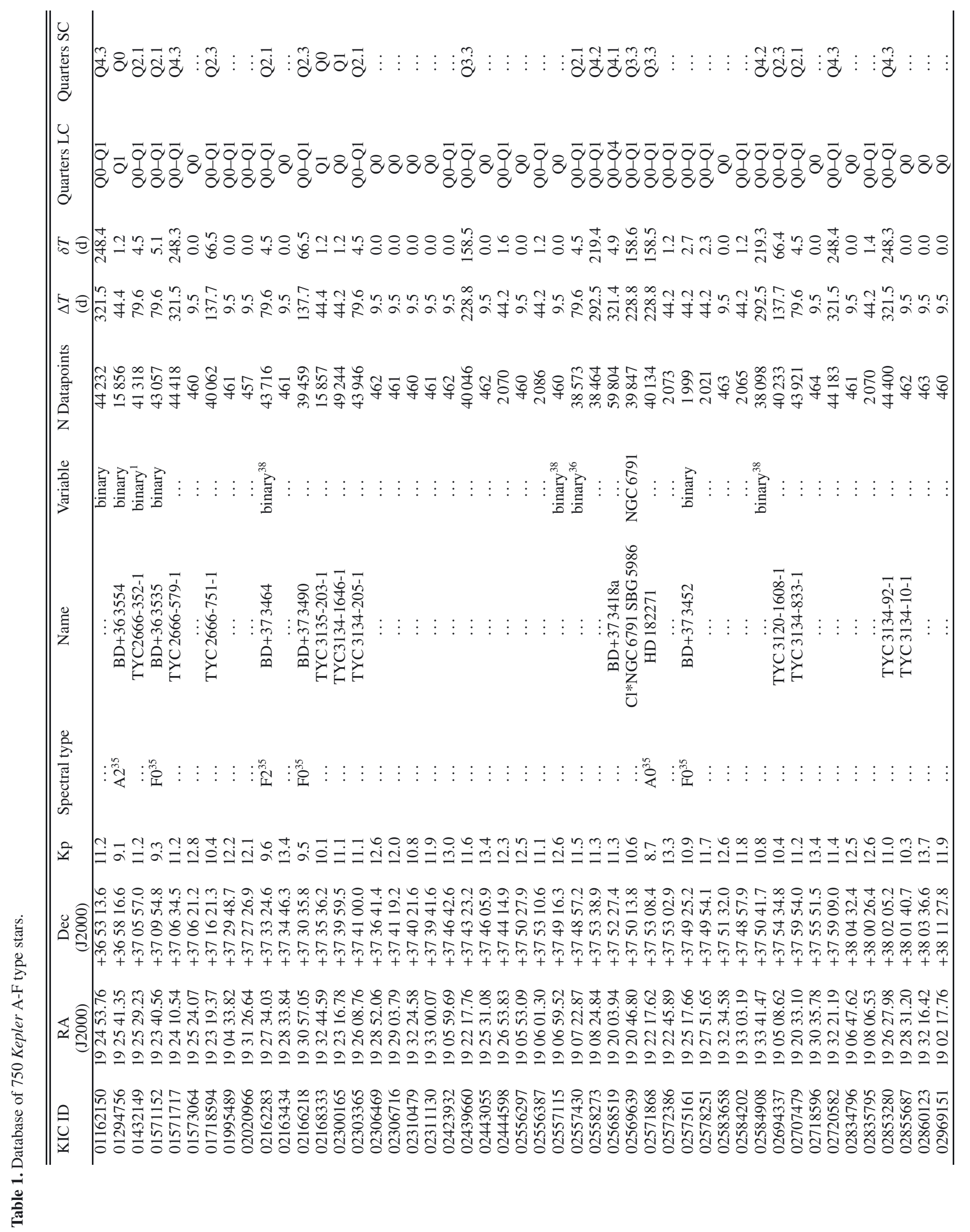


K. Uytterhoeven et al.: The Kepler characterization of the variability among A- and F-type stars. I.

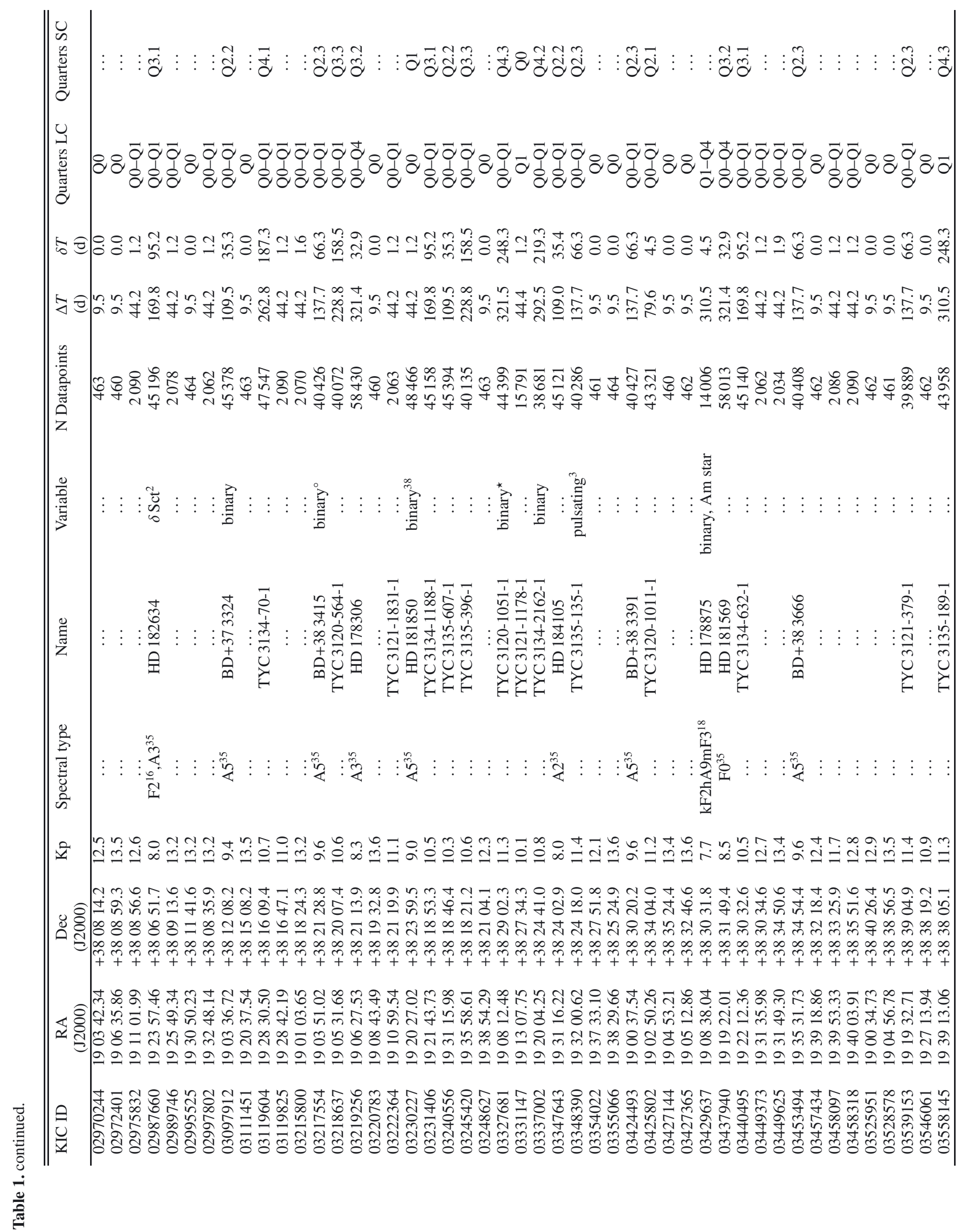




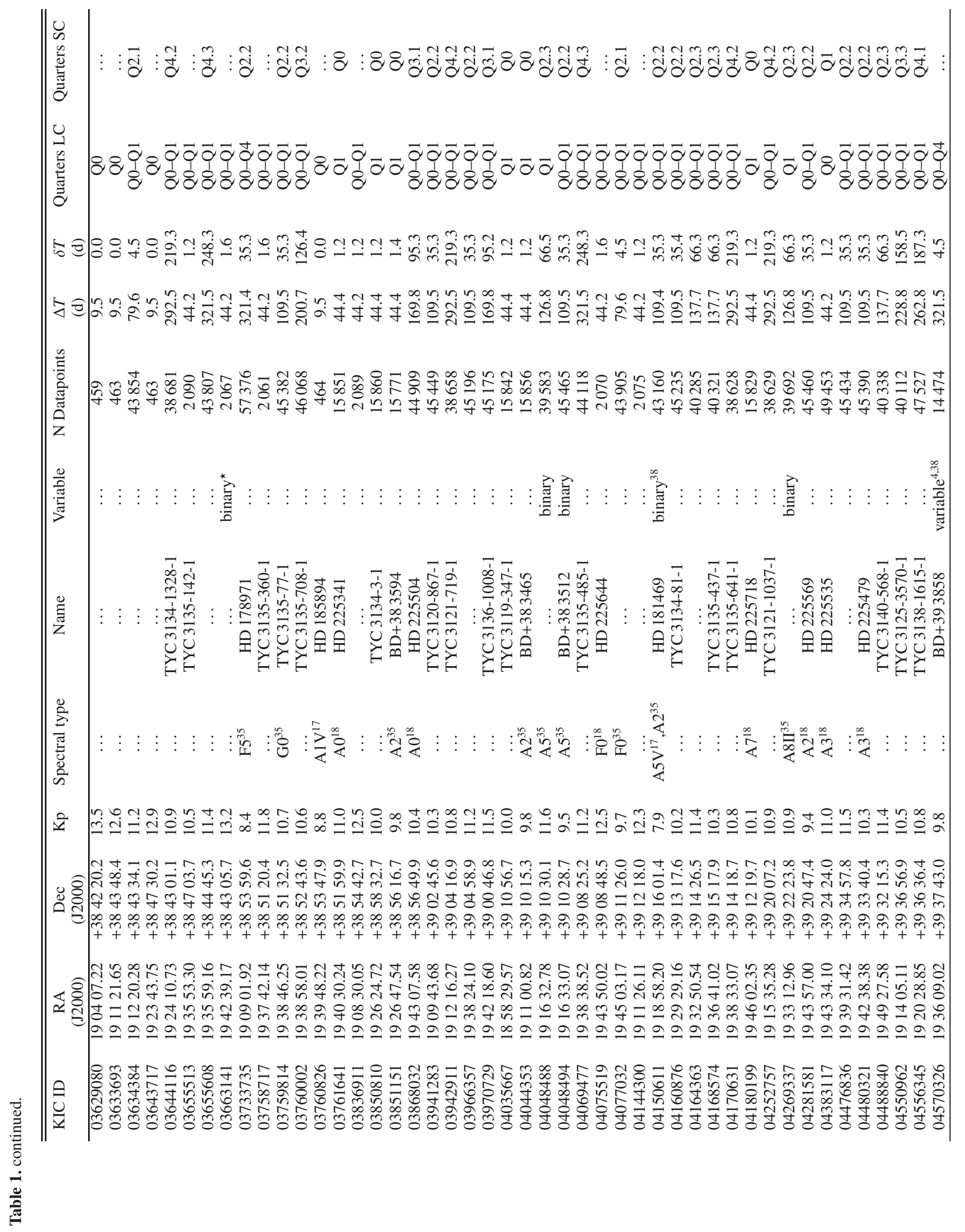


K. Uytterhoeven et al.: The Kepler characterization of the variability among A- and F-type stars. I.

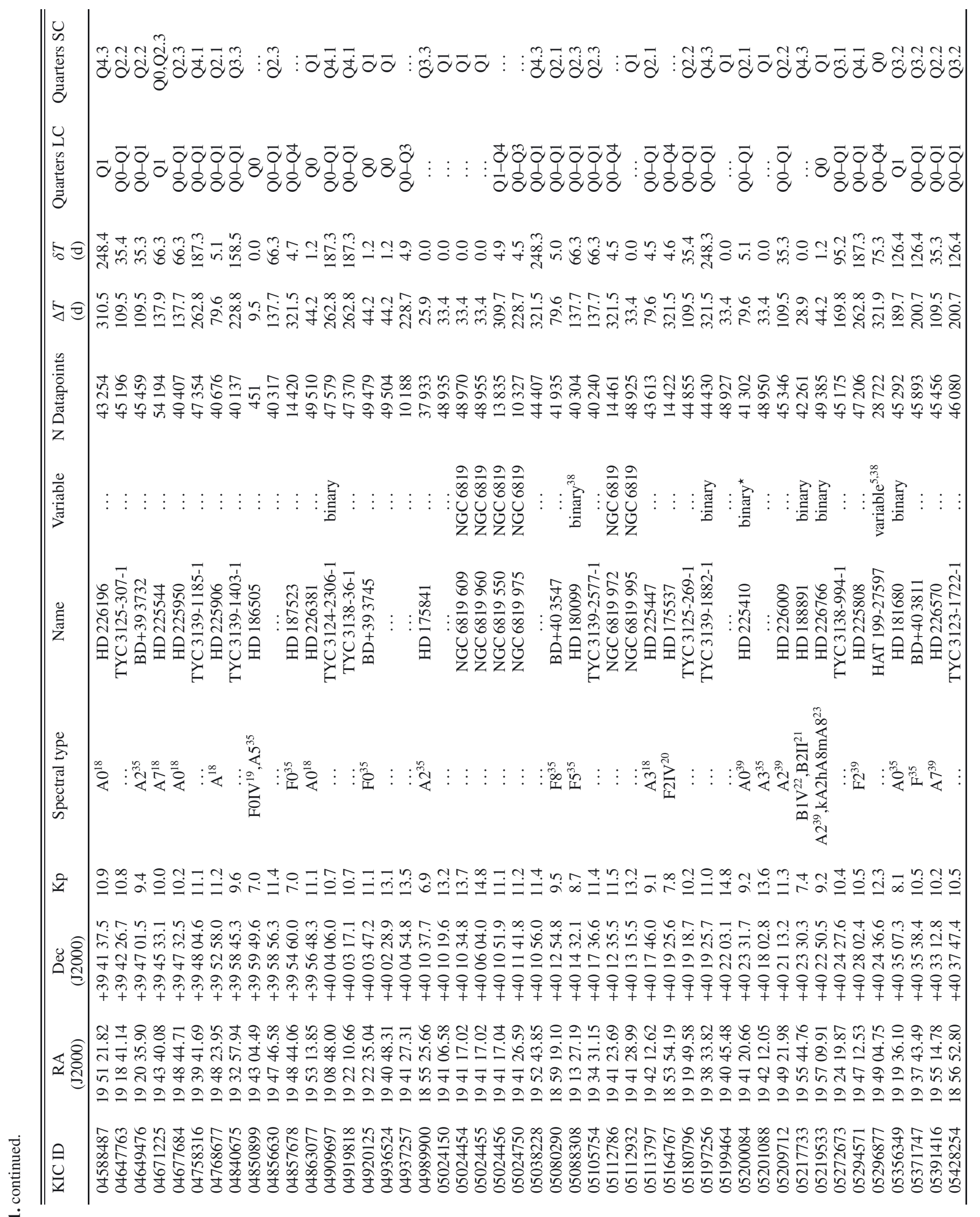




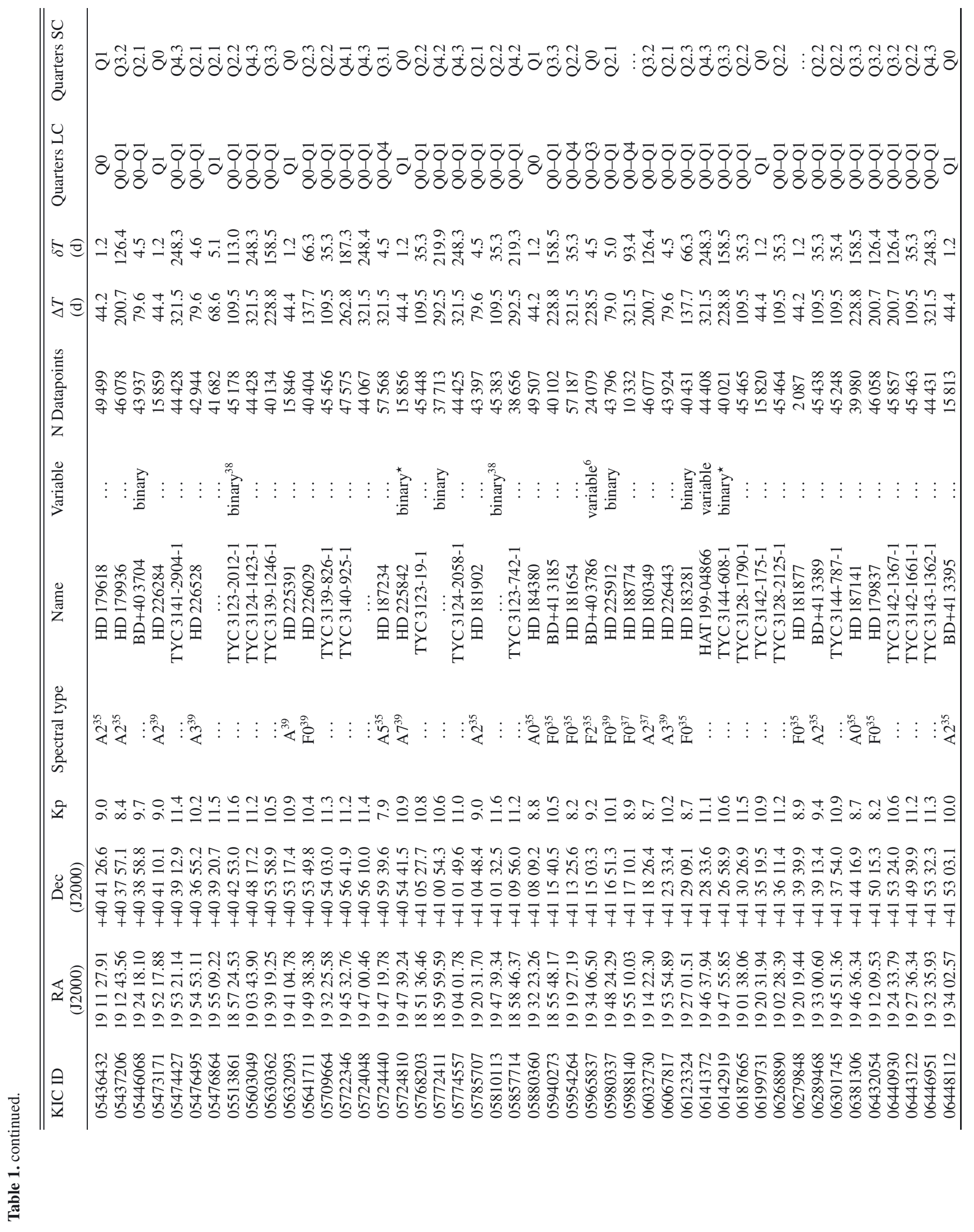


K. Uytterhoeven et al.: The Kepler characterization of the variability among A- and F-type stars. I.

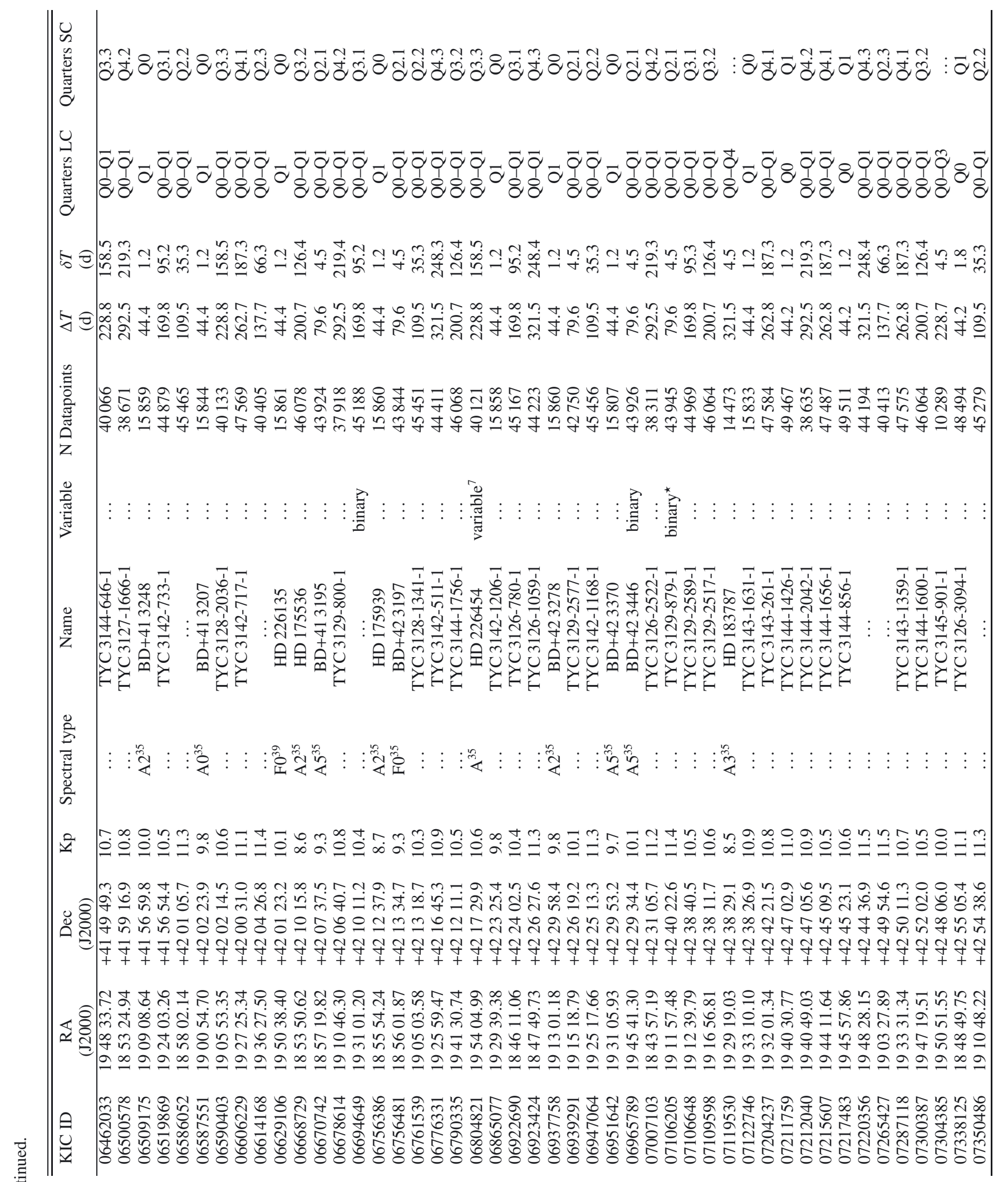




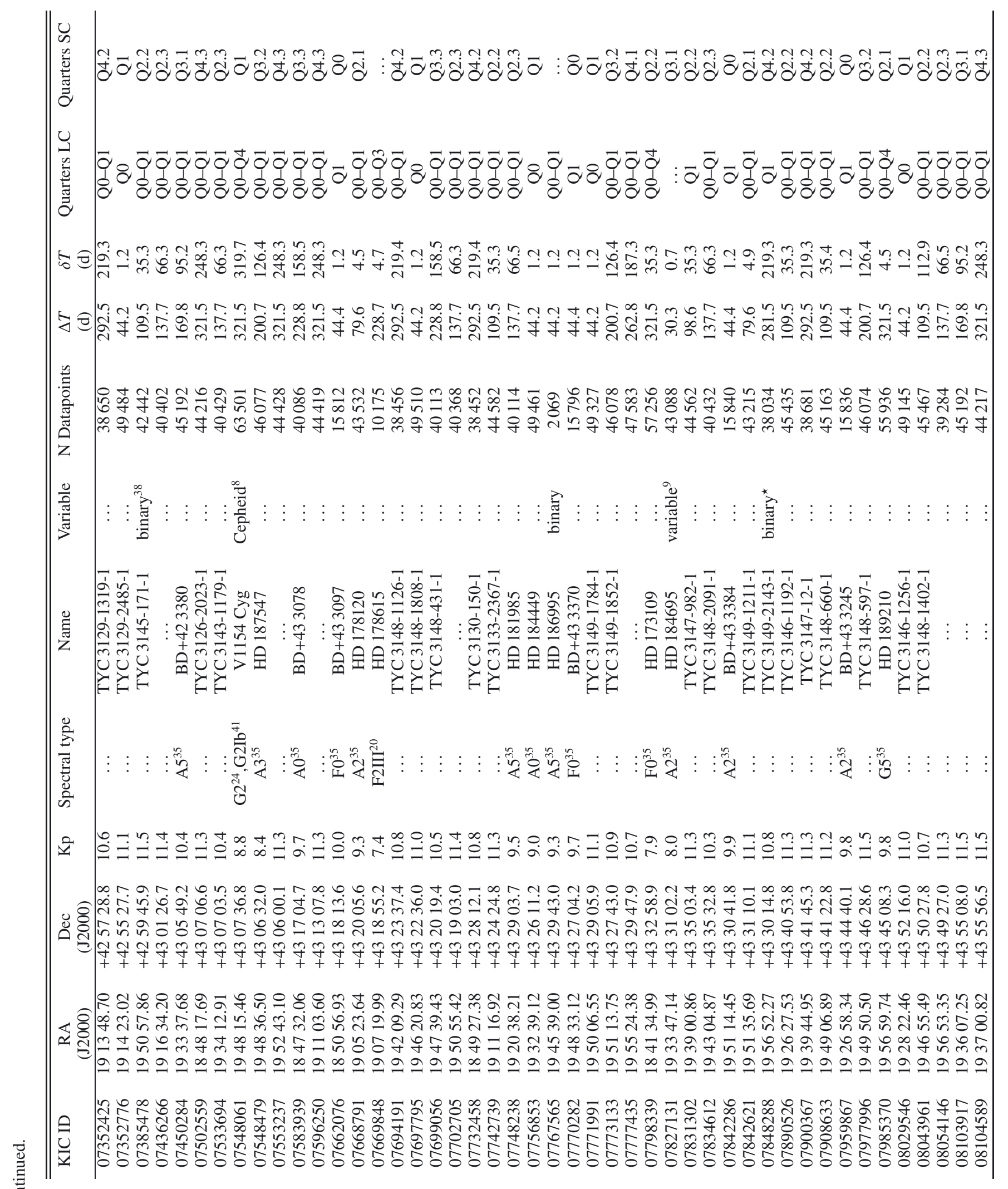


K. Uytterhoeven et al.: The Kepler characterization of the variability among A- and F-type stars. I.

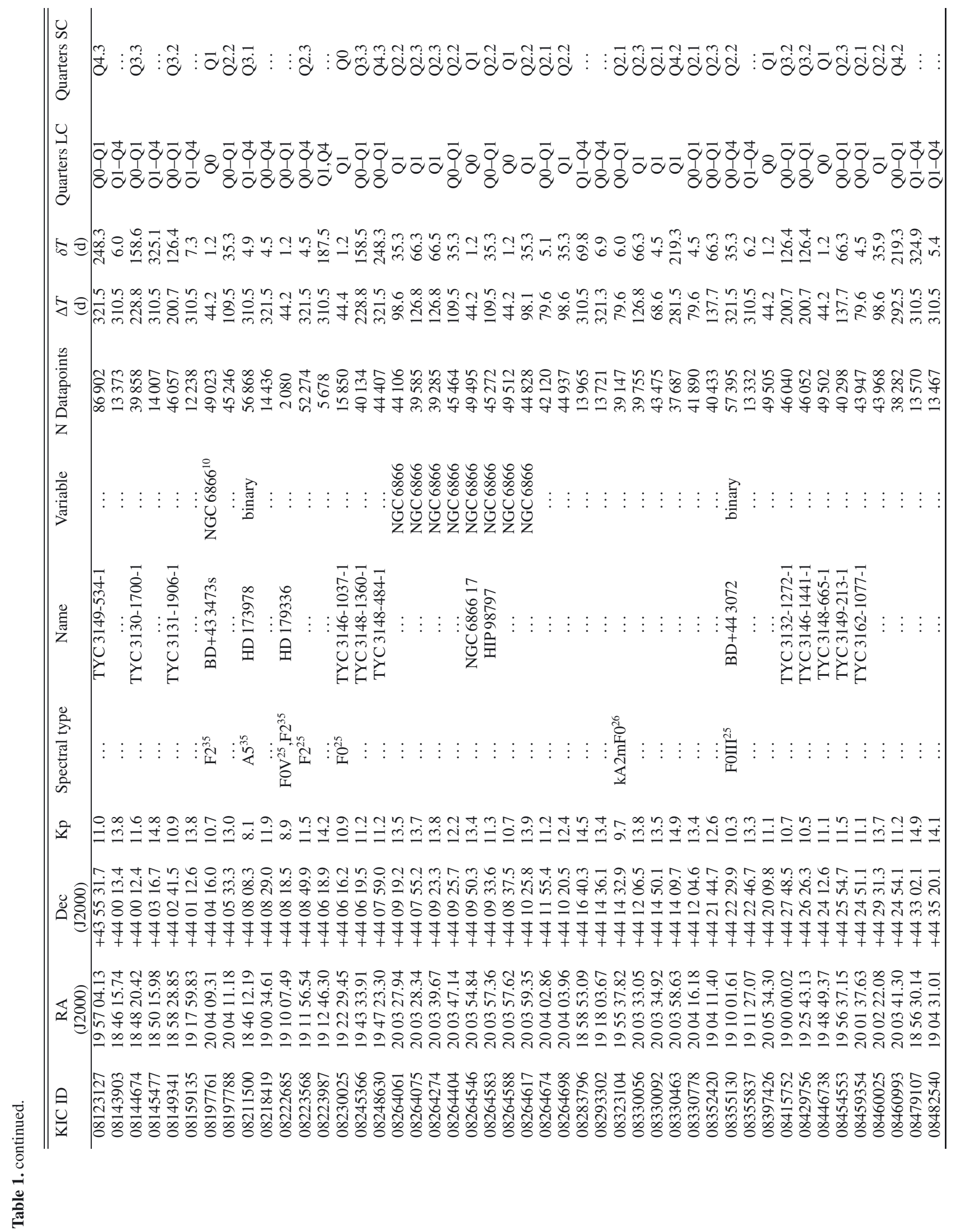




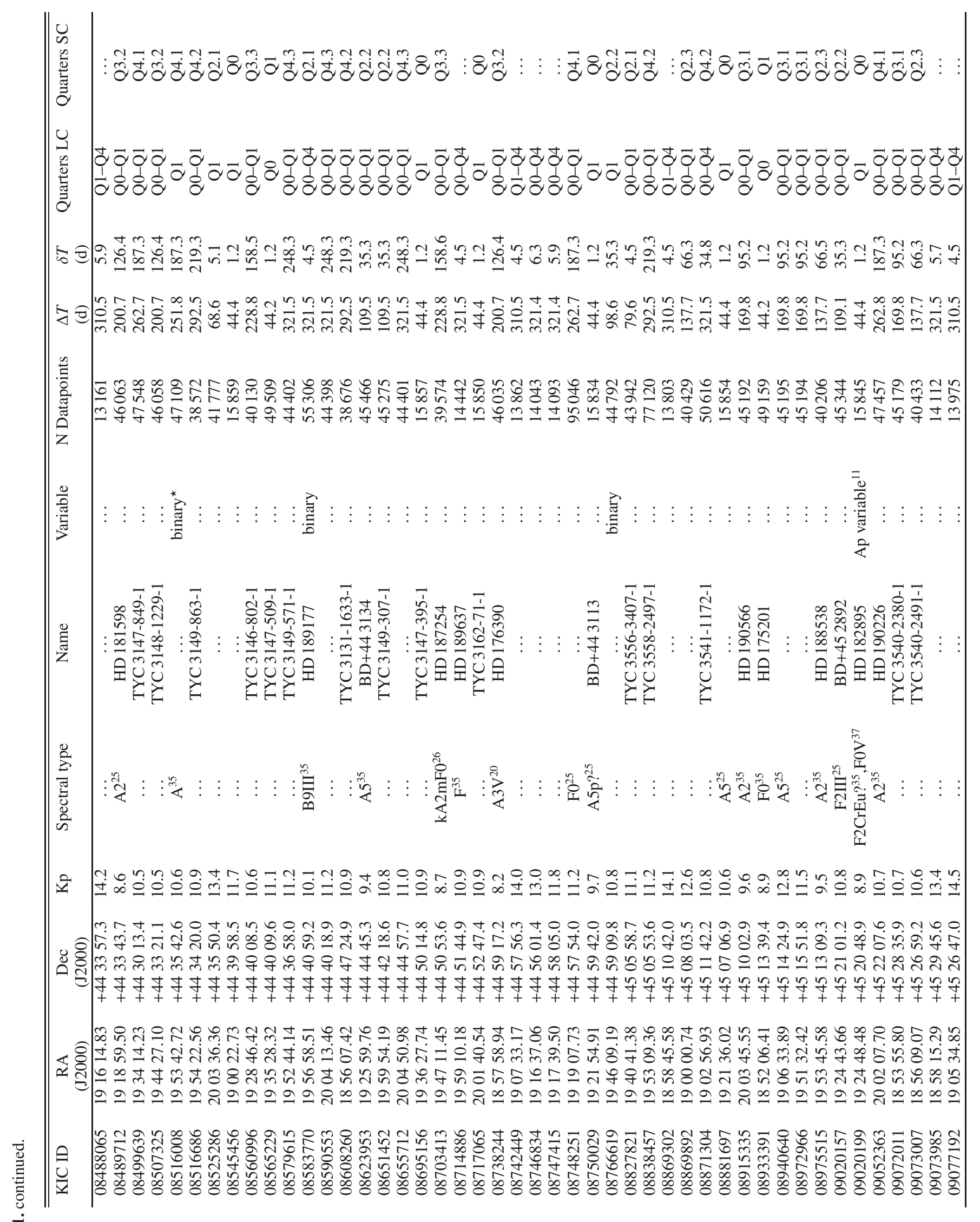


K. Uytterhoeven et al.: The Kepler characterization of the variability among A- and F-type stars. I.

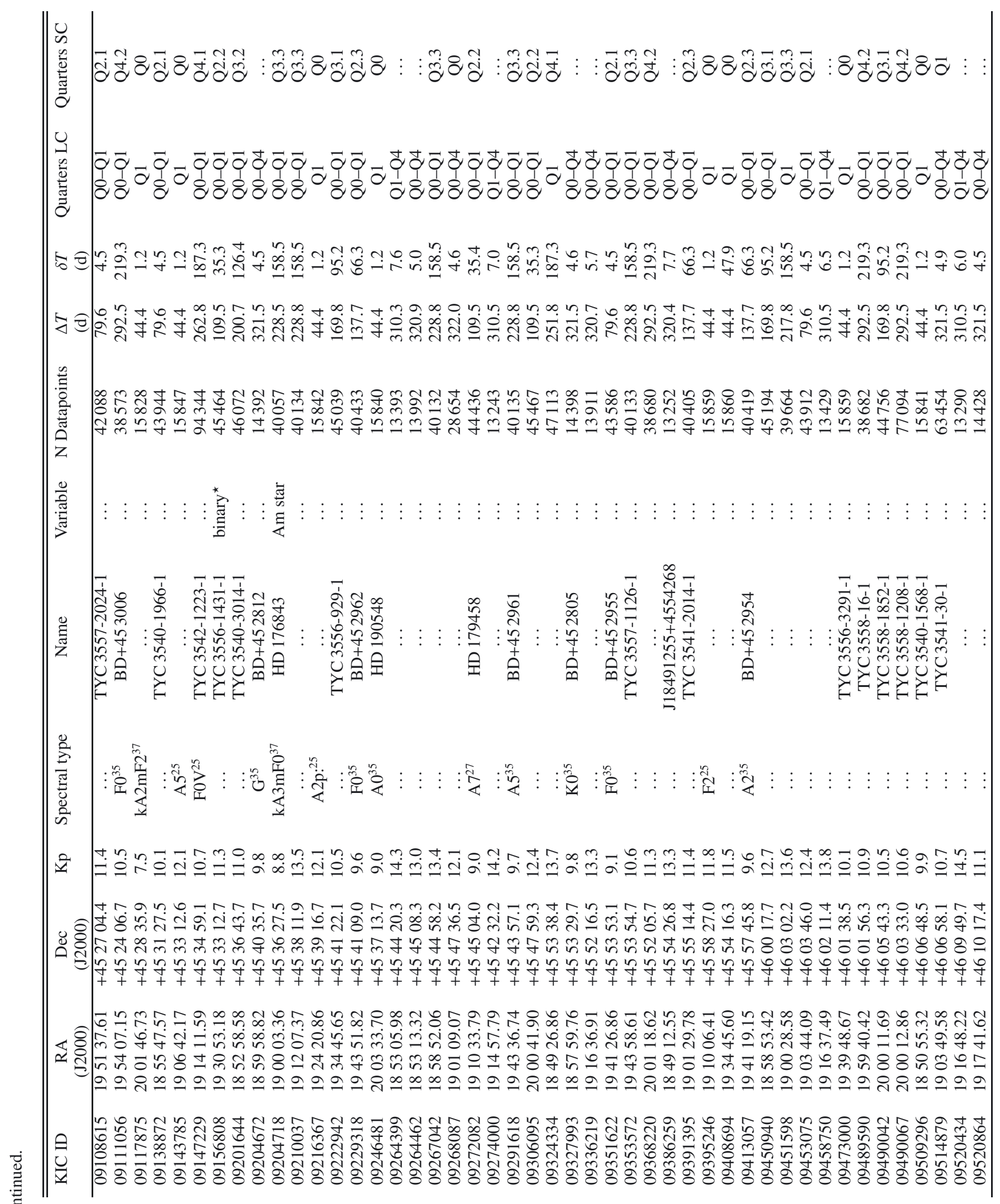




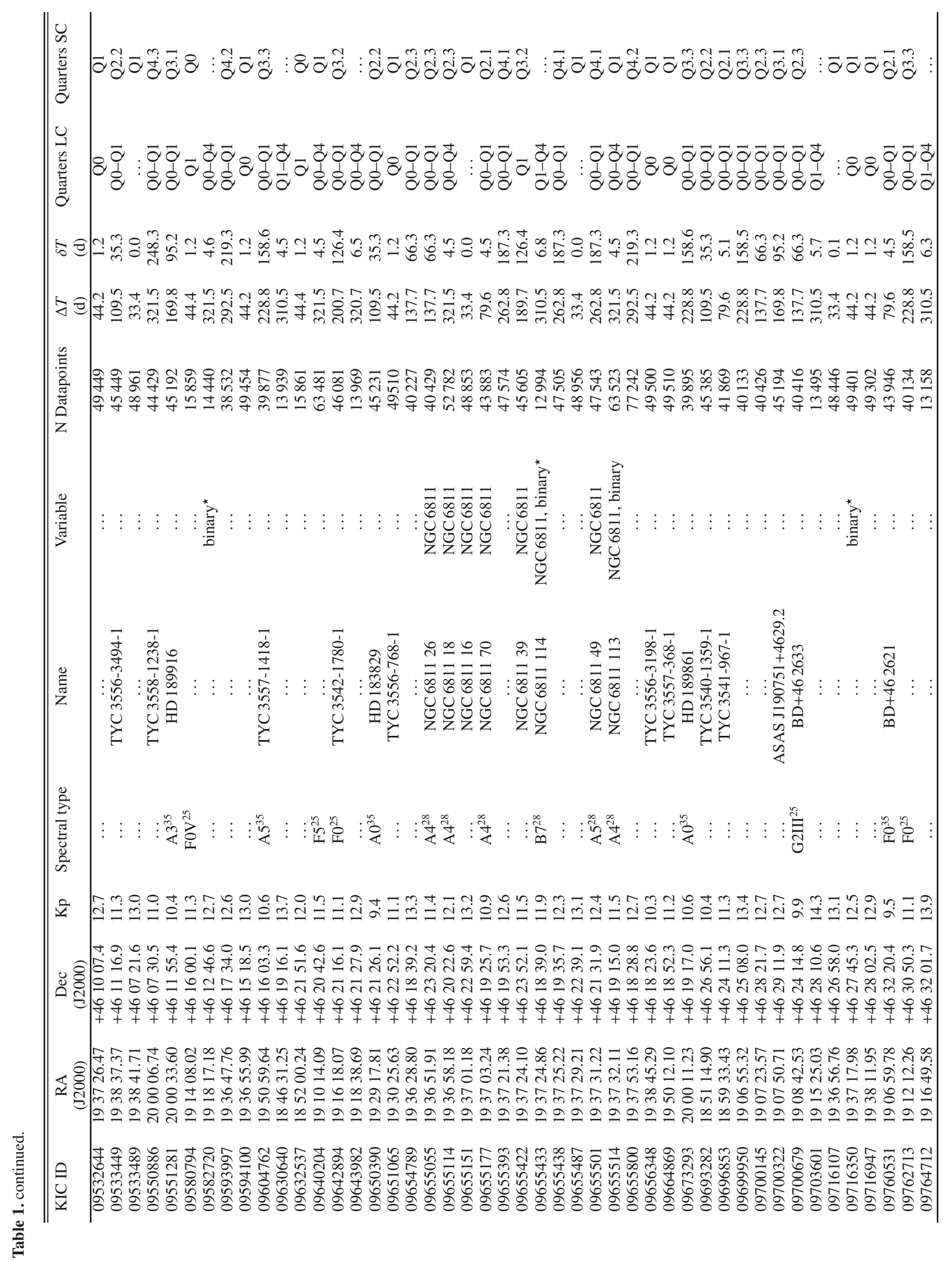


K. Uytterhoeven et al.: The Kepler characterization of the variability among A- and F-type stars. I.

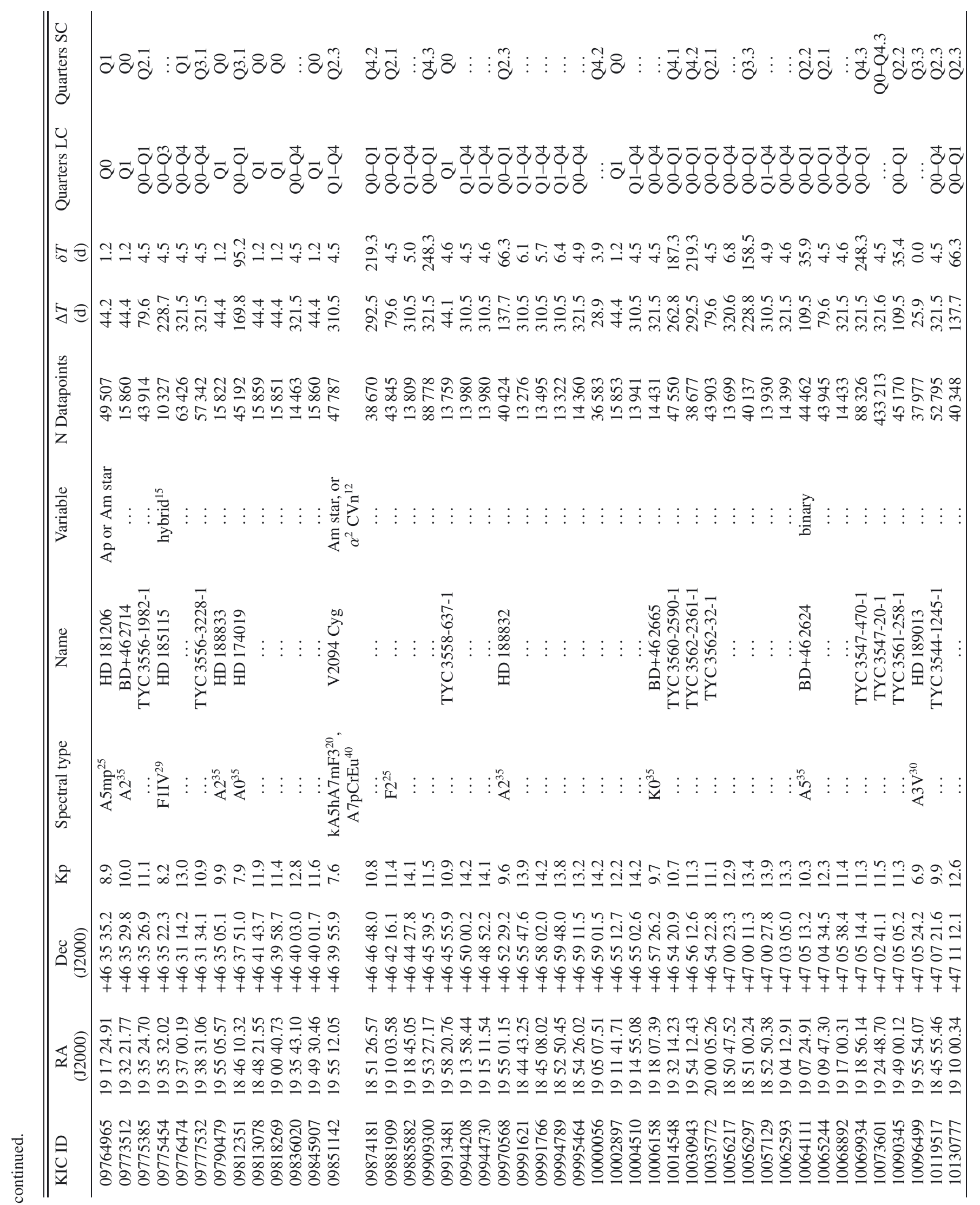




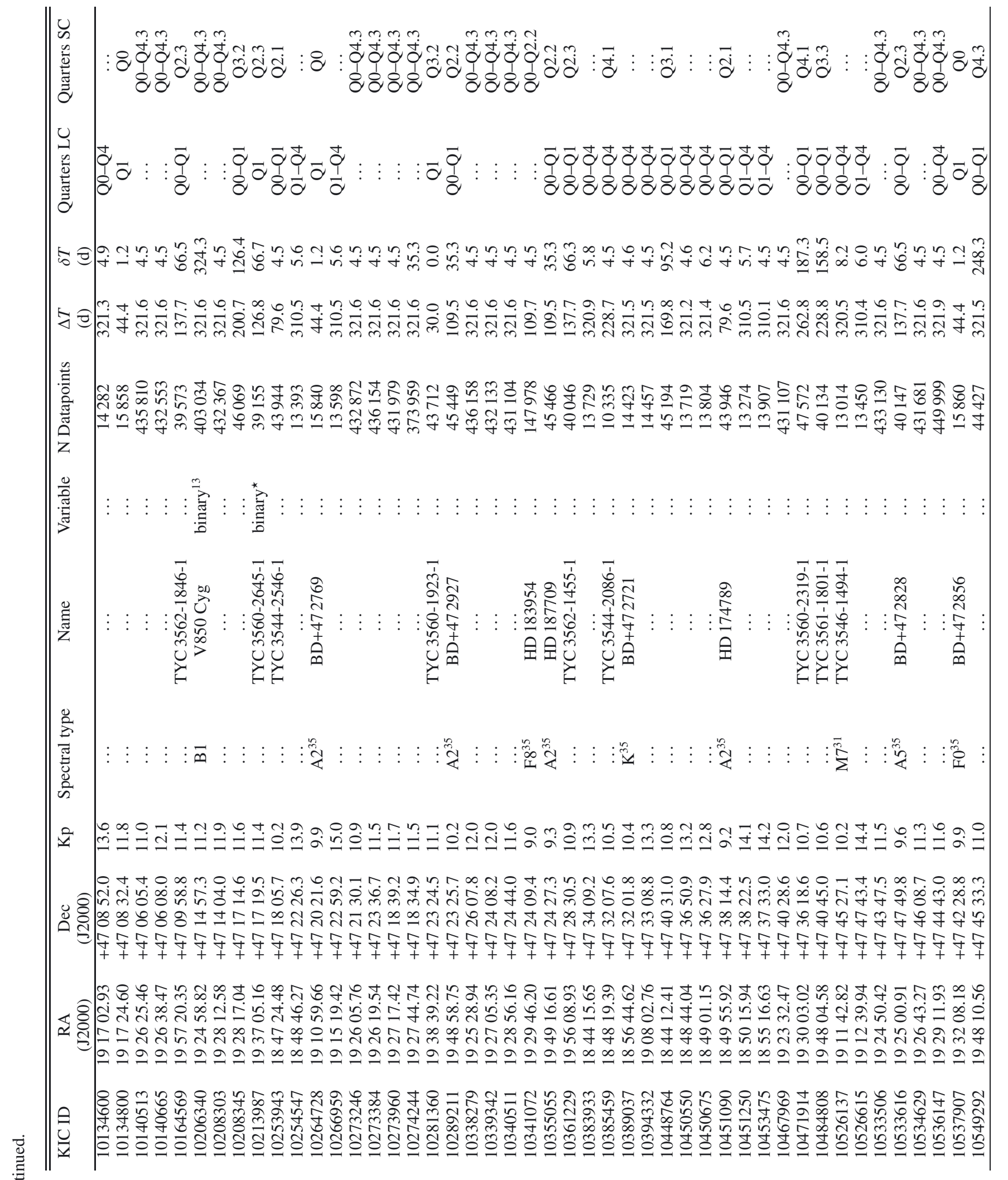




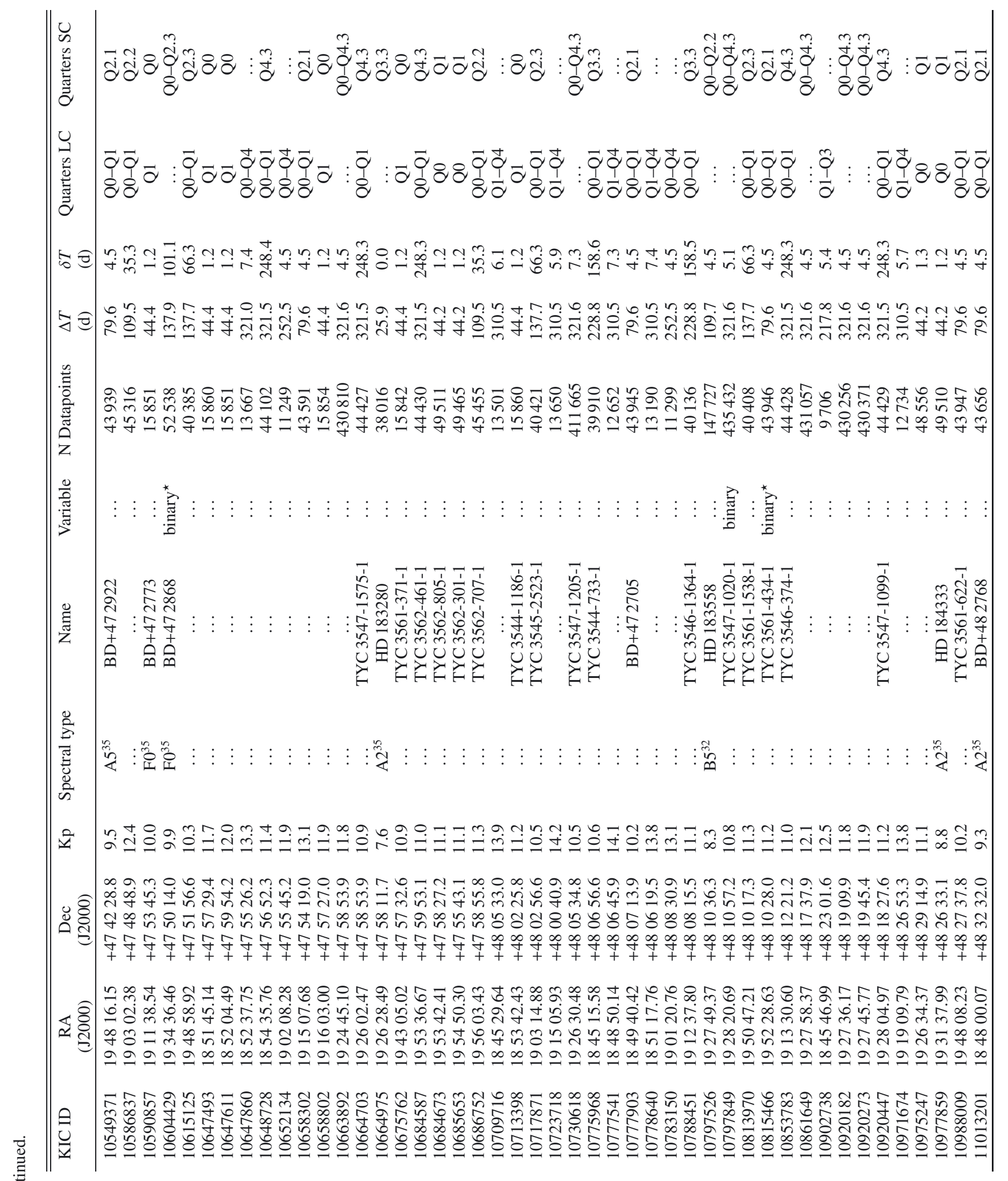




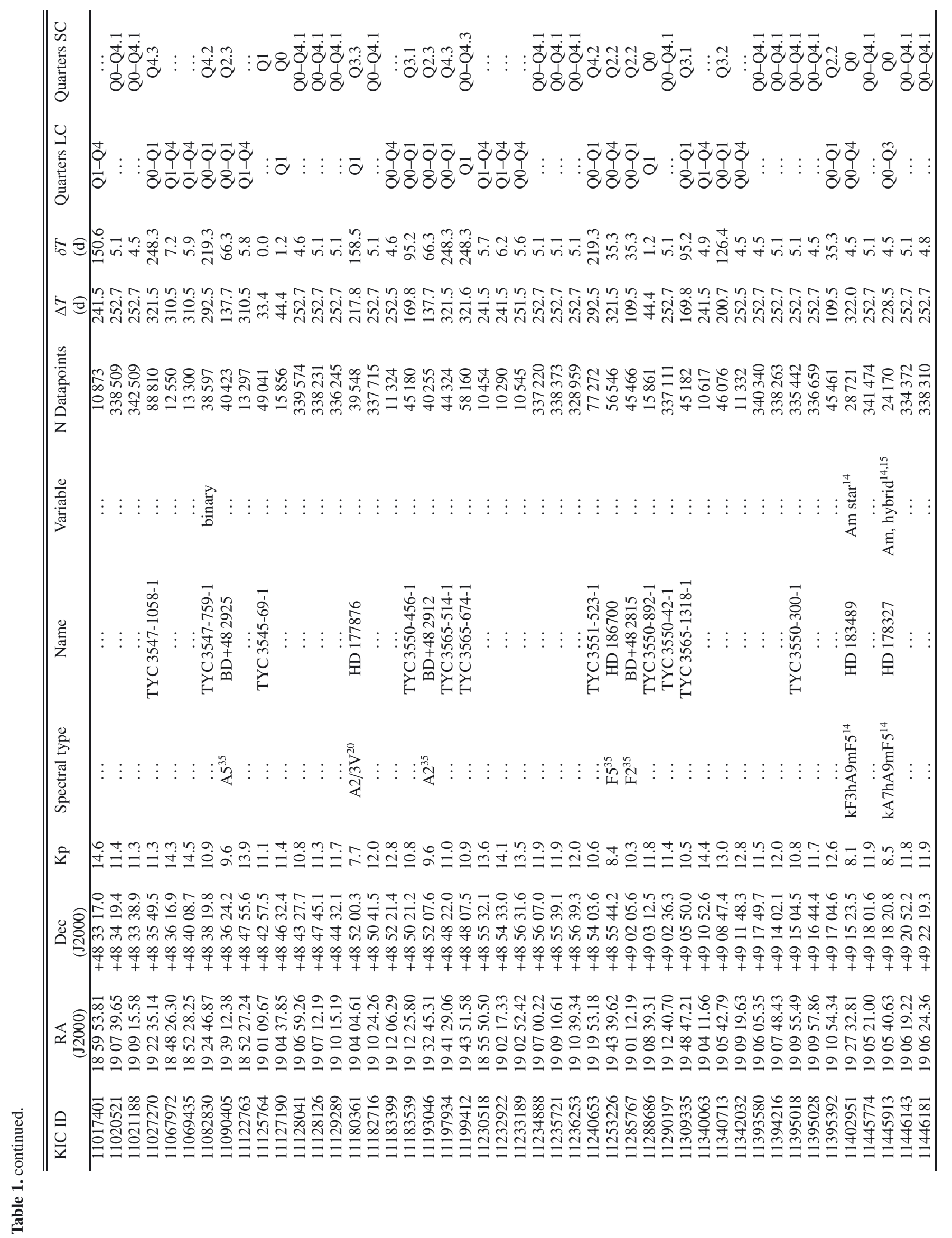


K. Uytterhoeven et al.: The Kepler characterization of the variability among A- and F-type stars. I.

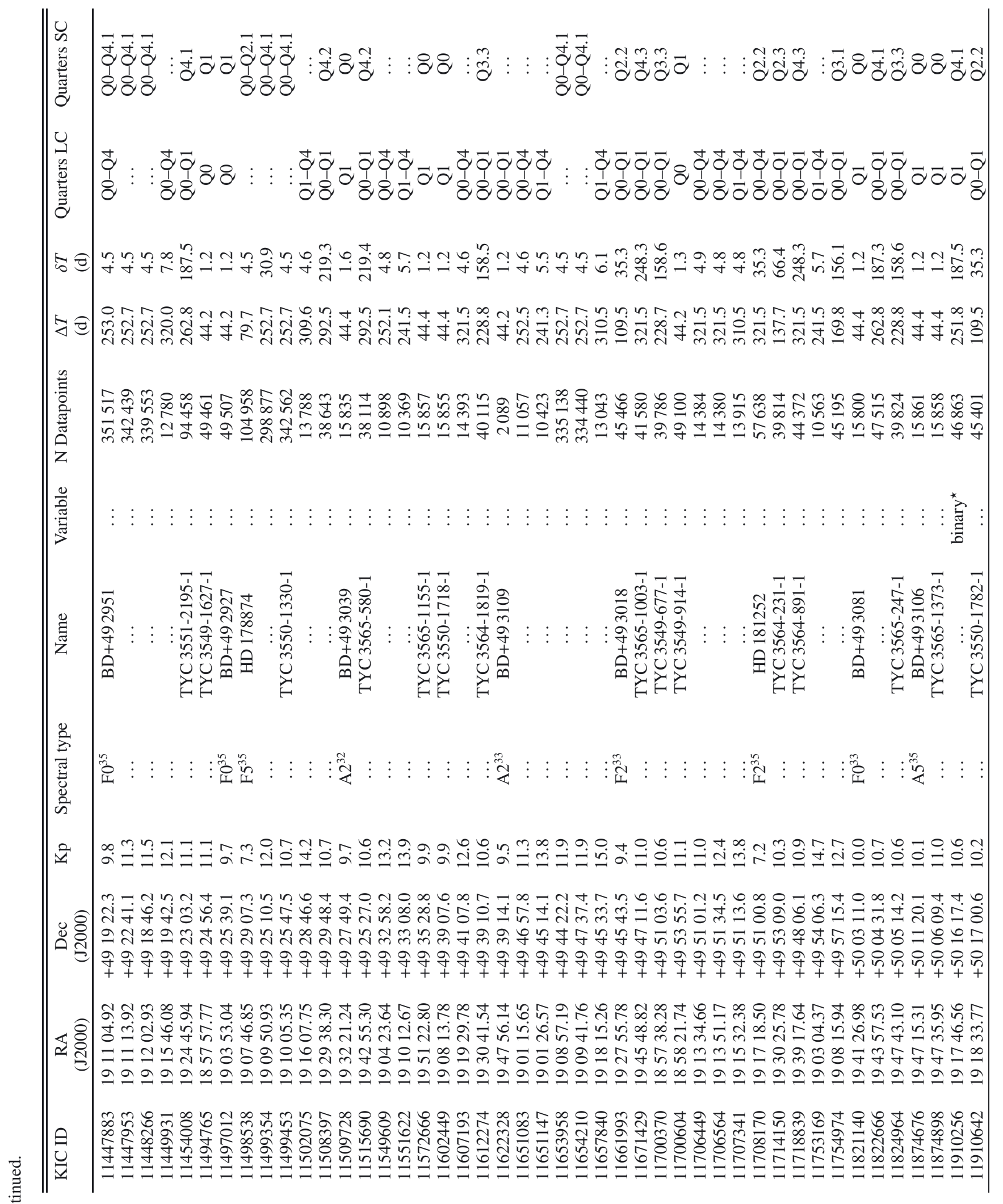




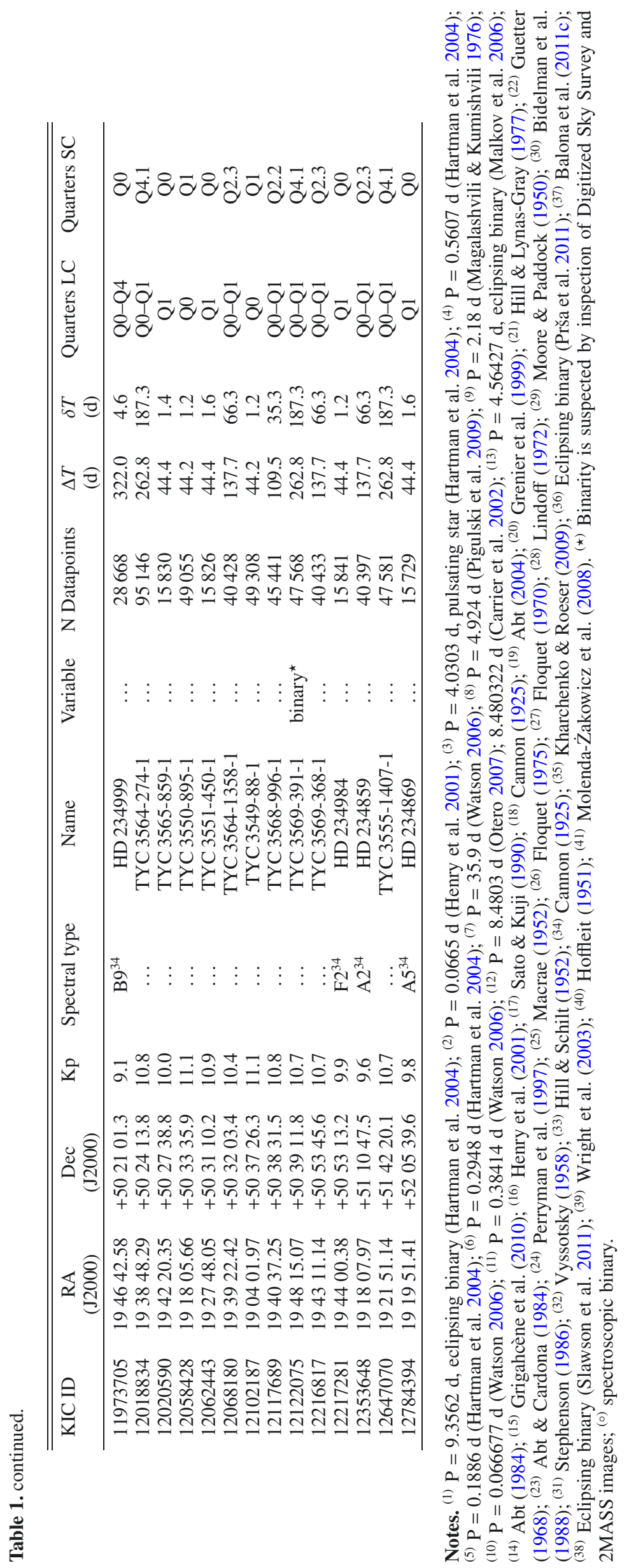


K. Uytterhoeven et al.: The Kepler characterization of the variability among A- and F-type stars. I.

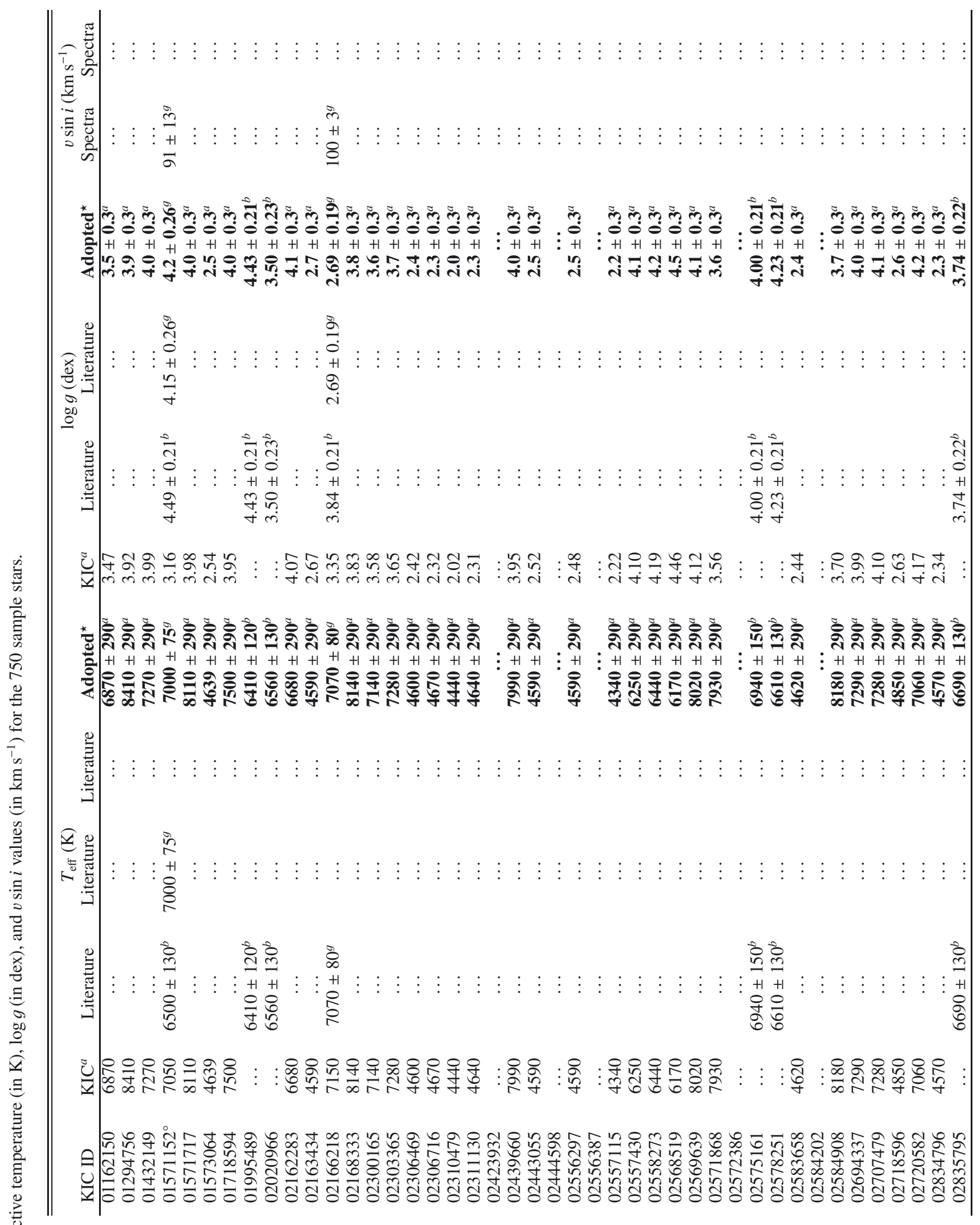




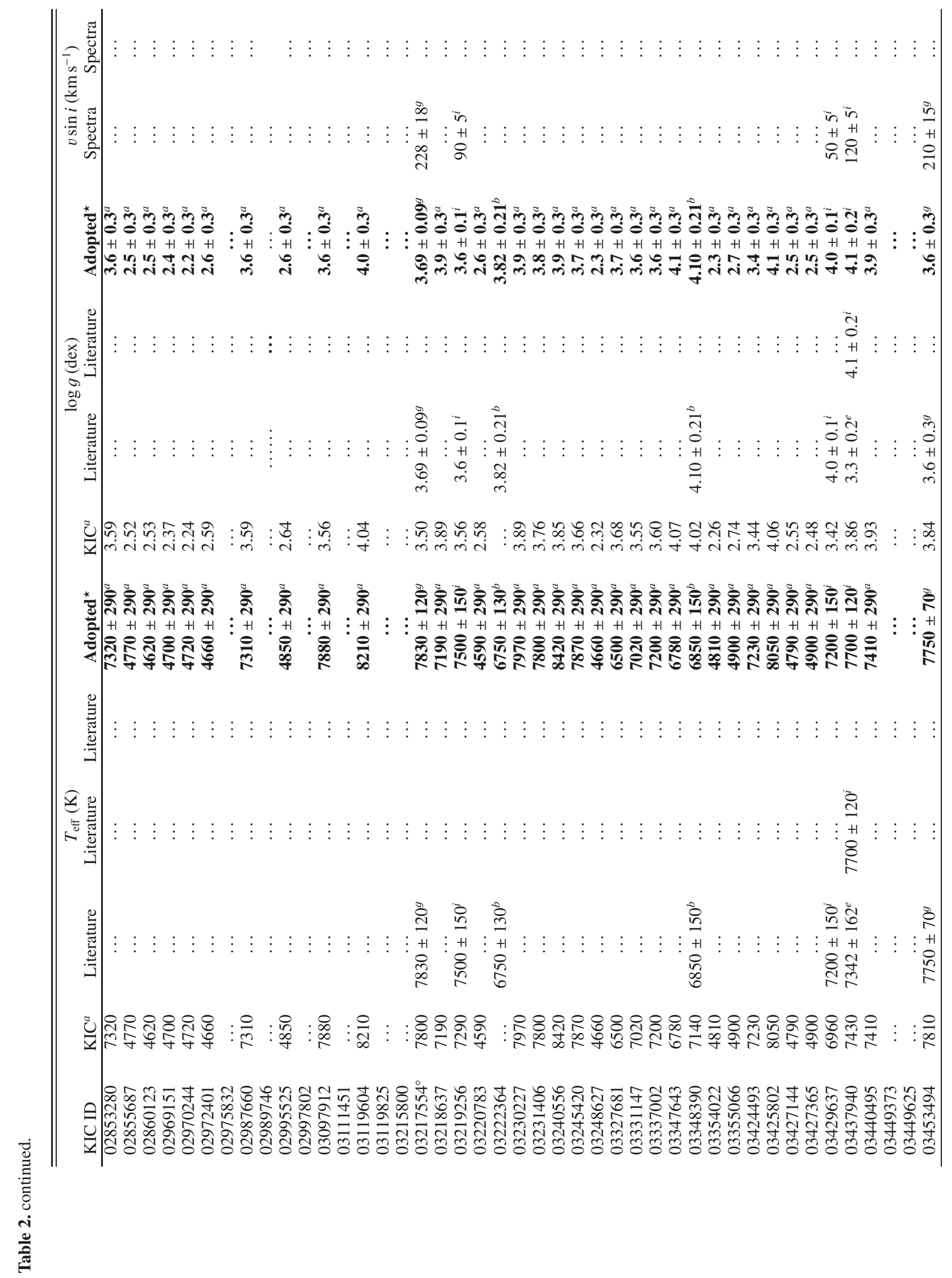


K. Uytterhoeven et al.: The Kepler characterization of the variability among A- and F-type stars. I.

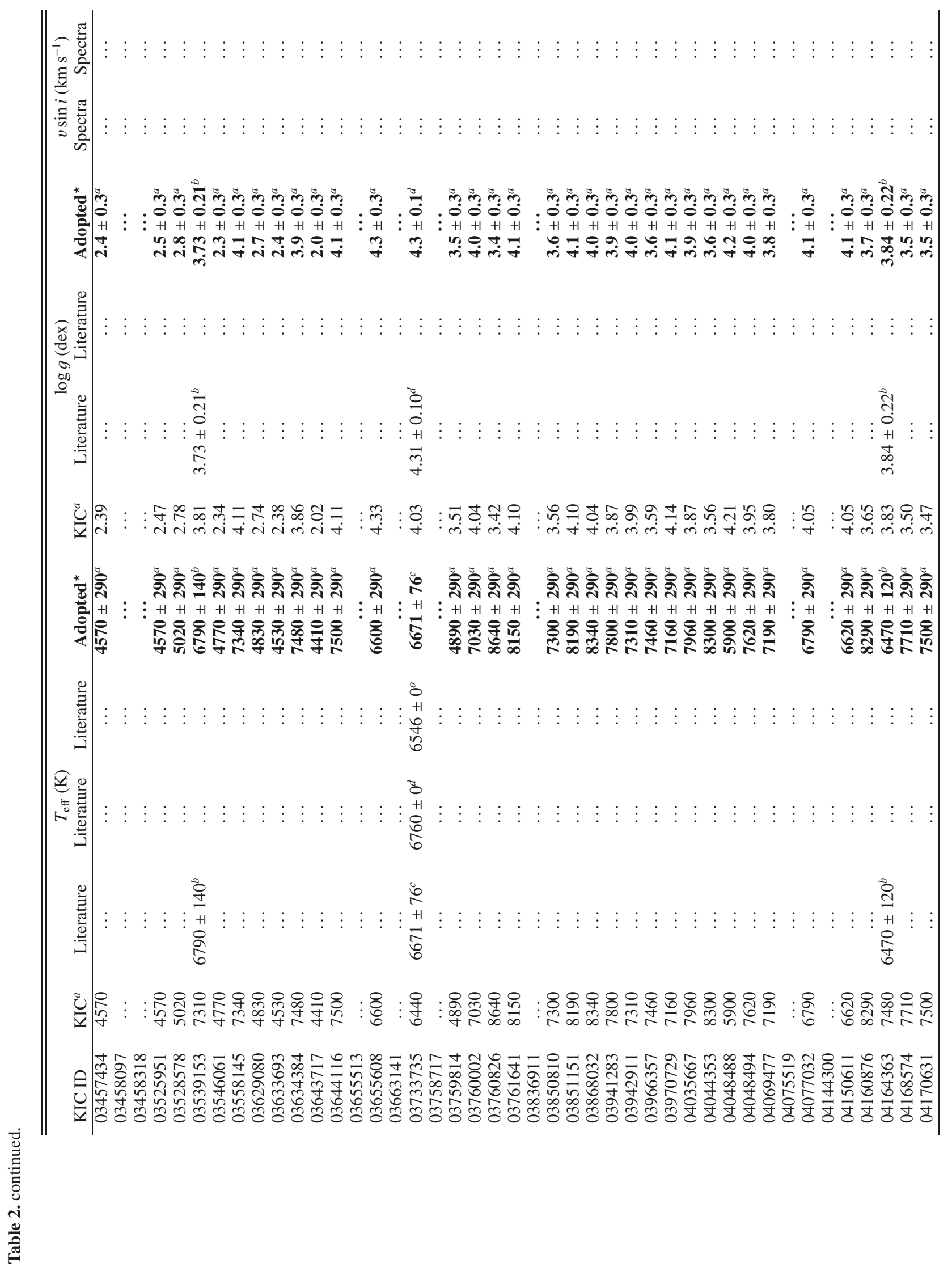




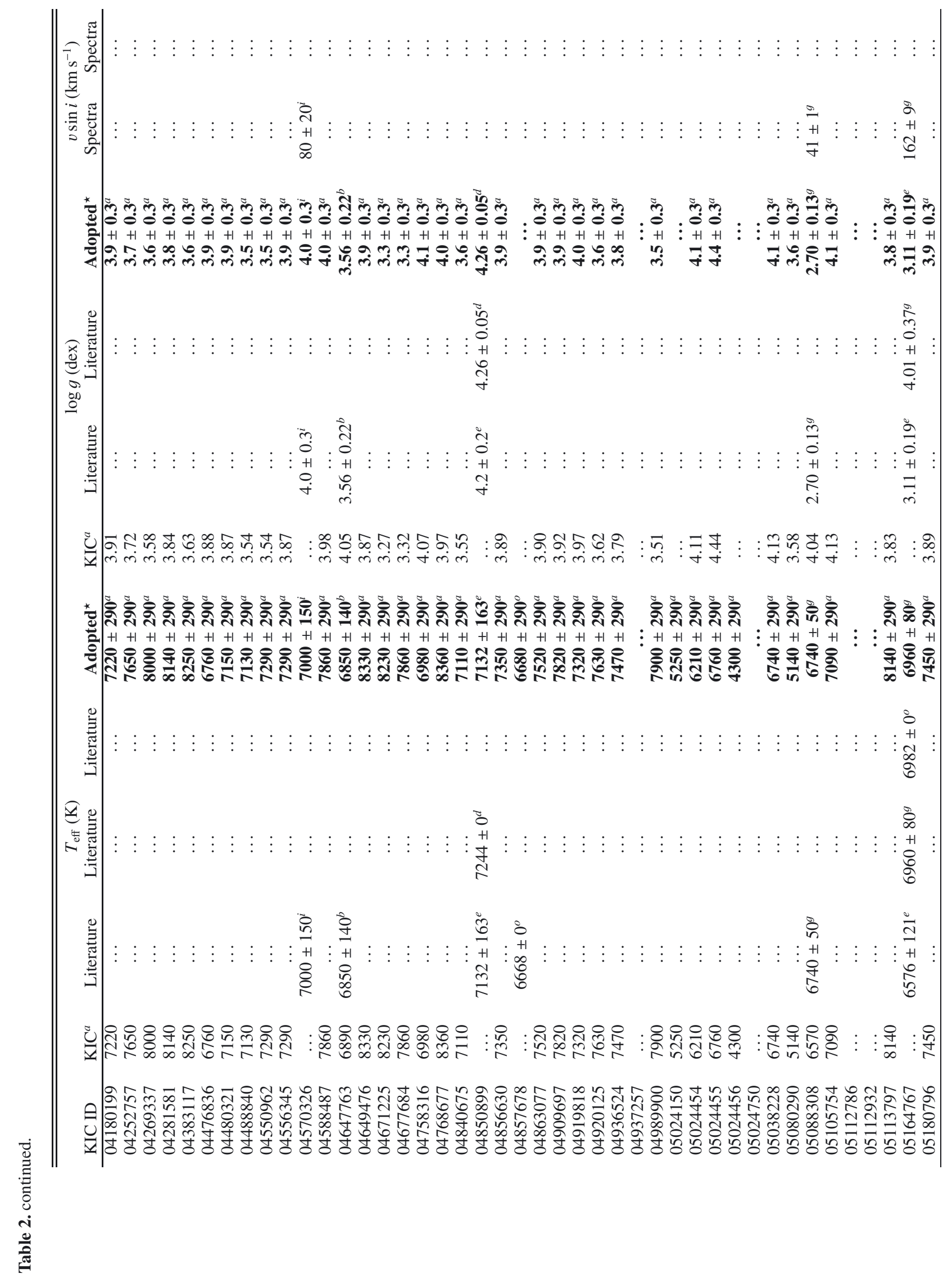


K. Uytterhoeven et al.: The Kepler characterization of the variability among A- and F-type stars. I.

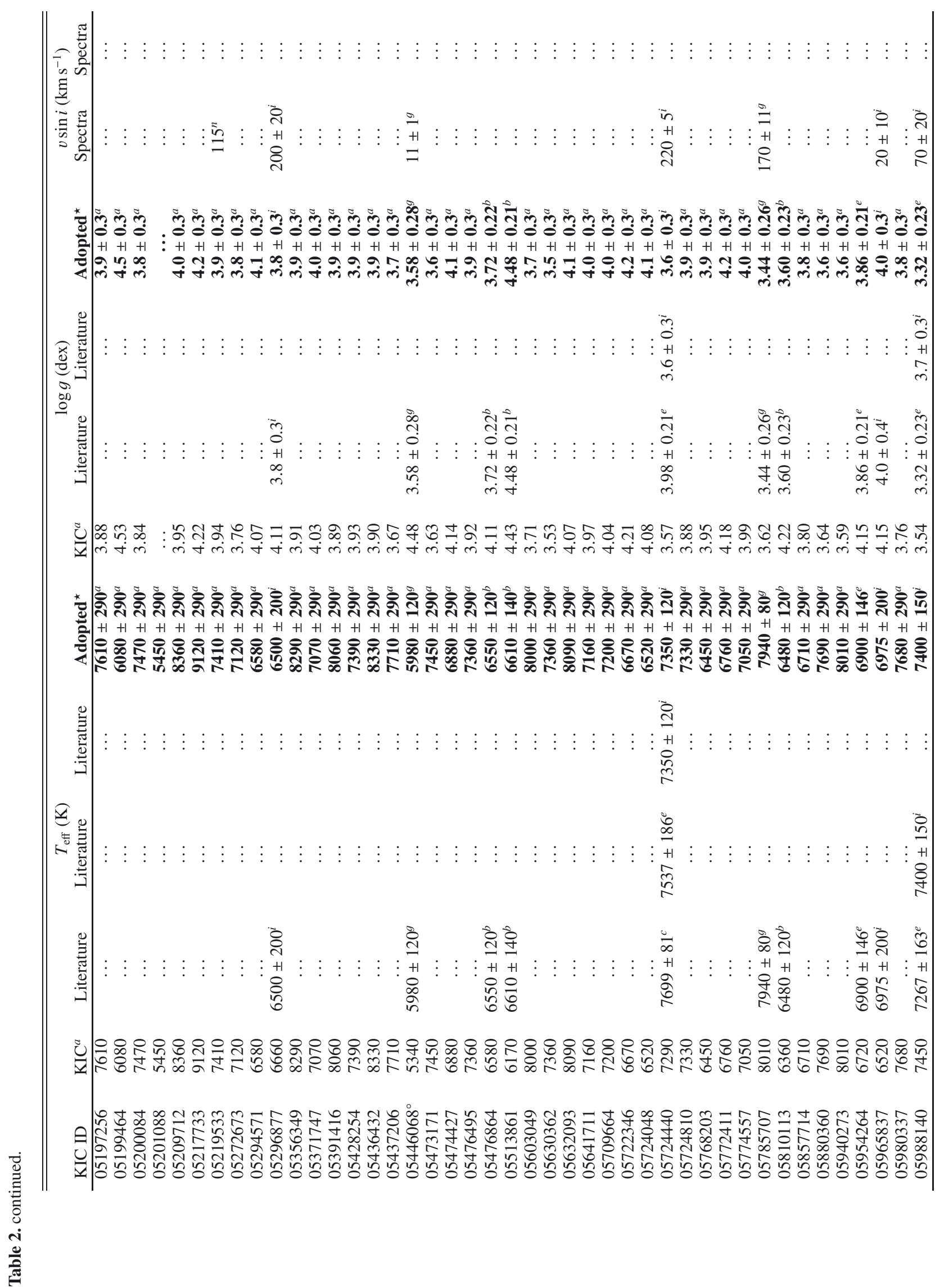

A125, page 43 of 70 


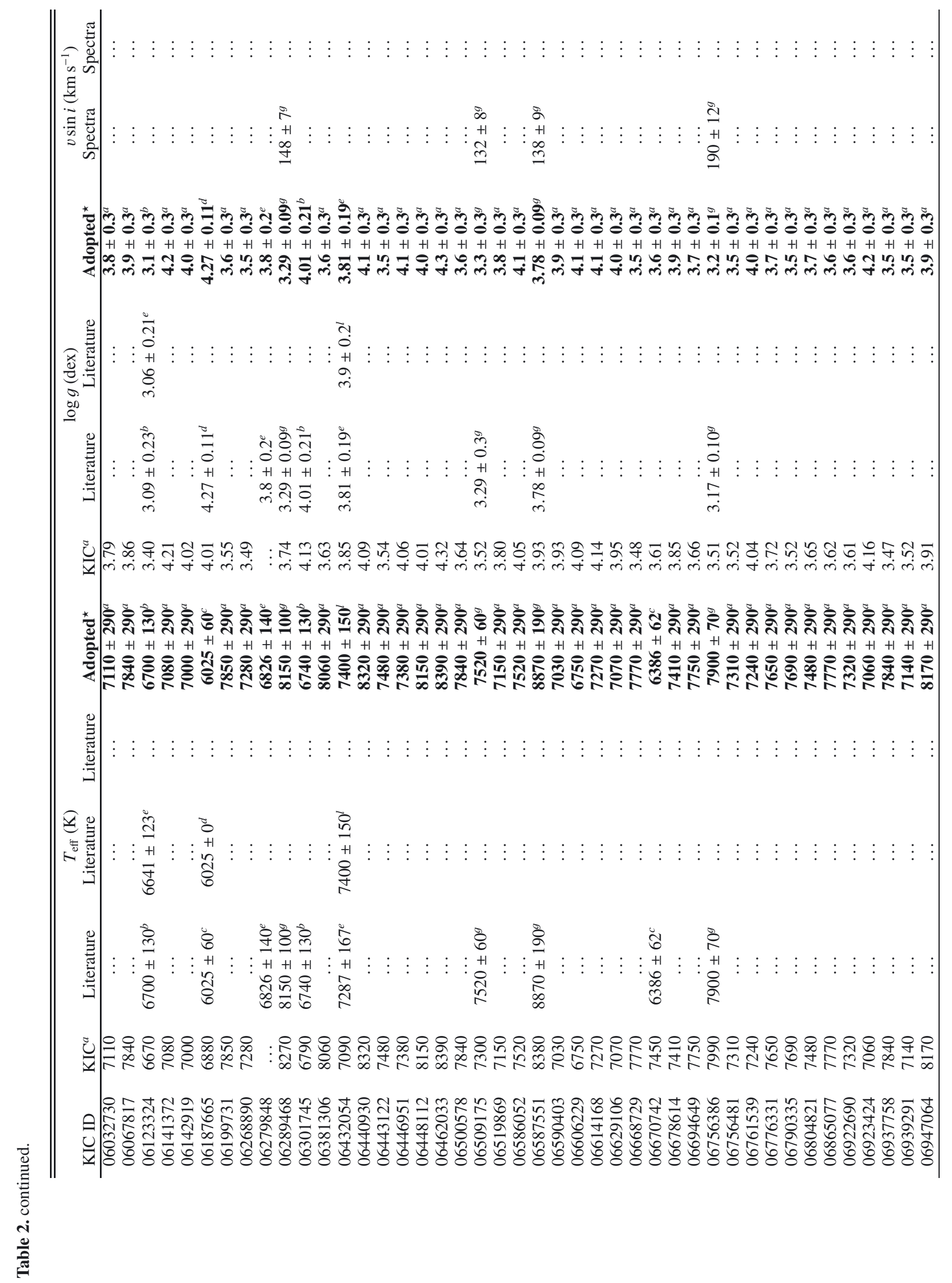


K. Uytterhoeven et al.: The Kepler characterization of the variability among A- and F-type stars. I.

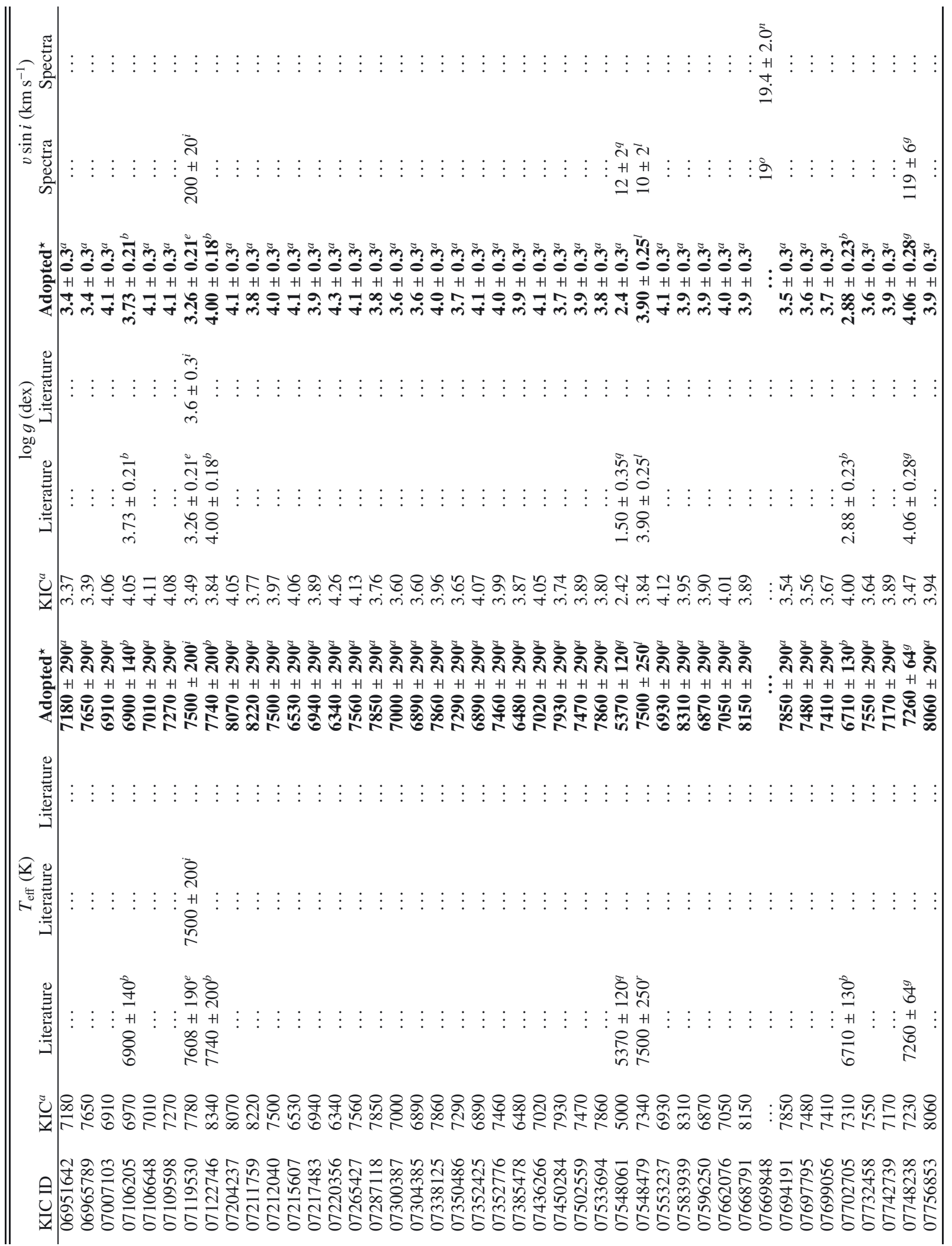




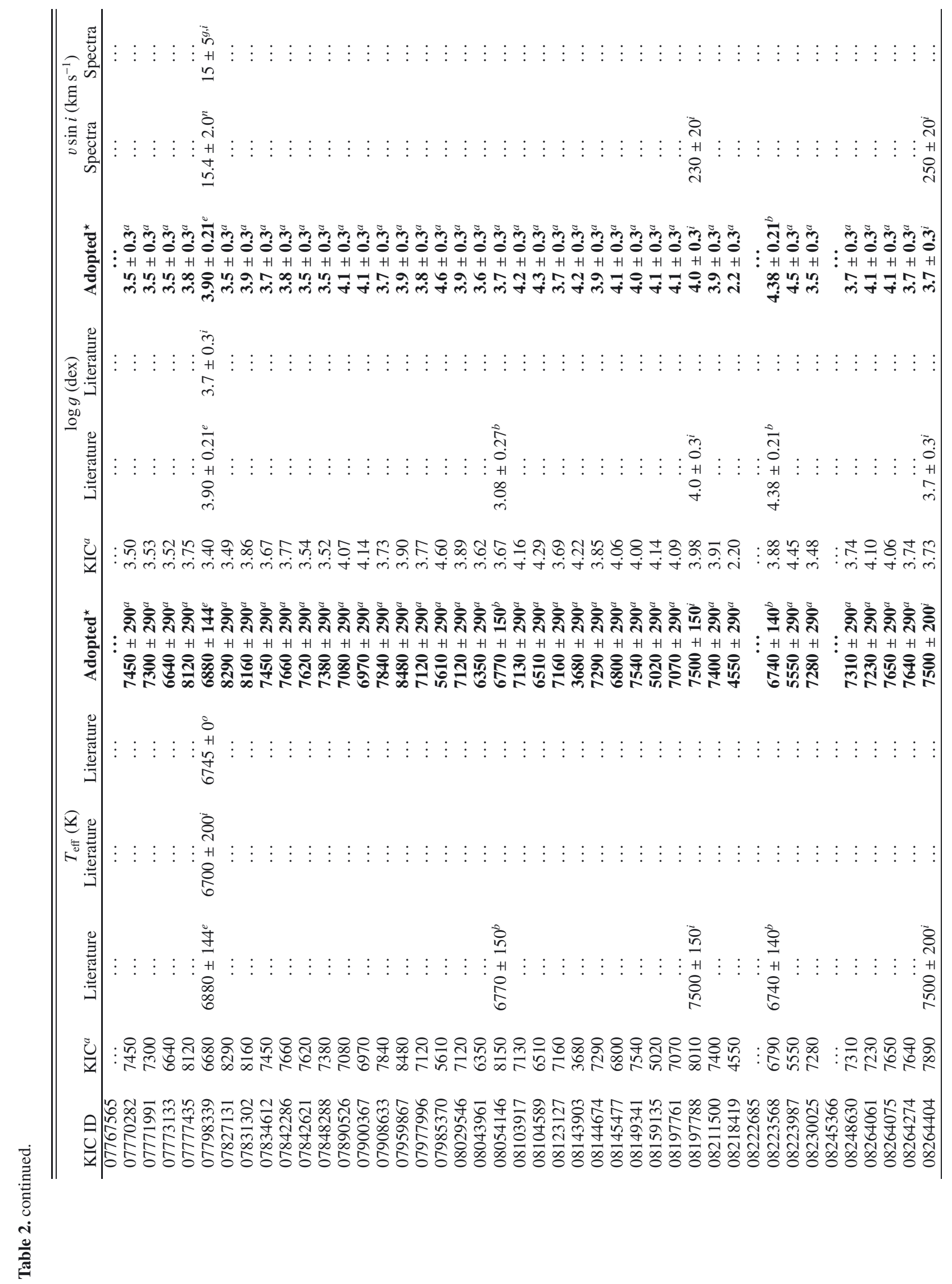


K. Uytterhoeven et al.: The Kepler characterization of the variability among A- and F-type stars. I.

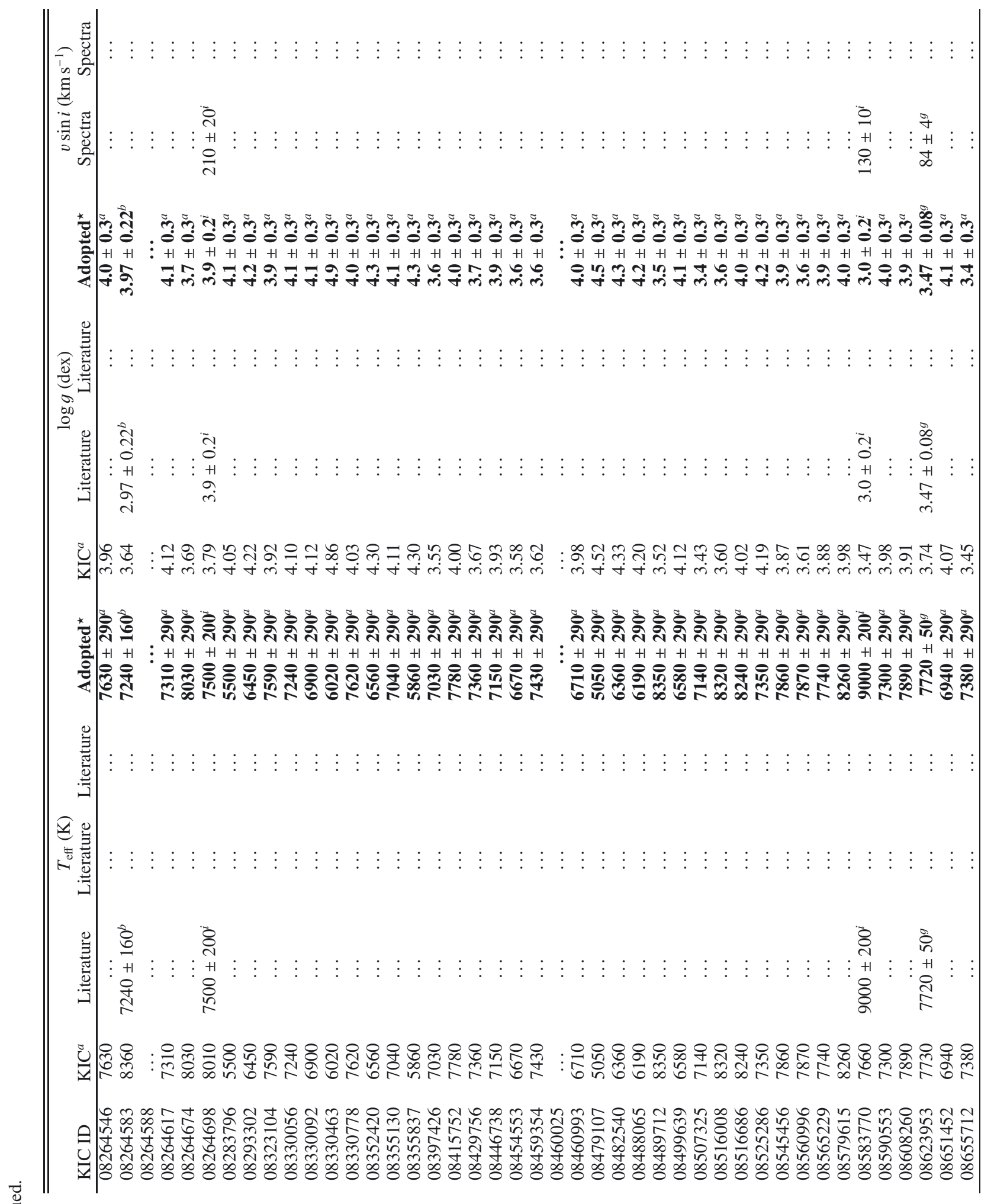




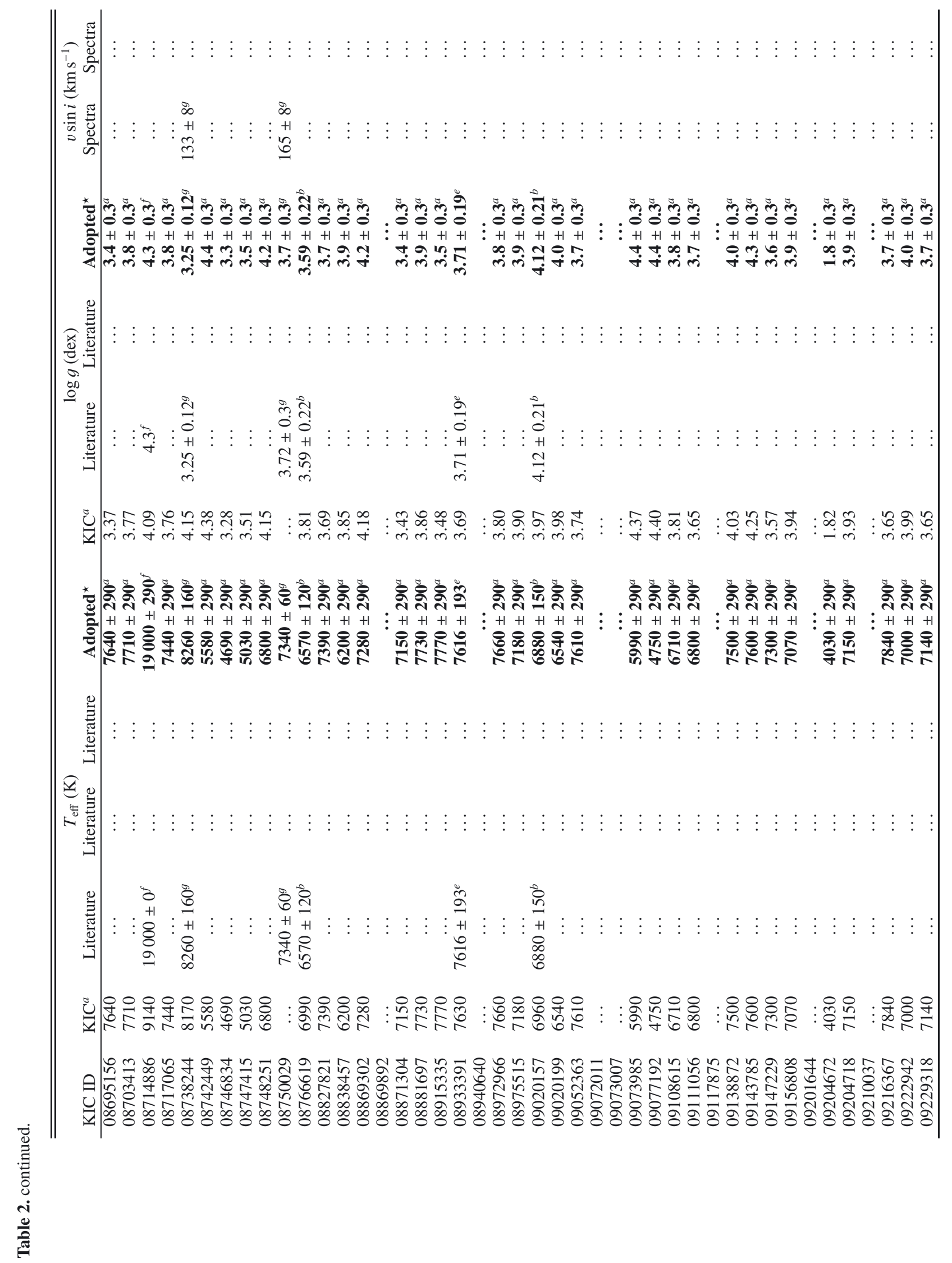


K. Uytterhoeven et al.: The Kepler characterization of the variability among A- and F-type stars. I.

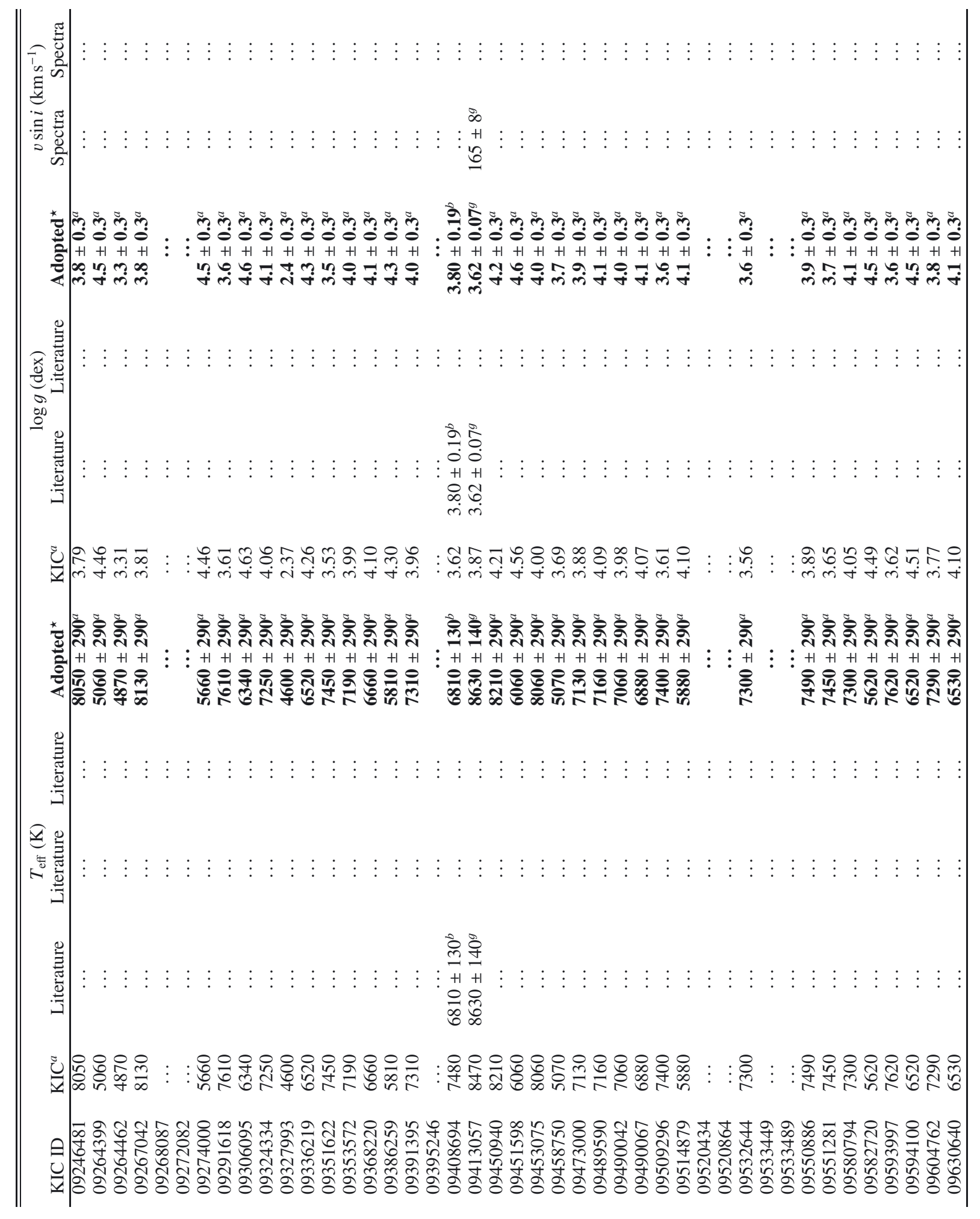




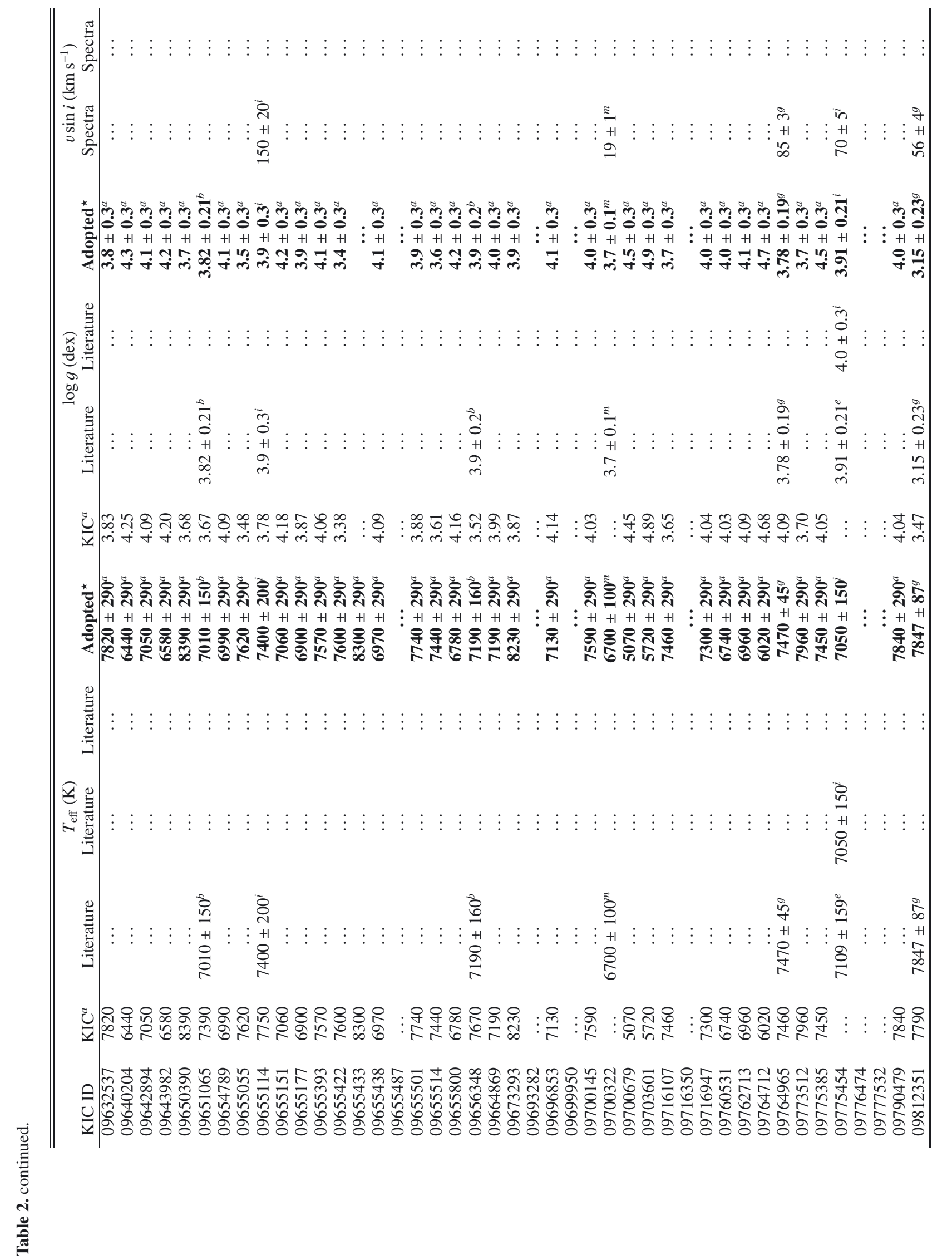


K. Uytterhoeven et al.: The Kepler characterization of the variability among A- and F-type stars. I.

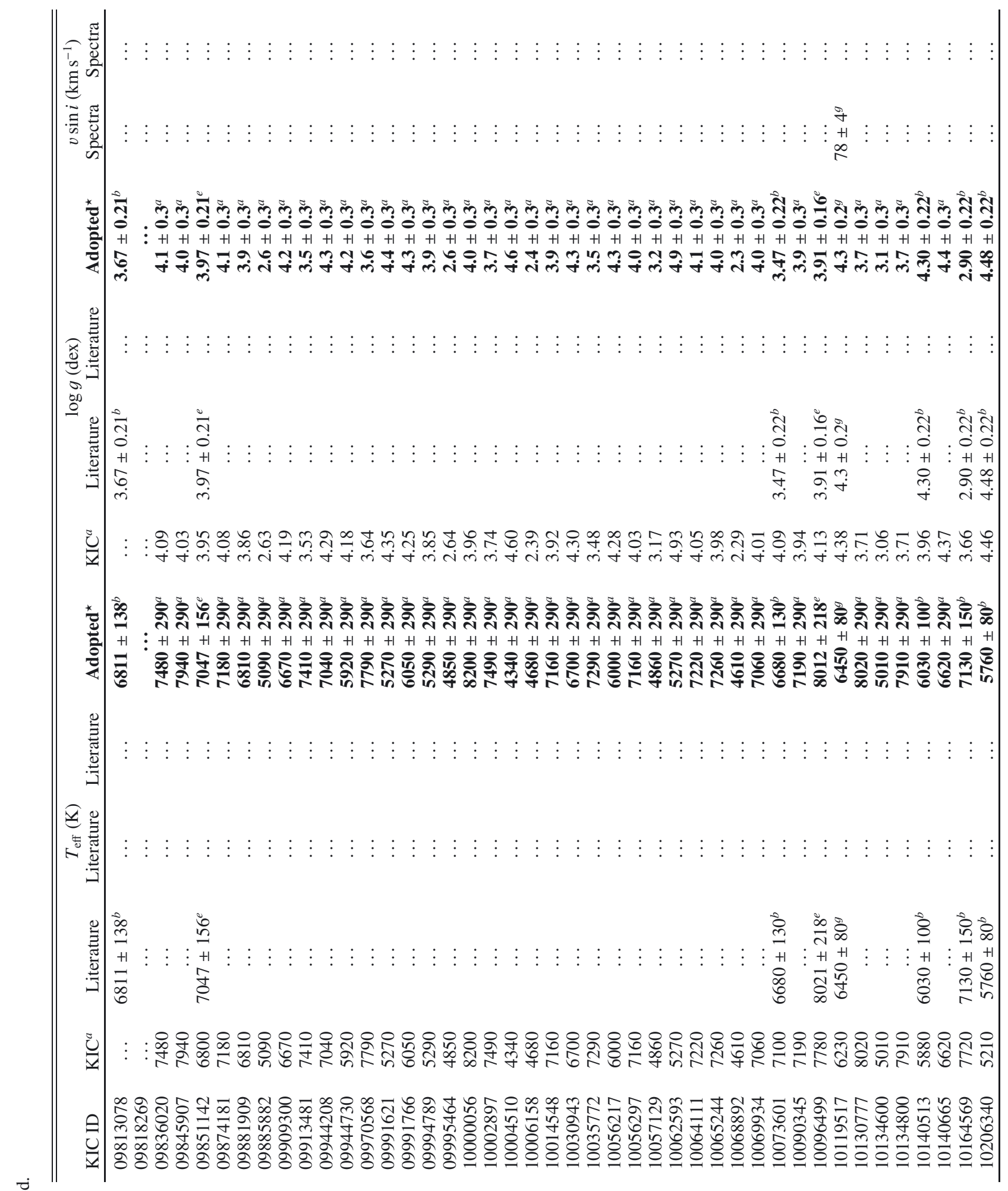




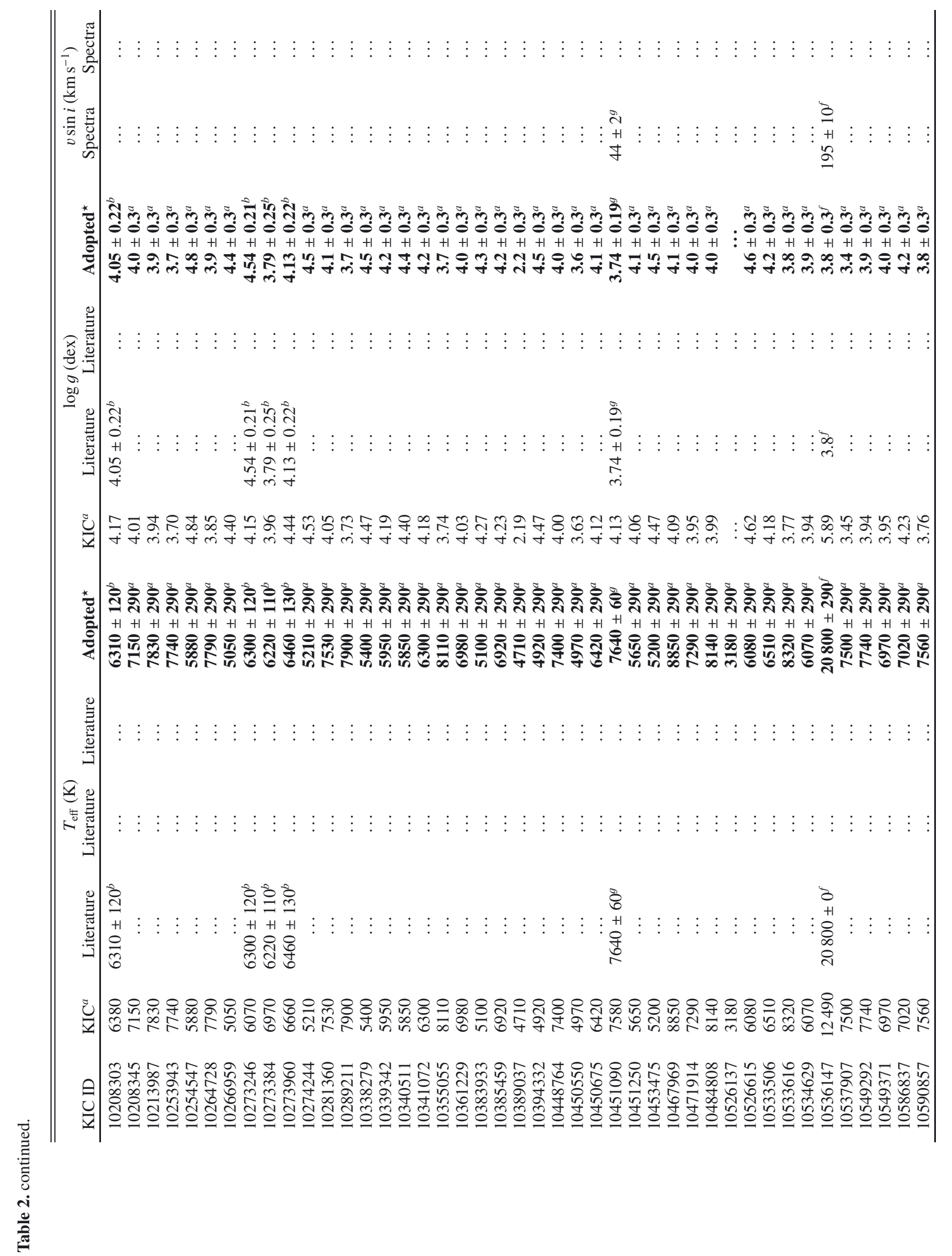


K. Uytterhoeven et al.: The Kepler characterization of the variability among A- and F-type stars. I.

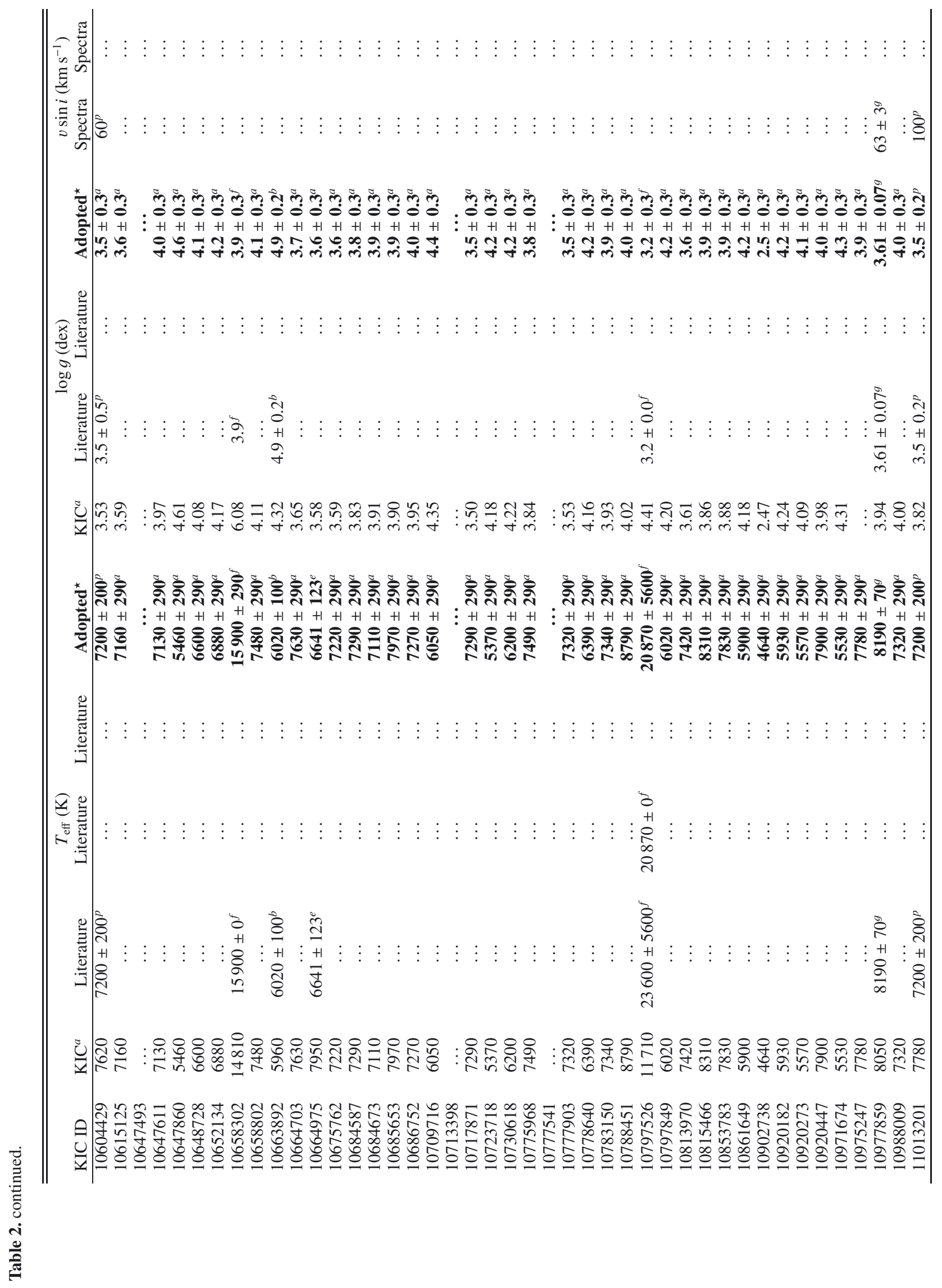




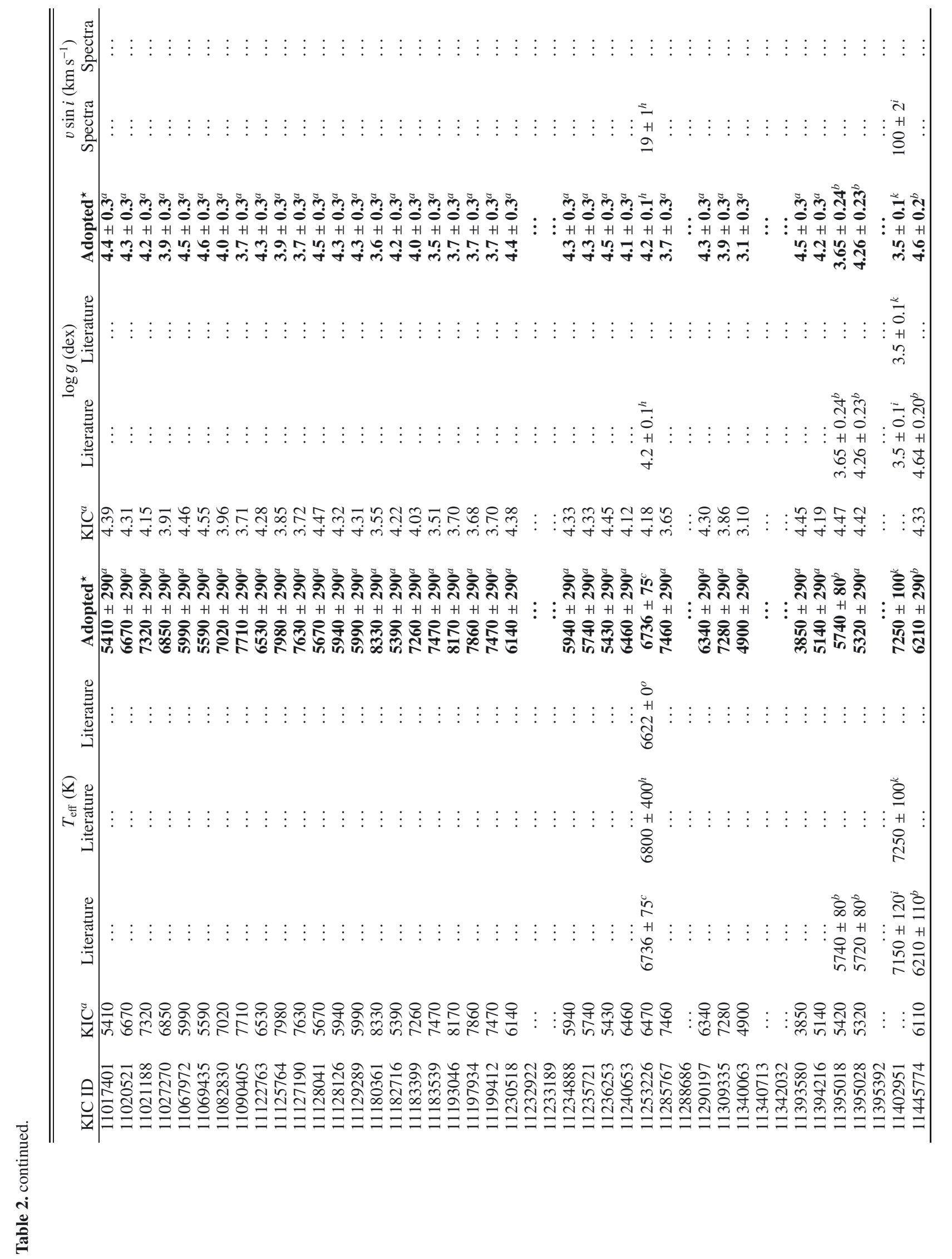


K. Uytterhoeven et al.: The Kepler characterization of the variability among A- and F-type stars. I.

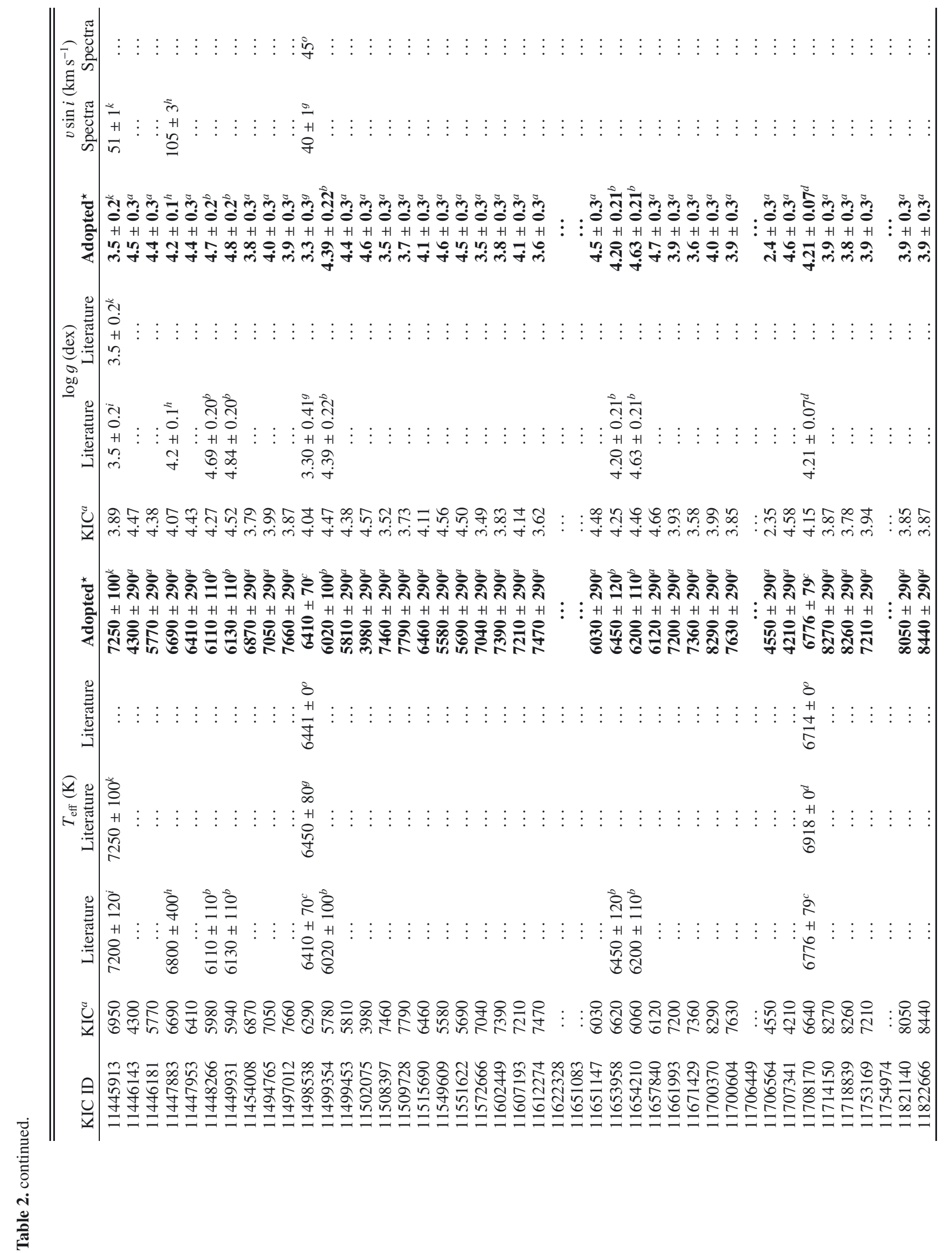

A125, page 55 of 70 


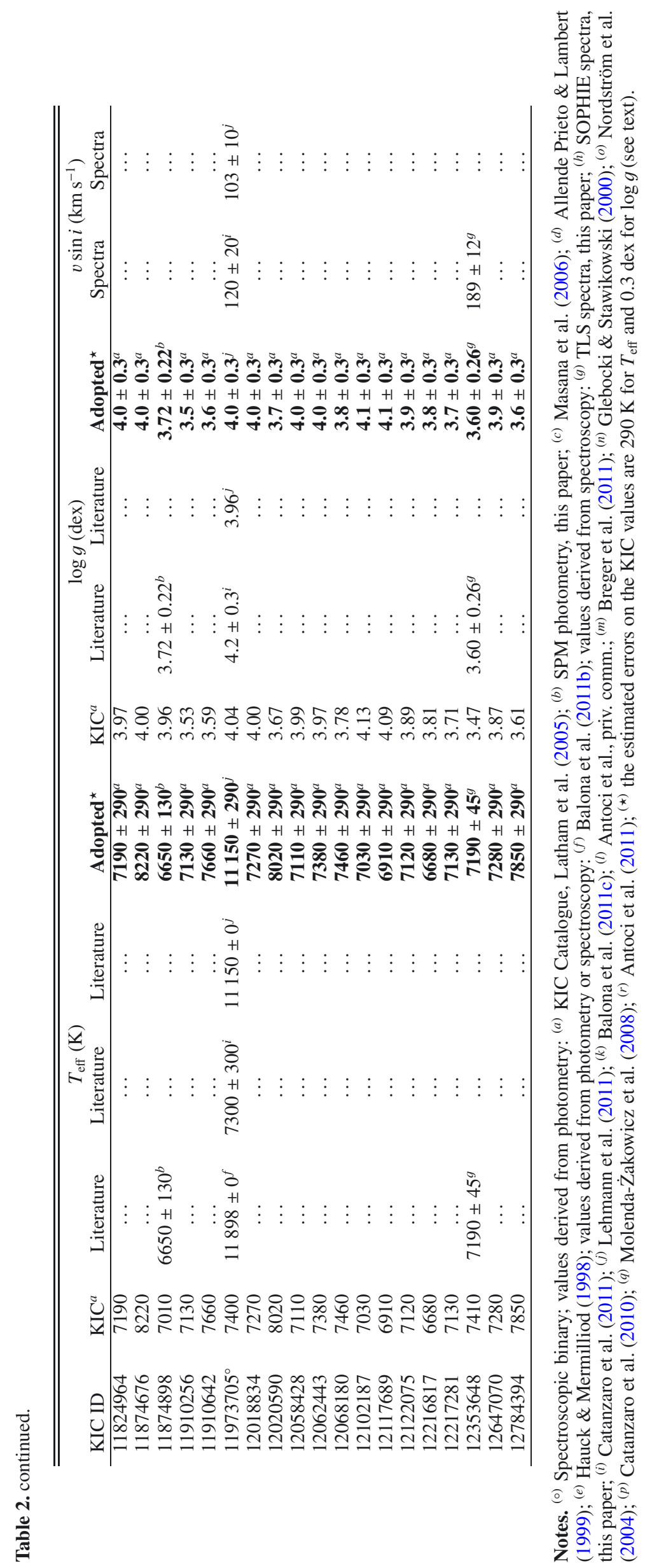


K. Uytterhoeven et al.: The Kepler characterization of the variability among A- and F-type stars. I.

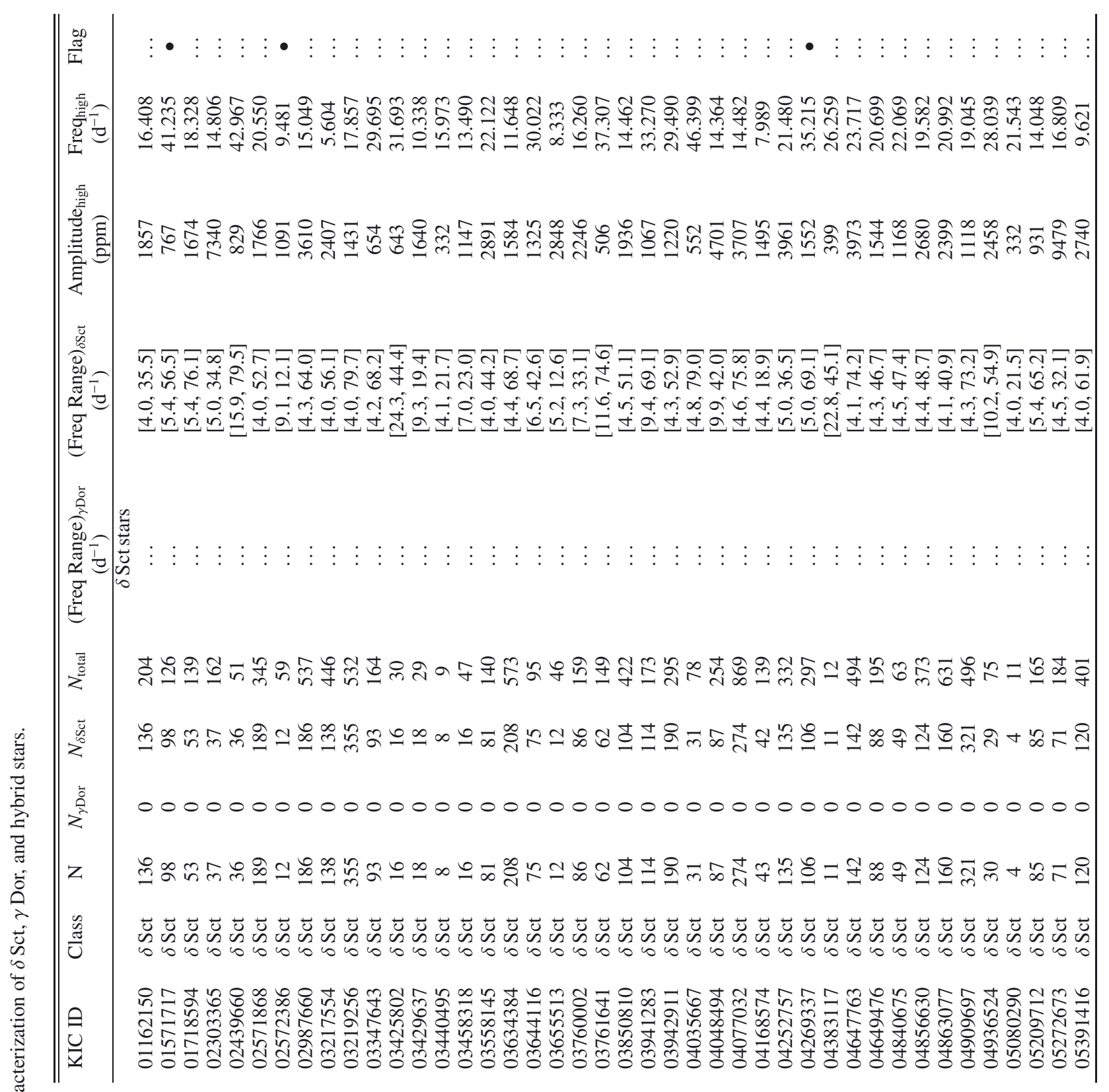




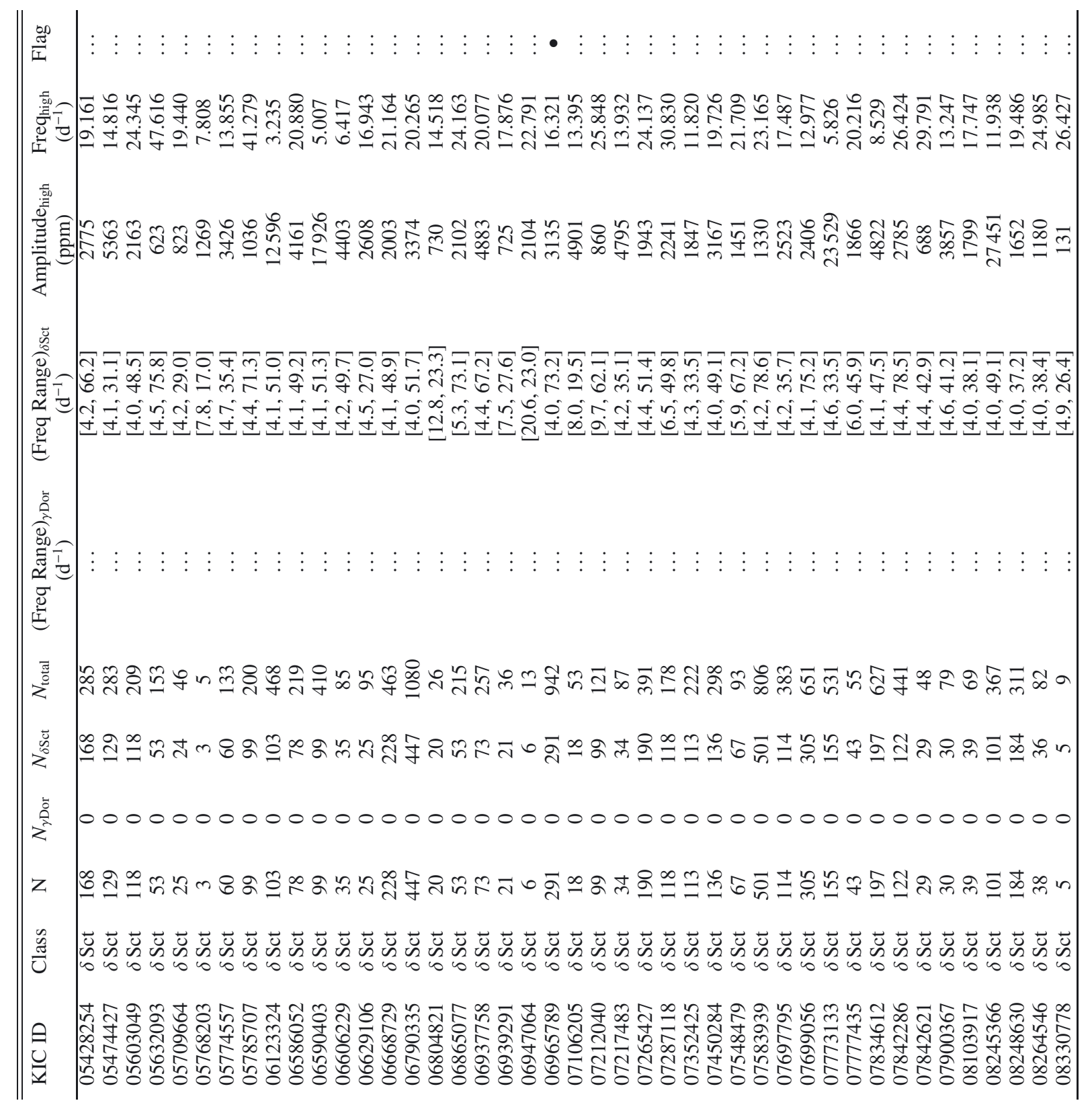




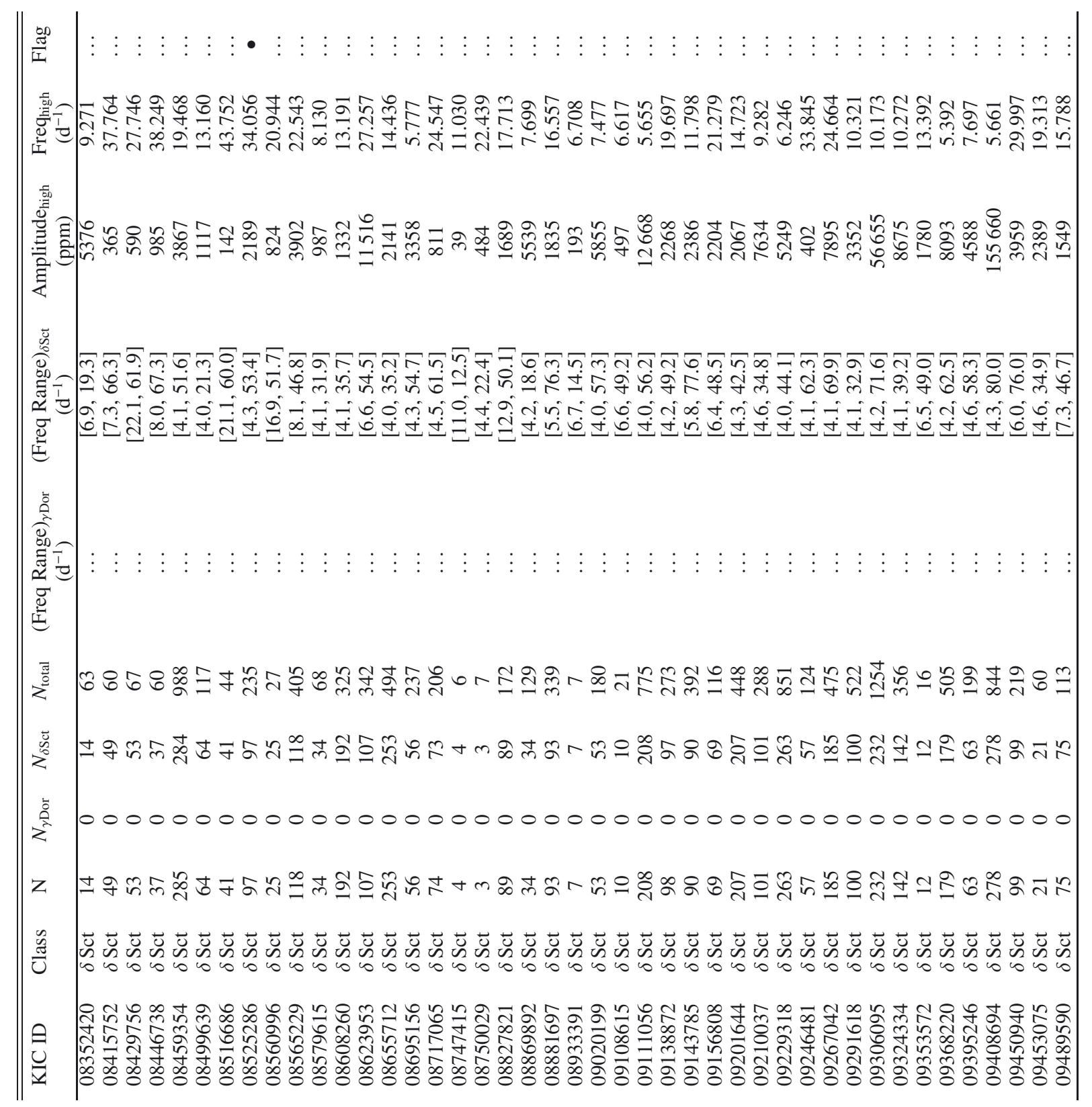



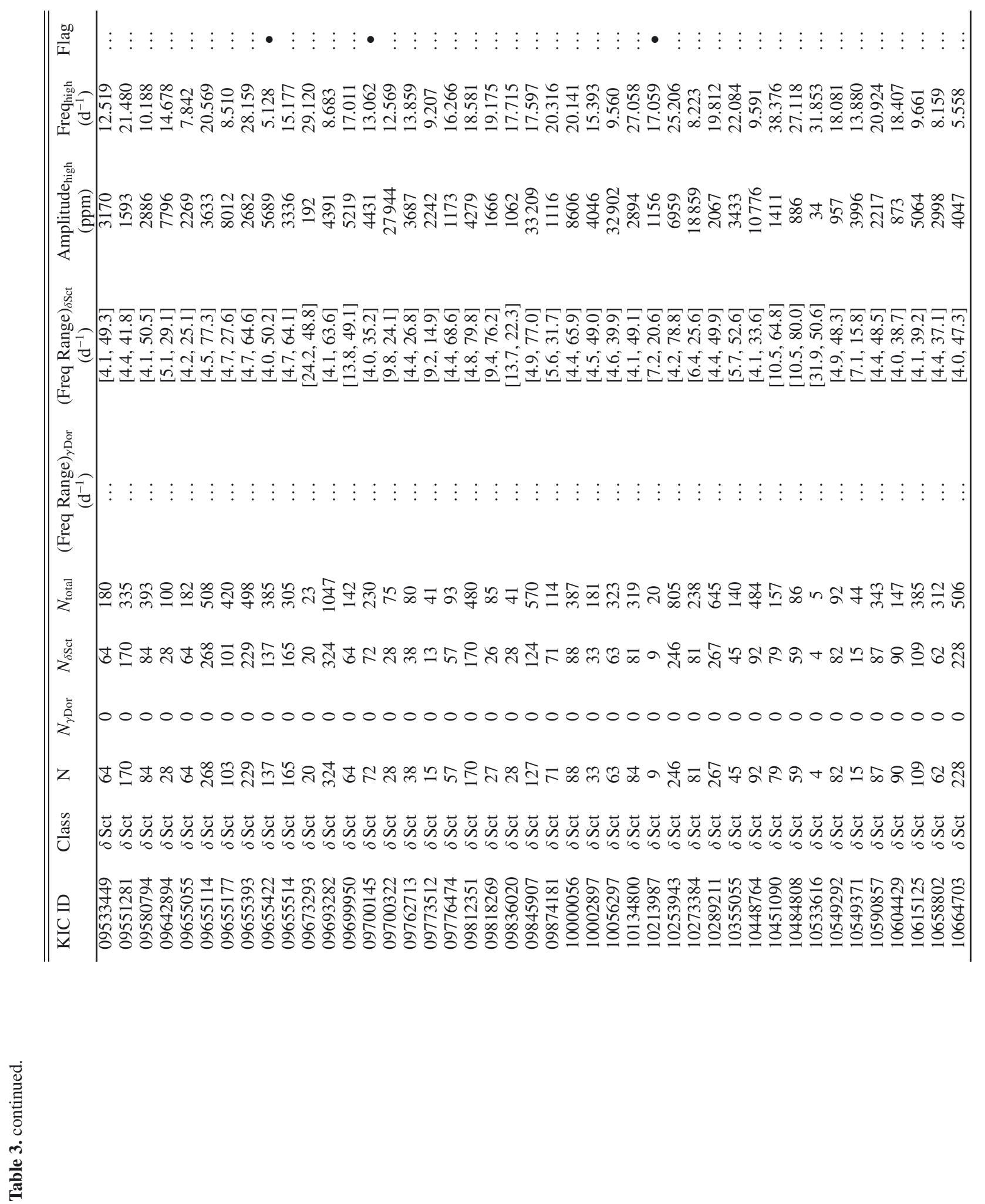


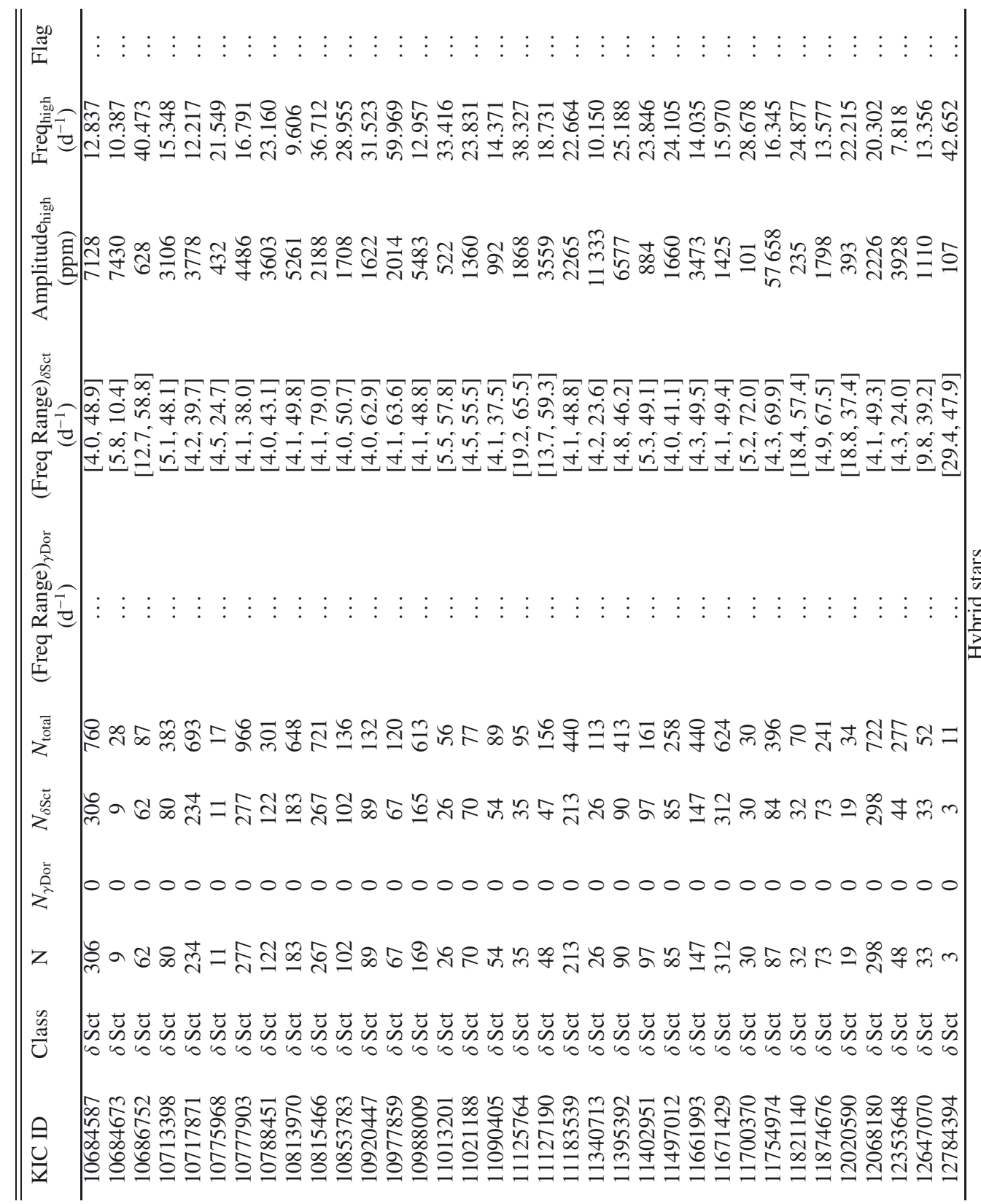

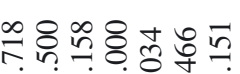

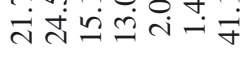

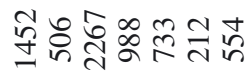
$\exists \sigma=750$ 守的的过 nit-onio 的的的政

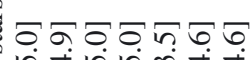
a 으응ㅇㅇㅇㅡ.

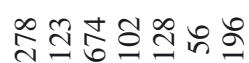

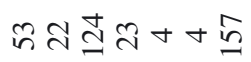
ลํำ

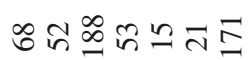
뭄 뭄 뭄 뭄 뭄

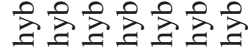

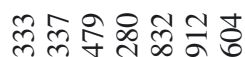
क节

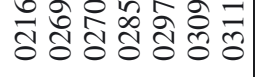




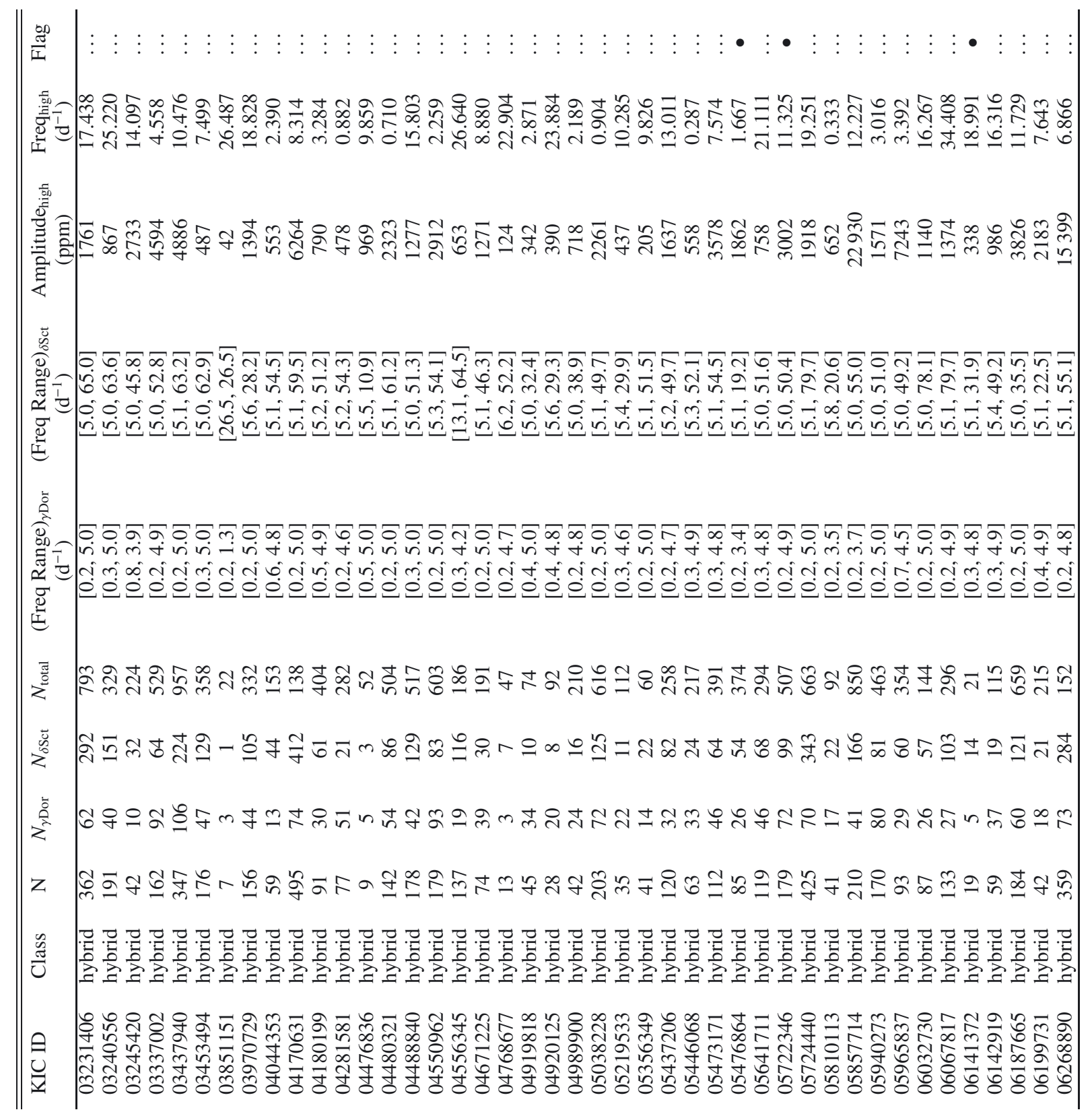


K. Uytterhoeven et al.: The Kepler characterization of the variability among A- and F-type stars. I.

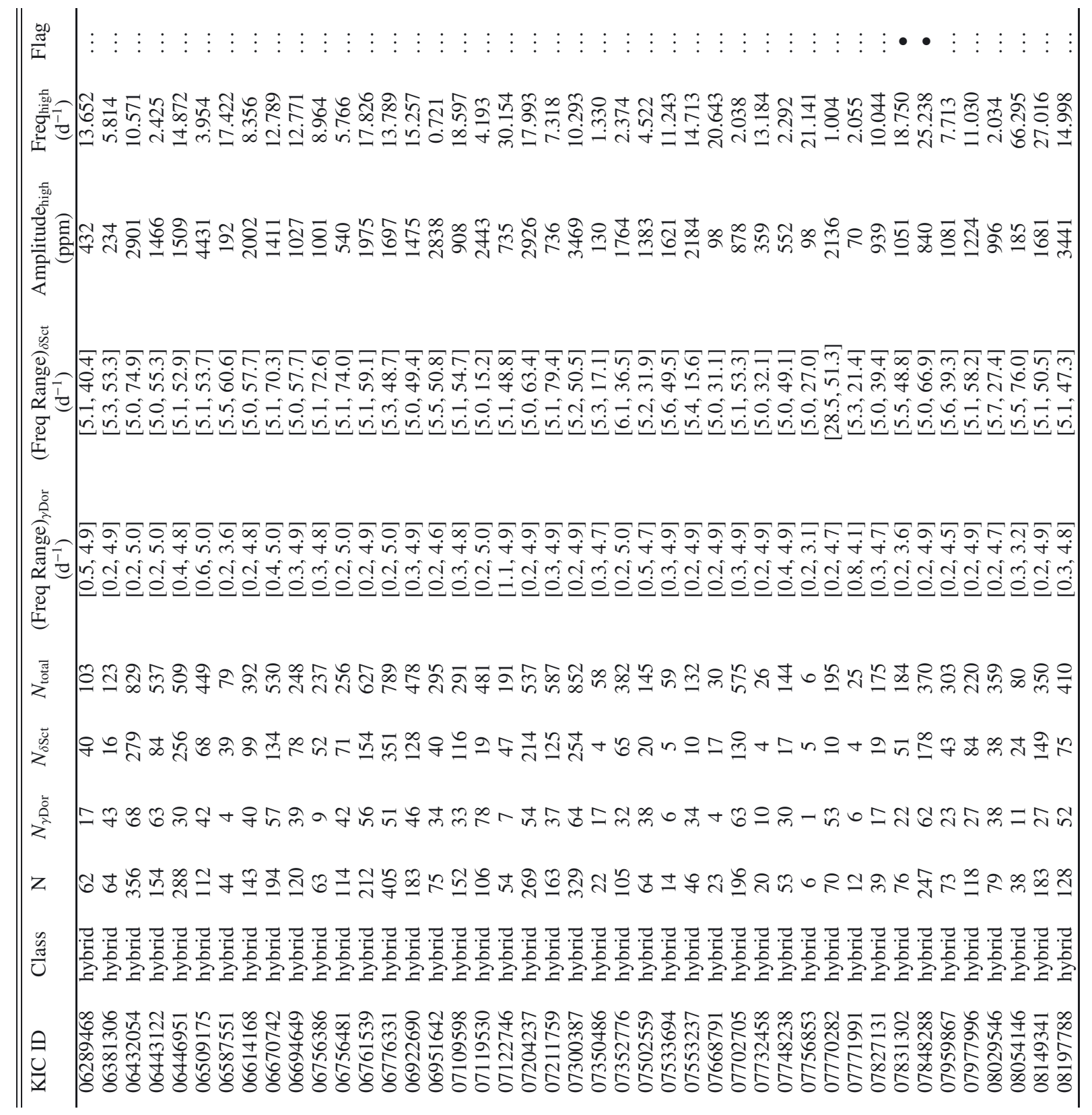



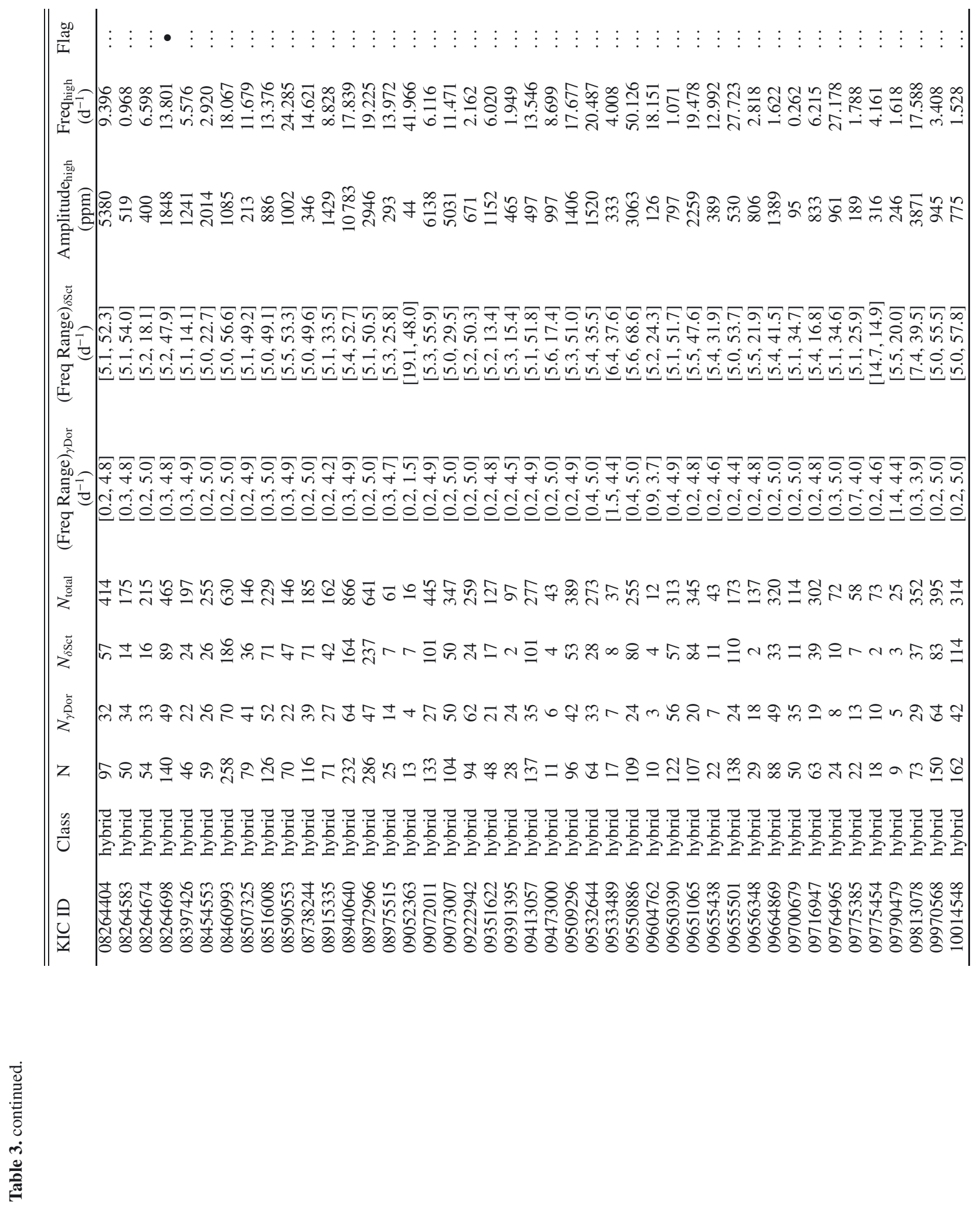
K. Uytterhoeven et al.: The Kepler characterization of the variability among A- and F-type stars. I.
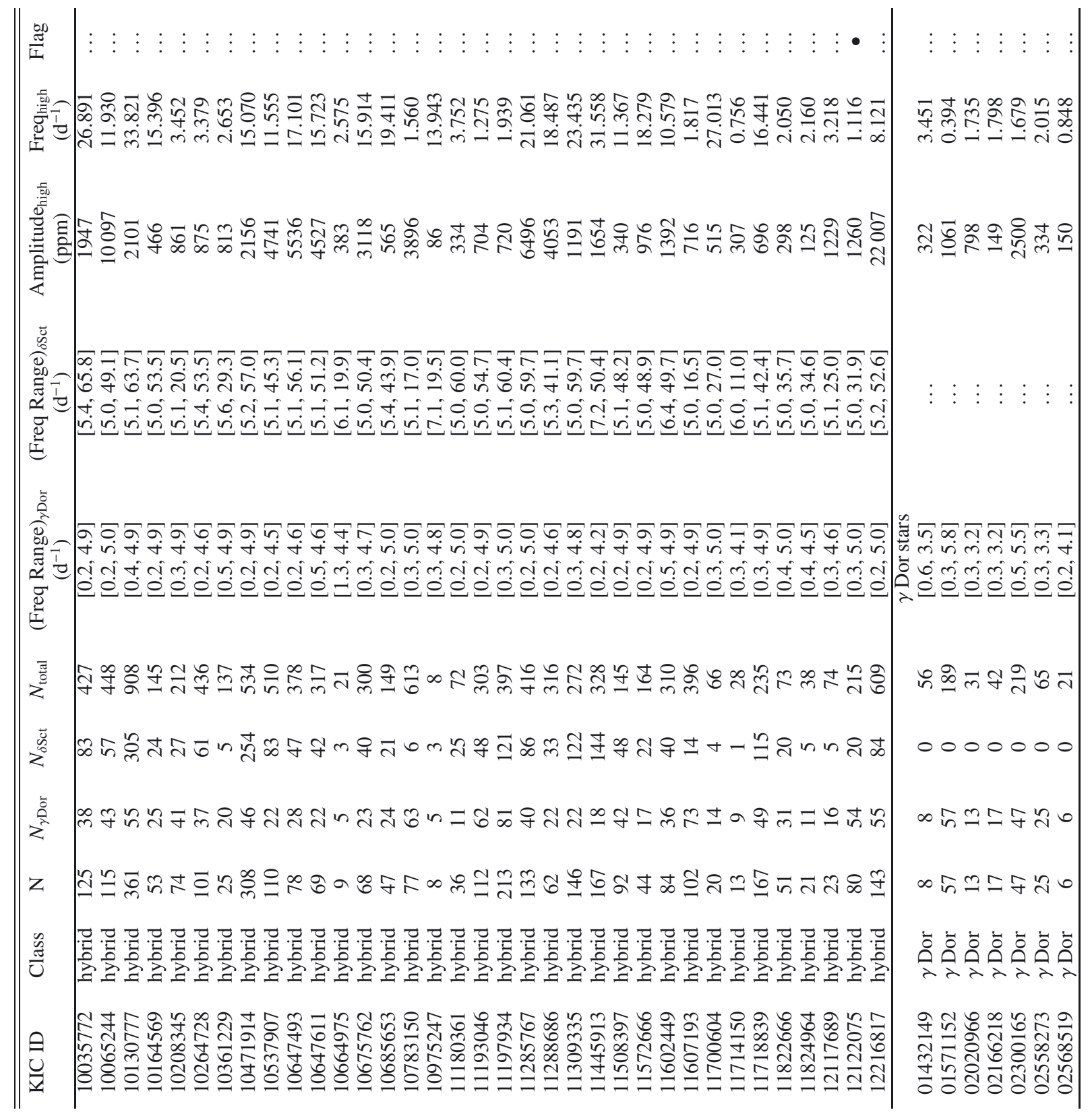

भल लिर 00

mo- - तo

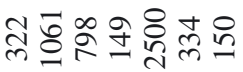

Бळ ठิm 으응ㅇㅇ

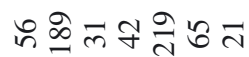
0000000

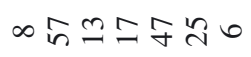

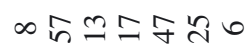

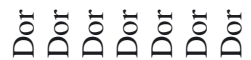
入入入入入入 守 तె

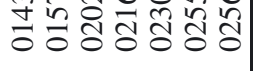




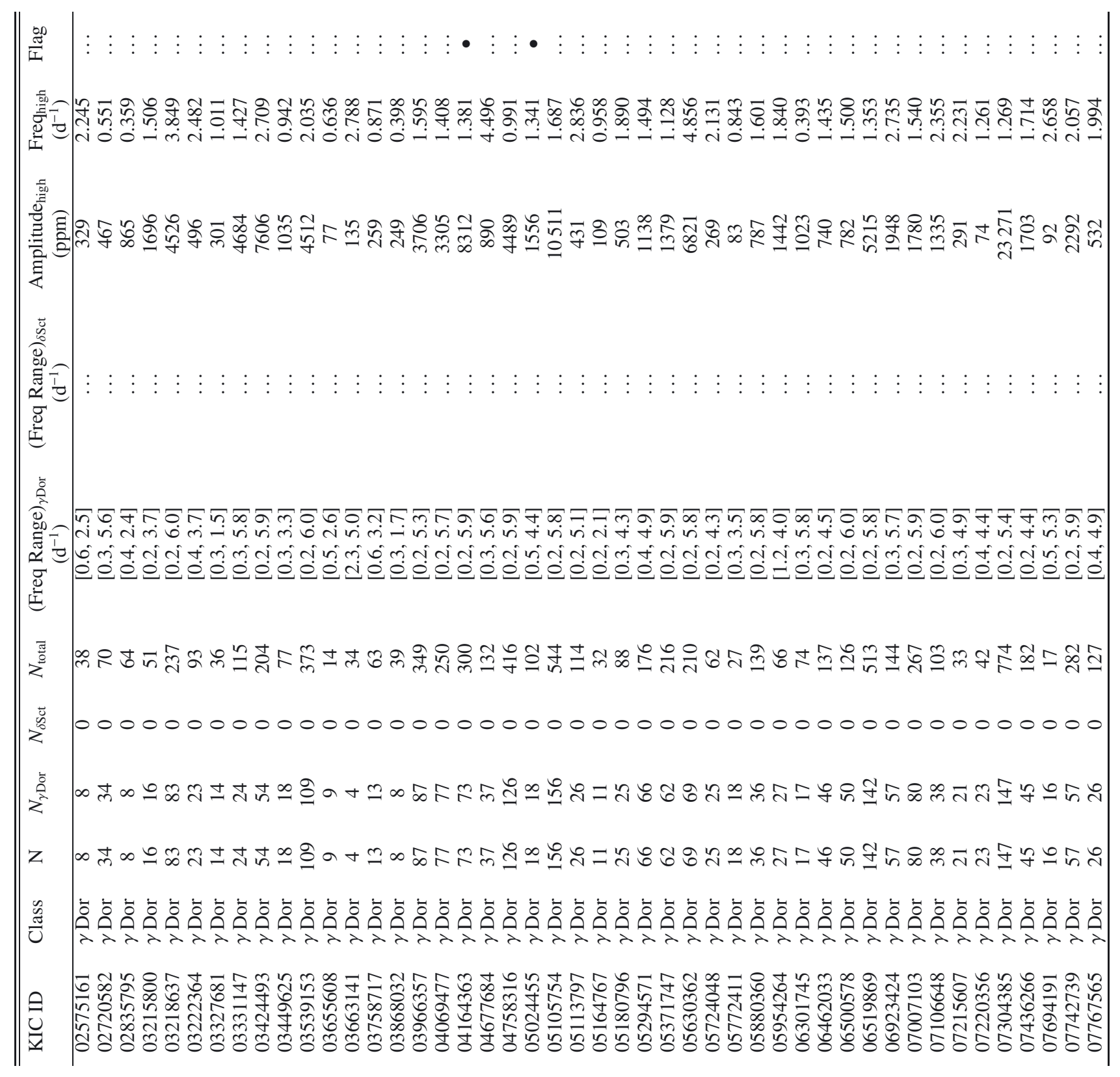




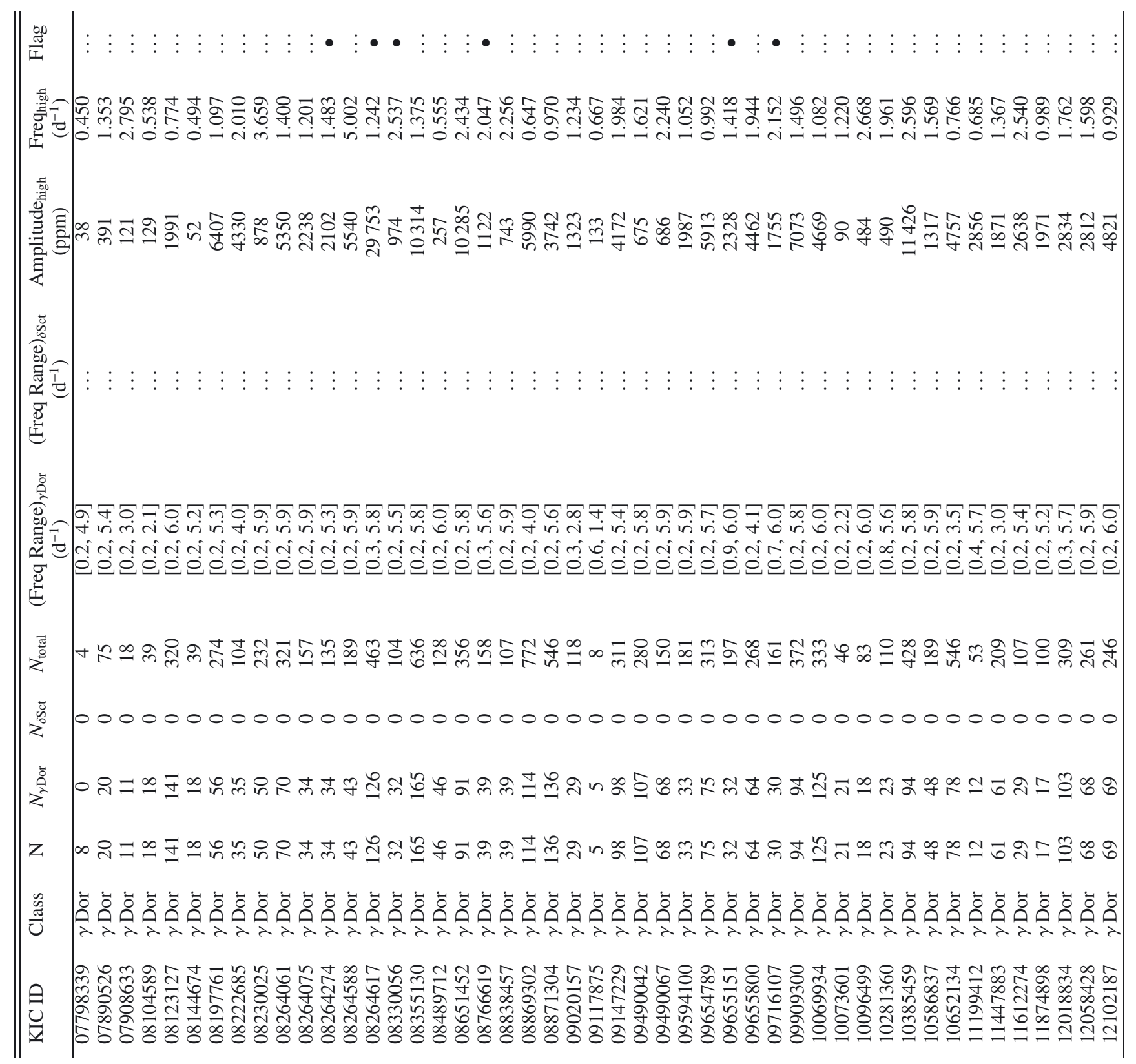




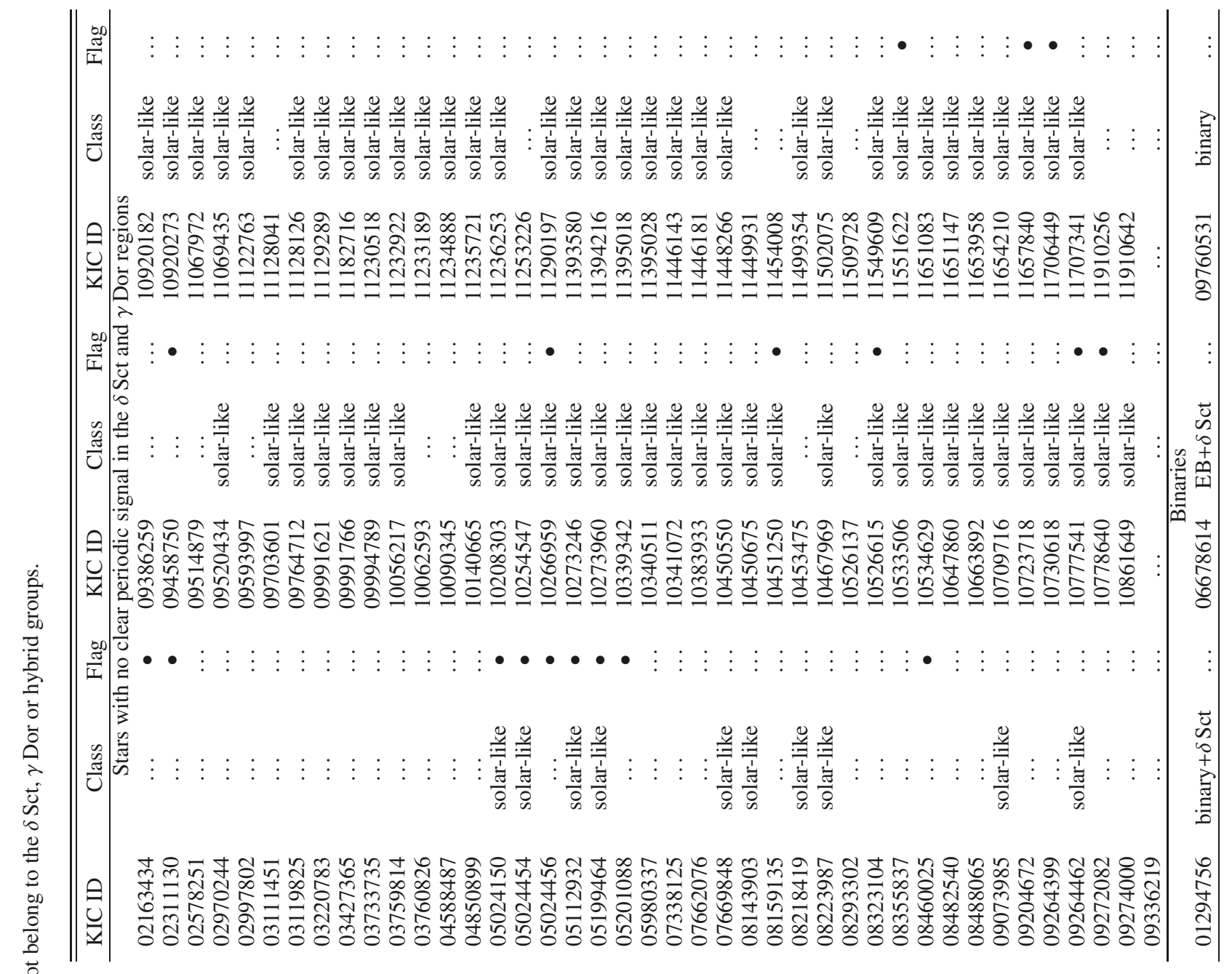




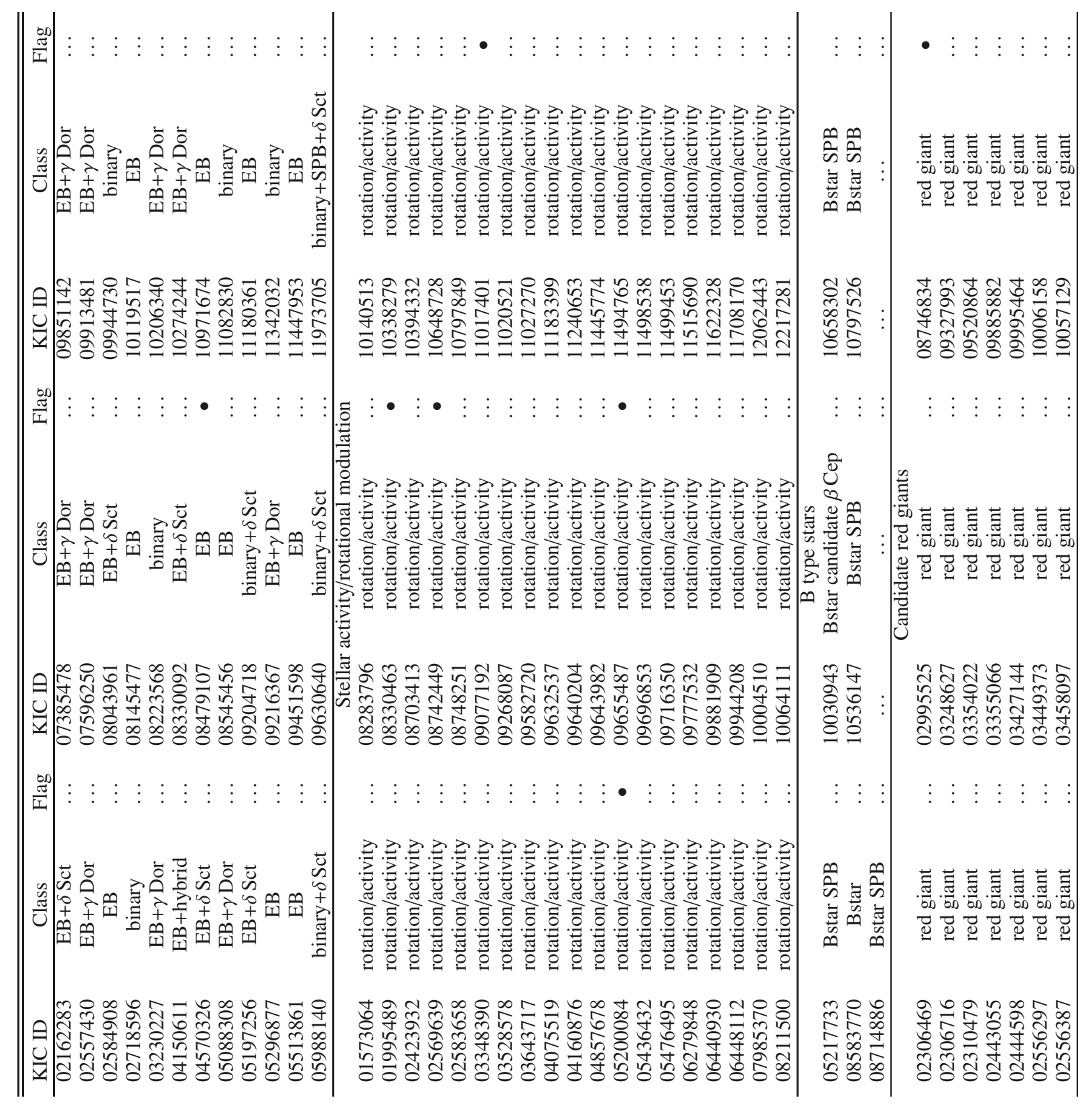




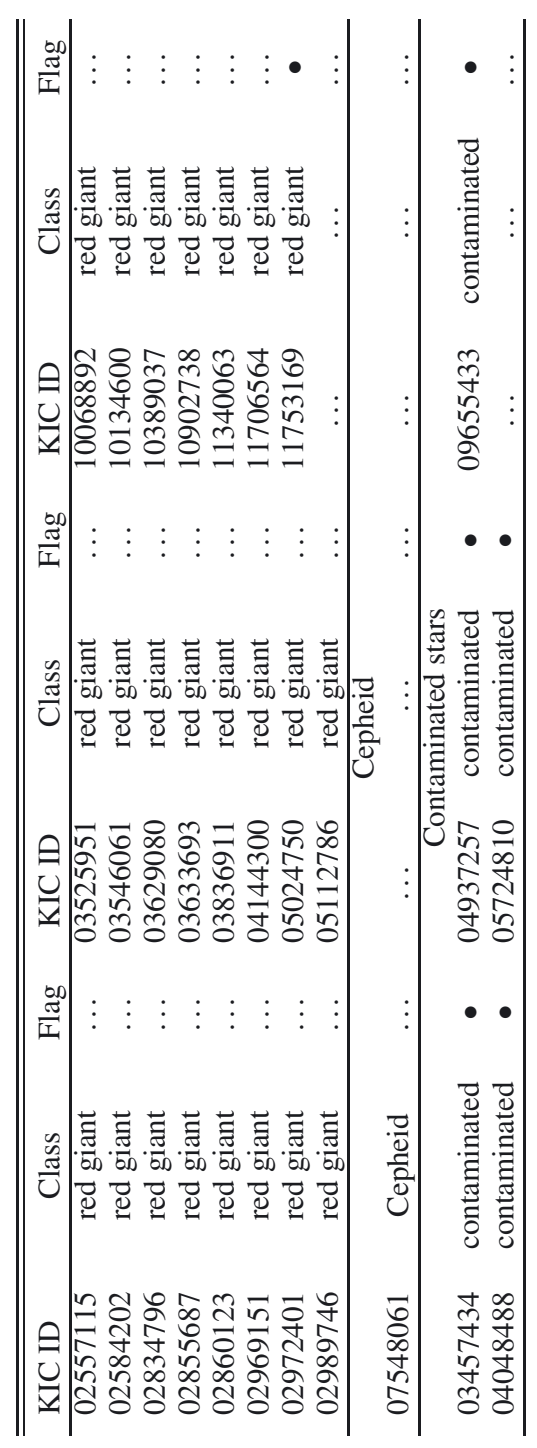

